\title{
Transformações em modelos de séries temporais
}

\author{
Amanda dos Santos Gomes
}

TESE APRESENTADA

$\mathrm{AO}$

Instituto de MatemáticA E EstatísticA

DA

UNIVERSIDADE DE SÃO PAULO

PARA

OBTENÇÃO DO TÍTULO

$\mathrm{DE}$

DOUTOR EM CIÊNCIAS

Programa: Estatística

Orientador: Prof. Dr. Pedro Alberto Morettin

Durante o desenvolvimento deste trabalho a autora recebeu auxílio financeiro da $\mathrm{CAPES} / \mathrm{CNPq}$

São Paulo, julho de 2012 


\section{Transformações em modelos de séries temporais}

Esta versão definitiva da tese contém as correções e alterações sugeridas pela Comissão Julgadora durante a defesa realizada por Amanda dos Santos Gomes.

Banca Examinadora:

- Prof. Dr. Pedro Alberto Morettin (orientador) - IME-USP.

- Profa. Dra. Clélia Maria de Castro Toloi - IME-USP.

- Prof. Dr. Luiz K. Hotta - IMECC-UNICAMP.

- Prof. Dr. Gauss M. Cordeiro - CCEN-UFPE.

- Profa. Dra. Clarice B. Demétrio - ESALQ-USP. 


\section{Agradecimentos}

- A Deus, por sempre me guiar.

- Aos meus pais, Vicente e Avany que com amor, carinho e dedicação me educaram e me deram tudo que é necessário para ser feliz.

- À minha filha, Natália, por sempre me dar forças para lutar em todos os momentos.

- Ao meu orientador, Pedro A. Morettin, pelos ensinamentos, segurança, paciência e incentivo.

- Ao professor doutor Gauss M. Cordeiro, pelas ideias.

- Ao professor doutor Francisco J. A. Cysneiros, pelos seus esclarecimentos em alguns artigos científicos.

- À Unidade Acadêmica de Matemática e Estatística da UFCG, pela contribuição na minha formação acadêmica e por unirem esforços para a minha liberação.

- Às minhas amigas, Luz Marina, Nubia e Débora por serem minhas amigas.

- Aos meus incontáveis colegas do IME-USP, pelas horas enriquecedoras de estudo e diversão.

- À CAPES e CNPq, pelo apoio financeiro.

- Aos participantes da banca examinadora, pela presença e sugestões.

- A todos que de alguma forma contribuiram para a realização deste trabalho. 



\section{Resumo}

\section{Transformações em Modelos de Séries Temporais}

Cordeiro e Andrade (2009) incorporam a ideia de variável resposta transformada ao modelo GARMA, autorregressivo e de médias móveis generalizado, introduzido por Benjamin et al. (2003), desenvolvendo assim, o modelo TGARMA, autorregressivo e de médias móveis generalizado transformado.

O objetivo do presente trabalho é desenvolver o modelo TGARMA introduzido por Cordeiro e Andrade (2009) para distribuições condicionais simétricas contínuas com e sem heteroscedasticidade e uma possível função não linear para os parâmetros de regressão. Ao longo desta tese derivamos um processo iterativo para estimar os parâmetros desses modelos por máxima verossimilhança. Nós produzimos uma fórmula simples para estimar o parâmetro que define a transformação da variável resposta para uma subclasse de modelos. Fornecemos os momentos para a variável dependente original. Para o modelo homoscedástico, discutimos inferência de alguns parâmetros, propomos uma análise de diagnóstico e a definição de um resíduo padronizado. Finalmente, para ilustrar a teoria desenvolvida, tanto no caso homoscedástico quanto no caso heteroscedástico, utilizamos conjuntos de dados reais e avaliamos os resultados desenvolvidos por meio de estudos de simulação.

Palavras-chave: distribuição simétrica, parâmetro de transformação, séries temporais. 



\section{Abstract}

\section{Transformations in Time Series Models}

Cordeiro and Andrade (2009) incorporate the idea of transforming the response variable to the GARMA model, generalized autoregressive moving average, introduced by Benjamin et al. (2003), thus developing the TGARMA model, transformed generalized autoregressive moving average.

The goal of this thesis is to develop the TGARMA model introduced by Cordeiro and Andrade (2009) for symmetric continuous conditional distributions and a possible non-linear structure for the mean that enables the fitting of a wide range of models to several data types. When the assumption of homoscedasticity is not verified, heteroscedastic models are proposed. Throughout this thesis, we derive an iterative process for fitting the parameters of the models by maximum likelihood. We produce a simple formula to estimate the parameter which defines the transformation of the response variable and the moments of the original dependent variable which generalize previous published results. For the homoscedastic model, we discuss inference, we propose a diagnostic analysis and define a standardized residuals. Finally, to illustrate the theory developed, we use real data sets and we evaluate the results developed through simulations studies.

Keywords: symmetric distribution, transformation parameter, time series. 



\section{Sumário}

Lista de Figuras $\quad$ ix

Lista de Tabelas $\quad$ xi

1 Introdução 1

1.1 Apresentação dos Capítulos . . . . . . . . . . . . . . . . . . . 2

1.2 Alguns Conceitos e Resultados . . . . . . . . . . . . . . . . . . . 2

1.3 Modelos de Regressão Transformados . . . . . . . . . . . . . . . . . . . . . . 4

1.4 Modelos de Regressão Simétricos Transformados . . . . . . . . . . . . . . . 5

1.5 Identificação entre Modelos Não Encaixados . . . . . . . . . . . . . . . . . . . 5

1.5.1 Critérios de Informação . . . . . . . . . . . . . . . . 5

1.5.2 Teste de Modelos Não Encaixados . . . . . . . . . . . . . . . . . 6

2 Modelo de Regressão Simétrico Transformado

Homoscedástico $\quad 9$

2.1 Introdução . . . . . . . . . . . . . . . . . . . . . . . . . . 9

2.2 Modelo Simétrico Homoscedástico . . . . . . . . . . . . . . . . . . . . 9

2.3 Estimação dos Modelos TSARMA . . . . . . . . . . . . . . . . . . . . 10

2.4 Inferência nos Modelos TSARMA . . . . . . . . . . . . . . . . . 14

2.5 Diagnóstico de Modelos TSARMA . . . . . . . . . . . . . . . . . . 15

2.6 Estimativa na Escala Original . . . . . . . . . . . . . . . . . 18

2.7 Simulações . . . . . . . . . . . . . . . . . . . . . . . . . 20

2.8 Aplicação . . . . . . . . . . . . . . . . . . . . . . 30

3 Modelo de Regressão Simétrico Transformado

$\begin{array}{ll}\text { Heteroscedástico } & 37\end{array}$

3.1 Introdução . . . . . . . . . . . . . . . . . . . . . . . . . . . 37

3.2 Modelo Simétrico Heteroscedástico ～. . . . . . . . . . . . . . . . . . . 37

3.3 Modelos TSHARMA Especiais . . . . . . . . . . . . . . . . . . . 42

3.4 Simulações . . . . . . . . . . . . . . . . . . . . . . . . . 44

3.5 Aplicação . . . . . . . . . . . . . . . . . . . . 46

4 Conclusões e Pesquisas Futuras $\quad 51$

Referências Bibliográficas $\quad 55$ 



\section{Lista de Figuras}

2.1 Histogramas e QQ-plot para os estimadores de $\lambda, \beta_{1}, \varphi$ e $\phi$, respectivamente, do modelo normal com transformação. . . . . . . . . . . . . . . . . . . . 22

2.2 Histogramas e QQ-plot para os estimadores de $\beta_{1}, \varphi$ e $\phi$, respectivamente, do modelo normal sem transformação. . . . . . . . . . . . . . . . . . . . . . . . 23

2.3 Histogramas e QQ-plot para os estimadores de $\lambda, \beta_{1}, \varphi$ e $\phi$, respectivamente, do modelo logístico com transformação. . . . . . . . . . . . . . . . . . . . . . 24

2.4 Histogramas e QQ-plot para os estimadores de $\beta_{1}, \varphi$ e $\phi$, respectivamente, do modelo logístico sem transformação. . . . . . . . . . . . . . . . . . . 26

2.5 Histogramas e QQ-plot para os estimadores de $\lambda, \beta_{1}, \varphi$ e $\phi$, respectivamente, do modelo t-Student(5) com transformação. . . . . . . . . . . . . . . . . 26

2.6 Histogramas e QQ-plot para os estimadores de $\beta_{1}, \varphi$ e $\phi$, respectivamente, do modelo t-Student(5) sem transformação. . . . . . . . . . . . . . . . . . . 27

2.7 Gráficos da série, da função de autocorrelação, da função de autocorrelação parcial e box-plot para o conjunto de observações gerados a partir do modelo (2.18). . . 28

2.8 Autocorrelação, autocorrelação parcial dos resíduos e dos resíduos ao quadrado para o modelo AR(1). . . . . . . . . . . . . . . . . . . . . . . . . . . . . 29

2.9 Autocorrelação, autocorrelação parcial dos resíduos e dos resíduos ao quadrado para o modelo TSARMA-t(5). . . . . . . . . . . . . . . . . . . 30

2.10 Série do número anual de linces presos na região do Rio McKenzie, a noroeste do Canadá. . . . . . . . . . . . . . . . . . . . . . 31

2.11 Periodograma, autocorrelação, autocorrelação parcial e boxplot da série do número anual de linces presos na região do Rio McKenzie, a noroeste do Canadá. . . . . . 32

2.12 Autocorrelação, autocorrelação pacial dos resíduos e dos resíduos ao quadrado para o modelo AR(8). . . . . . . . . . . . . . . . . . . . . 32

2.13 Série do número anual de linces presos e estimativas da série na escala original, a partir do modelo AR(8) ajustado. . . . . . . . . . . . . . . . . 33

2.14 Autocorrelação, autocorrelação pacial dos resíduos e dos resíduos ao quadrado para o modelo TSARMA com distribuição normal ajustado. . . . . . . . . . . . . 34

2.15 Autocorrelação, autocorrelação pacial dos resíduos e dos resíduos ao quadrado para o modelo TSARMA com distribuição t-Student(10) ajustado. . . . . . . . . 35

2.16 Série do número anual de linces presos e estimativas da série na escala original, a partir do modelo TSARMA com distribuição t-Student(10) ajustado. . . . . . . . 35

3.1 Histogramas e QQ-plot para os estimadores de $\lambda, \beta_{0}, \varphi$, respectivamente do modelo normal heteroscedástico com transformação. . . . . . . . . . . . . . . . . . . 
3.2 Histogramas e QQ-plot para os estimadores de $\alpha_{0}$ e $\alpha_{1}$, respectivamente do modelo heteroscedástico normal com transformação. . . . . . . . . . . . . . . . 46

3.3 Histogramas e QQ-plot para os estimadores de $\lambda, \beta_{0}, \varphi$, respectivamente do modelo heteroscedástico com transformação e distribuição t-Student(30). . . . . . . . 47

3.4 Histogramas e QQ-plot para os estimadores de $\alpha_{0}$ e $\alpha_{1}$, respectivamente do modelo heteroscedástico com transformação e distribuição t-Student(30). . . . . . . . . . 47

3.5 Série de preços da Petrobras, função de autocorrelação, função de autocorrelação parcial e box-plot. . . . . . . . . . . . . . . . . . . . . 49

3.6 Função de autocorrelação, função de autocorrelação parcial dos resíduos e dos resíduos ao quadrado da modelagem TSARMA com distribuição normal. . . . . . 49

3.7 Função de autocorrelação, função de autocorrelação parcial dos resíduos e dos resíduos ao quadrado da modelagem TSHARMA com distribuição normal. . . . . 50 


\section{Lista de Tabelas}

2.1 Expressões para $W_{f}(u)$ e $W_{f}^{\prime}(u)$ para algumas distribuições simétricas. . . . . . . 12

2.2 Valores para $d_{g}$ e $f_{g}$ para algumas distribuições simétricas. . . . . . . . . . 13

2.3 Valores para os EMV dos respectivos parâmetros $\lambda=1, \beta_{0}=50, \beta_{1}=3, \varphi=$

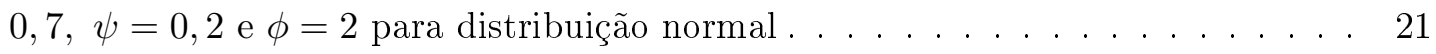

2.4 Valores para os EMV dos respectivos parâmetros $\beta_{1}=3, \varphi=0,7, \psi=0,2$ e $\phi=$ 2 para distribuição normal. . . . . . . . . . . . . . . . . 22

2.5 Valores para os EMV dos respectivos parâmetros $\beta_{1}=3, \varphi=0,7, \psi=0,2$ e $\phi=$ 2 para distribuição logística II. . . . . . . . . . . . . . . . . . . . . 24

2.6 Valores para os EMV dos respectivos parâmetros $\lambda=1, \beta_{0}=50, \beta_{1}=3, \varphi=$

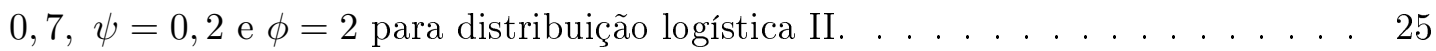

2.7 Valores para os EMV dos respectivos parâmetros $\beta_{1}=3, \varphi=0,7, \psi=0,2$ e $\phi=$

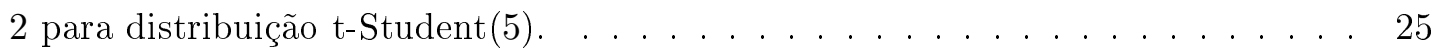

2.8 Valores para os EMV dos respectivos parâmetros $\lambda=1, \beta_{0}=50, \beta_{1}=3, \varphi=$ $0,7, \psi=0,2$ e $\phi=2$ para distribuição t-Student(5). . . . . . . . . 27

2.9 Valores para algumas medidas de comparação entre modelos. . . . . . . . . . . 30

2.10 Valores para algumas medidas de comparação entre modelos. . . . . . . . . . . 34

2.11 Valores para algumas medidas de comparação entre modelos. . . . . . . . . . 36

3.1 Valores para os EMV dos respectivos parâmetros $\lambda=1, \beta_{0}=20, \varphi=0,8, \alpha_{0}=$

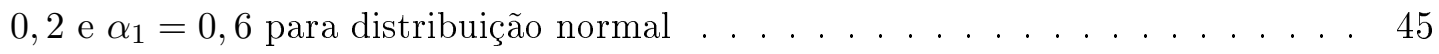

3.2 Valores para os EMV dos respectivos parâmetros $\lambda=1, \beta_{0}=20, \varphi=0,8, \alpha_{0}=$ 0,2 e $\alpha_{1}=0,6$ para distribuição t-Student(30). . . . . . . . . . . . 46

3.3 Valores para as estimativas dos parâmetros para o modelo TSHARMA com distribuição normal. . . . . . . . . . . . . . . . . . . . . . . . . 48 



\section{Capítulo 1}

\section{Introdução}

Inicialmente, Heyde e Feigin (1975) definiram a família exponencial condicional de distribuições. Eles usaram um modelo simples que tinha um único termo autorregressivo, denominado modelo de primeira ordem que, posteriormente, foi mais desenvolvido nos trabalhos de Heyde (1978), Feigin (1981), e por Grunwald, Hyndman, Tedesco e Tweedie (2000).

Zeger e Qaqish (1988) desenvolveram um trabalho que aborda os modelos de Poisson e gama condicionais autorregressivos, adicionando aos modelos de Heyde e Feigin (1975) termos autorregressivos e incluindo o passado e presente de covariáveis. Li (1994) introduziu ao modelo de Zeger e Qaqish (1988) como forma de médias móveis. Com isso, Benjamin et al. (2003) ampliou os trabalhos de Zeger e Qaqish (1988) e Li (1994), considerando o modelo autorregressivo e de médias móveis generalizado (GARMA).

A classe de modelos autorregressivos e de médias móveis generalizados, GARMA, desenvolvida por Benjamin et al. (2003) considera o problema de estender os modelos ARMA de séries temporais para dados cuja distribuição condicional dado o passado do processo pertence à família exponencial. O modelo GARMA pode acomodar o comportamento não estacionário, que pode surgir devido à influência de variáveis exógenas e também, por meio do uso de modelos mistos, permitindo uma parametrização mais parcimoniosa tanto quanto as abordagens de espaço de estados, utilizadas para processos autoregressivos ou processos de médias móveis.

Cordeiro e Andrade (2009) incorporaram a ideia de variável resposta transformada ao modelo GARMA introduzido por Benjamin et al. (2003). Nesse trabalho, assume-se que a distribuição condicional da variável resposta transformada $\left\{Y_{t}^{(\lambda)}, t=1, \ldots, n\right\}$, dado o passado do processo, segue o modelo de dispersão exponencial contínuo. Assim, propõe-se uma classe de modelos ARMA generalizados transformados (TGARMA). Na linha de modelos TGARMA, surgiu como alternativa à modelagem com distribuições condicionais pertencentes à família ex- 
ponencial contínua, o uso de distribuições condicionais simétricas contínuas.

O objetivo geral deste trabalho é desenvolver o modelo TGARMA para distribuições condicionais simétricas contínuas, com e sem heteroscedasticidade, e uma possível função não linear para os parâmetros de regressão.

\section{$1.1 \quad$ Apresentação dos Capítulos}

No Capítulo 2, trataremos do problema de estimação dos parâmetros no modelo autorregressivo e de médias móveis simétrico transformado, TSARMA, o teste da razão de verossimilhanças, bem como a caracterização da distribuição assintótica e, também, proporemos uma análise de diagnóstico, a definição de um resíduo padronizado e uma forma de obter a estimativa da série temporal na escala original. Com o objetivo de observar o comportamento dos estimadores realizaremos algumas simulações. Faremos, também, uma aplicação para avaliar a capacidade preditiva do modelo TSARMA.

No Capítulo 3, abordaremos a estimação dos parâmetros no modelo autorregressivo e de médias móveis simétrico transformado heteroscedástico, TSHARMA, e apresentaremos alguns modelos TSHARMA especiais, uma aplicação e resultados de simulações.

Finalmente, no Capítulo 4, apresentaremos algumas considerações finais e alguns tópicos que poderão ser desenvolvidos em pesquisas futuras.

\subsection{Alguns Conceitos e Resultados}

Nesta seção, apresentamos alguns conceitos e resultados teóricos necessários para o desenvolvimento do texto.

Seja uma variável aleatória $Y$ com suporte em $\mathbb{R}$, com parâmetro de localização $\mu \in \mathbb{R}$ e de dispersão $\phi>0$, com função de densidade de probabilidade expressa por

$$
\pi(y, \mu, \phi)=\frac{1}{\sqrt{\phi}} f\left\{\frac{(y-\mu)^{2}}{\phi}\right\}, \quad y \in \mathbb{R}
$$

para alguma função $f(\cdot)$, denominada função geradora de densidade, sendo $f(u)>0$, para $u>0$ e $\int_{0}^{\infty} u^{\frac{-1}{2}} f(u) d u=1$. Essa condição é necessária para que $\pi(y, \mu, \phi)$ seja uma função de densidade de probabilidade. Denotamos essa variável por $Y \sim S(\mu, \phi)$ e a denominamos de variável aleatória simétrica.

A família de distribuições simétricas permite uma extensão da distribuição normal para modelagem estatística de dados reais envolvendo distribuições com caudas mais ou menos pesadas 
do que a cauda da distribuição normal. Essa família engloba a distribuição normal, Cauchy, tStudent, t-Student generalizada, logística I, logística II, logística generalizada, exponencial dupla, exponencial potência, potência estendida, Kotz, Kotz generalizada, normal contaminada, entre outras. A seguir, estão listados alguns casos especiais dessa família.

(i) Normal: $f(u)=(2 \pi)^{-\frac{1}{2}} \exp \left(-\frac{u}{2}\right)$;

(ii) Cauchy: $f(u)=\{\pi(1+u)\}^{-1}$;

(iii) t-Student: $f(u)=\nu^{\frac{\nu}{2}} B\left(\frac{1}{2}, \frac{\nu}{2}\right)^{-1}(\nu+u)^{-\frac{(\nu+1)}{2}}, \nu>0$, em que $B(\cdot, \cdot)$ é a função beta;

(iv) t-Student Generalizada: $f(u)=s^{\frac{r}{2}} B\left(\frac{1}{2}, \frac{r}{2}\right)^{-1}(s+u)^{-\frac{(r+1)}{2}}, s, r>0$, sendo que a distribuição t-Student é um caso particular quando $s=r=\nu$ e a distribuição Cauchy outro caso particular quando $s=r=1$;

(v) Logística I: $f(u)=c \exp (-u)[1+\exp (-u)]^{-2}$, em que $c \approx 1.484300029$ é a constante normalizadora a qual segue de $\int_{0}^{\infty} u^{-\frac{1}{2}} f(u) d u=1$;

(vi) Logística II: $f(u)=\exp \left(-u^{\frac{1}{2}}\right)\left[1+\exp \left(-u^{-\frac{1}{2}}\right)\right]^{-2}$;

(vii) Logística Generalizada: $f(u)=c B(m, m)^{-1}\left\{\exp (-c \sqrt{u})[1+\exp (-c \sqrt{u})]^{-2}\right\}^{m}, m>0, c>$ 0 . A densidade logística II corresponde ao caso $m=1, c=1$;

(viii) Kotz: $f(u)=\frac{r^{\frac{2 m-1}{2}}}{\Gamma\left(\frac{2 m-1}{2}\right)} u^{m-1} \exp (-r u), r>0, m \geq 1$, (Kotz, 1975), em que $\Gamma(\cdot)$ é a função gama. Se $m=1$, esta distribuição se reduz a distribuição normal com média $\mu$ e variância $\frac{\phi^{2}}{2 r}$

(ix) Kotz Generalizada: $f(u)=\frac{s r^{\frac{2 m-1}{2 s}}}{\Gamma\left(\frac{2 m-1}{2 s}\right)} u^{m-1} \exp \left(-r u^{s}\right), r, s>0, m \geq 1$. Para $s=1$, reduz-se a distribuição de Kotz e para $m=s=1$ e $r=\frac{1}{2}$, retornamos a distribuição $\mathrm{N}\left(\mu, \phi^{2}\right)$. Quando $m=1, r=\frac{1}{2}$ e $s=\frac{1}{1+k}$, coincide com a distribuição exponencial potência, que será definida no caso (xii);

(x) Normal Contaminada: $f(u)=(1-\varepsilon) \frac{1}{\sqrt{2 \pi}} \exp \left(-\frac{u}{2}\right)+\varepsilon \frac{1}{\sqrt{2 \pi} \sigma} \exp \left(-\frac{u}{2 \sigma^{2}}\right), \sigma>0,0 \leq \varepsilon \leq 1$;

(xi) Exponencial Dupla: $f(u)=\frac{\exp (-\sqrt{u})}{2}$;

(xii) Exponencial Potência: $f(u)=c(r) \exp \left(-\frac{1}{2} u^{\frac{1}{1+r}}\right),-1<r \leq 1$, em que $c(r)=\Gamma(1+$ $\left.\frac{(1+r)}{2}\right) 2^{\frac{1+(1+r)}{2}}$ (Box e Tiao, 1973); 
(xiii) Potência Estendida: $f(u)=K(c, \lambda) \exp \left[-\frac{1}{2} c \rho_{\lambda}\left(1+\frac{u}{c-1}\right)\right]$, em que $K(c, \lambda)$ é a constante normalizadora, $c>1, \lambda \geq 0$ e $\rho_{\lambda}(v)= \begin{cases}\frac{v^{\lambda}-1}{\lambda}, & \text { se } \lambda>0, \\ \log v, & \text { se } \lambda=0 .\end{cases}$

(Albert et al., 1991).

Se $Y \sim S(\mu, \phi)$, então, a função característica de $Y, \varsigma(t)=\mathrm{E}[\exp (i t Y)]$ é expressa por $\exp (i t \mu) \times \varphi\left(t^{2} \phi\right), t \in \mathbb{R}$, para alguma função $\varphi, \operatorname{com} \varphi(u) \in \mathbb{R}$ para $u>0$. Quando existem, $\mathrm{E}(Y)=\mu$ e $\operatorname{VAR}(Y)=\kappa \phi$, em que $\kappa>0$ é uma constante igual a $\kappa=-2 \varphi^{\prime}(0)$, com $\varphi^{\prime}(0)=$ $\left.\frac{d \varphi(u)}{d u}\right|_{u=0}$ e que não depende dos parâmetros $\mu$ e $\phi$. Se $u^{-\frac{1}{2}(k+1)} f(u)$ for integrável, então, o $k$-ésimo momento de $Y$ existe, ver Kelker (1970).

\subsection{Modelos de Regressão Transformados}

Suponha um par de séries temporais distribuídas conjuntamente $\left\{\left(x_{t}, Y_{t}\right), t=1, \ldots, T\right\}$, em que $Y_{t}$ é a série temporal resposta de interesse e $x_{t}$ é uma covariável. Considere uma família paramétrica de transformações para a variável resposta $Y_{t}$ como

$$
Y_{t}^{(\lambda)}=\Lambda\left(Y_{t} ; \lambda\right)
$$

em que $\Lambda\left(Y_{t} ; \lambda\right)$ é uma função real de $Y_{t}$, conhecida e estritamente monótona que depende de um parâmetro escalar $\lambda$, desconhecido, o qual define uma transformação particular.

Uma das transformações mais conhecidas, proposta por Box e Cox (1964), que é adequada somente para dados positivos contínuos e transforma o valor observado $Y \mathrm{em}$

$$
Y^{(\lambda)}=\left\{\begin{array}{ccc}
\frac{Y^{\lambda}-1}{\lambda}, & \text { se } & \lambda \neq 0 \\
\log Y, & \text { se } & \lambda=0
\end{array}\right.
$$

supõe que existe um valor de $\lambda$ tal que $Y^{(\lambda)}$ seja, aproximadamente, normal com variância constante e satisfazendo um modelo de regressão linear $\mu=X \beta$. Contudo, na prática, isso ocorrerá raramente. Manly (1976) propôs a transformação exponencial para transformar observações negativas $Y$ em

$$
Y^{(\lambda)}=\left\{\begin{array}{ccc}
\frac{\left(e^{\lambda Y}-1\right)}{\lambda}, & \text { se } \lambda \neq 0 \\
\log Y, & \text { se } \lambda=0
\end{array}\right.
$$

Outras formas de transformações potência podem ser encontradas em Sakia (1992). 


\subsection{Modelos de Regressão Simétricos Transformados}

O conjunto $H_{t}=\left\{x_{t}, \ldots, x_{1}, y_{t-1}^{(\lambda)}, \ldots, y_{1}^{(\lambda)}, \mu_{t-1}, \ldots, \mu_{1}\right\}$ representa os valores passados da série transformada, das suas médias e os possíveis valores presentes e passados das covariáveis.

Suponha que a distribuição condicional da variável resposta transformada $\left\{Y_{t}^{(\lambda)}, t=1, \ldots, T\right\}$, dado o passado do processo $H_{t}$, segue uma distribuição simétrica contínua com $\mathrm{E}\left(Y_{t}^{(\lambda)} \mid H_{t}\right)=$ $\mu_{t} \in \mathbb{R}$. Quando $\operatorname{VAR}\left(Y_{t}^{(\lambda)} \mid H_{t}\right)=\kappa \phi>0$, teremos um modelo simétrico homoscedástico e quando $\operatorname{VAR}\left(Y_{t}^{(\lambda)} \mid H_{t}\right)=\kappa \phi_{t}>0$, teremos um modelo simétrico heteroscedástico. Esses dois modelos serão tratados nos capítulos que seguem.

\subsection{Identificação entre Modelos Não Encaixados}

Dois modelos condicionais $F_{\theta}=\{f(y \mid \mathbf{x}, \theta) ; \theta \in \mathbf{\Theta}\}$ e $G_{\gamma}=\{g(y \mid \mathbf{x}, \gamma) ; \gamma \in \boldsymbol{\Gamma}\}$ são ditos não encaixados, se e somente se,

$$
F_{\theta} \cap G_{\gamma}=\varnothing,
$$

ou seja, se nenhum deles pode ser representado como um caso especial do outro. Definições formais são apresentadas em Pesaram (1987) e Vuong (1989).

\subsubsection{Critérios de Informação}

Para comparação entre modelos não encaixados baseados em máxima verossimilhança, muitos autores como, por exemplo, Akaike (1973) propuseram critérios de seleção de modelos baseados no logaritmo da função de verossimilhança estimado. Esse critério penaliza modelos com grande número de parâmetros, $k$, isto porque esperamos que o logaritmo da função de verossimilhança cresça com o número de parâmetros adicionados no modelo. Essa penalidade também pode ser uma função de $n$, o número de observações.

Akaike (1973) propôs o critério de informação

$$
\mathrm{AIC}=-2 \hat{l}(\cdot)+2 k
$$

em que $\hat{l}$ é o logaritmo da função de verossimilhança maximizado sob o modelo considerado.

Um valor baixo para AIC é considerado como representativo de um melhor ajuste e os modelos são selecionados visando a obter um mínimo AIC. O termo critério de informação é usado, pois o logaritmo da função de verossimilhança é relativamente próximo ao critério de informação de Kullback-Leibler (1951). 
Existem várias correções para melhorar o comportamento do AIC, no sentido de diminuir a probabilidade de selecionar uma ordem maior do que a necessária. Modificações do AIC incluem o critério de informação Bayesiano

$$
\mathrm{BIC}=-2 \hat{l}(\cdot)+(\ln n) k
$$

proposto por Schwarz (1978) e o critério de informação de Akaike consistente

$$
\mathrm{CAIC}=-2 \hat{l}(\cdot)+(1+\ln n) k .
$$

Esses três critérios dão, cada vez mais, maiores penalidades em $k$ e $n$. Por exemplo, suponha que desejamos comparar dois modelos, sendo que um modelo tem um parâmetro a mais do que o outro, isto é $\Delta k=1$, e o tamanho da amostra é $n=1000$, assim $\ln n=6,9$. Para o modelo maior ser preferido ele necessita aumentar $2 \hat{l}(\cdot)$ em 1,0 se usarmos o AIC, 6, 9 se usarmos o BIC, e 7,9 se usarmos o CAIC.

\subsubsection{Teste de Modelos Não Encaixados}

Existe uma literatura substâncial sobre discriminação entre modelos não encaixados usando como base testes de hipóteses, embora testes não usuais. Sejam dois modelos não encaixados, $F_{\theta}$ com densidade $f\left(y_{i} \mid \mathbf{x}_{\mathbf{i}}, \theta\right)$ e o modelo $G_{\gamma}$ com densidade $g\left(y_{i} \mid \mathbf{x}_{\mathbf{i}}, \gamma\right)$.

A estatística, LR, da razão de verossimilhanças para o modelo $F_{\theta}$ contra o modelo $G_{\gamma}$ é

$$
\operatorname{LR}(\hat{\theta}, \hat{\gamma}) \equiv l_{f}(\hat{\theta})-l_{g}(\hat{\gamma})=\sum_{i=1}^{n} \ln \frac{f\left(y_{i} \mid \mathbf{x}_{\mathbf{i}}, \hat{\theta}\right)}{g\left(y_{i} \mid \mathbf{x}_{\mathbf{i}}, \hat{\gamma}\right)}
$$

No caso especial em que os modelos são encaixados, $F_{\theta} \subset G_{\gamma}$, nós obtemos o resultado usual que

$2 \times \operatorname{LR}(\hat{\theta}, \hat{\gamma})$ tem distribuição qui-quadrado sob a hipótese nula, $F_{\theta}=G_{\gamma}$. Para o caso de modelos não encaixados, ou seja, $F_{\theta} \nsubseteq G_{\gamma}$ e $F_{\theta} \nsupseteq G_{\gamma}$ a distribuição qui-quadrado não é apropriada.

Cox $(1961,1962 a)$ propôs resolver este problema aplicando o teorema central do limite sob a suposição que $F_{\theta}$ é o modelo verdadeiro. Esta abordagem é difícil para implementar, pois requer uma obtenção analítica de

$$
\mathrm{E}_{f}\left[\ln \frac{f\left(y_{i} \mid \mathbf{x}_{\mathbf{i}}, \theta\right)}{g\left(y_{i} \mid \mathbf{x}_{\mathbf{i}}, \gamma\right)}\right]
$$

em que $\mathrm{E}_{f}$ denota a esperança com relação à densidade $f\left(y_{i} \mid \mathbf{x}_{\mathbf{i}}, \theta\right)$. Além disso, se uma estatística 
de teste similar é obtida sob a suposição que $G_{\gamma}$ é o modelo verdadeiro, podemos rejeitar o modelo $F_{\theta}$ em favor do $G_{\gamma}$ e rejeitar o modelo $G_{\gamma}$ em favor do $F_{\theta}$.

Vuong (1989) apresentou uma solução interessante para resolver esse problema. Vuong (1989) segue Akaike $(1973,1974)$ e considera o critério de informação de Kullback-Leibler (1951), KLIC, o qual mede a distância entre uma distribuição qualquer e a distribuição verdadeira. Dessa forma, Vuong (1989) faz distinção entre modelos baseando-se nas distâncias entre cada modelo e o verdadeiro processo gerador dos dados, o qual tem densidade $h_{0}\left(y_{i} \mid X_{i}\right)$, em que a distância é medida usando o critério de informação de Kullback-Liebler.

Ele propôs a estatística

$$
\begin{aligned}
\mathrm{T}_{\mathrm{LR}, \mathrm{NN}}= & \frac{1}{\sqrt{n}} \sum_{i=1}^{n} \ln \frac{f\left(y_{i} \mid \mathbf{x}_{\mathbf{i}}, \hat{\theta}\right)}{g\left(y_{i} \mid \mathbf{x}_{\mathbf{i}}, \hat{\gamma}\right)} \div \\
& \left\{\frac{1}{n} \sum_{i=1}^{n}\left(\ln \frac{f\left(y_{i} \mid \mathbf{x}_{\mathbf{i}}, \hat{\theta}\right)}{g\left(y_{i} \mid \mathbf{x}_{\mathbf{i}}, \hat{\gamma}\right)}\right)^{2}-\left(\frac{1}{n} \sum_{i=1}^{n} \ln \frac{f\left(y_{i} \mid \mathbf{x}_{\mathbf{i}}, \hat{\theta}\right)}{g\left(y_{i} \mid \mathbf{x}_{\mathbf{i}}, \hat{\gamma}\right)}\right)^{2}\right\} \\
= & \frac{1}{\sqrt{n}} \frac{\operatorname{LR}(\hat{\theta}, \hat{\gamma})}{\hat{\omega}^{2}},
\end{aligned}
$$

em que

$$
\hat{\omega}^{2}=\frac{1}{n} \sum_{i=1}^{n}\left(\ln \frac{f\left(y_{i} \mid \mathbf{x}_{\mathbf{i}}, \hat{\theta}\right)}{g\left(y_{i} \mid \mathbf{x}_{\mathbf{i}}, \hat{\gamma}\right)}\right)^{2}-\left(\frac{1}{n} \sum_{i=1}^{n} \ln \frac{f\left(y_{i} \mid \mathbf{x}_{\mathbf{i}}, \hat{\theta}\right)}{g\left(y_{i} \mid \mathbf{x}_{\mathbf{i}}, \hat{\gamma}\right)}\right)^{2}
$$

é uma estimativa da variância de $\frac{1}{\sqrt{n}} \operatorname{LR}(\hat{\theta}, \hat{\gamma})$. Uma alternativa assintoticamente equivalente para (1.6) e (1.7) usa $\widetilde{\omega}^{2}=\frac{1}{n} \sum_{i=1}^{n}\left\{\ln \frac{f\left(y_{i} \mid \mathbf{x}_{\mathbf{i}}, \hat{\theta}\right)}{g\left(y_{i} \mid \mathbf{x}_{\mathbf{i}}, \hat{\gamma}\right)}\right\}^{2}$.

Para modelos não encaixados

$$
\mathrm{T}_{\mathrm{LR}, \mathrm{NN}} \stackrel{d}{\longrightarrow} N(0,1)
$$

sob

$$
\mathrm{H}_{0}: \mathrm{E}_{h}\left[\ln \frac{f\left(y_{i} \mid \mathbf{x}_{\mathbf{i}}, \theta\right)}{g\left(y_{i} \mid \mathbf{x}_{\mathbf{i}}, \gamma\right)}\right]=0
$$

em que $\mathrm{E}_{h}$ denota o valor esperado com relação ao (desconhecido) processo gerador dos dados $h_{0}\left(y_{i} \mid \mathbf{x}_{\mathbf{i}}\right)$. Portanto, rejeita-se ao nível de 0,05 a hipótese nula de equivalência dos modelos em favor ao modelo $F_{\theta}$ sendo melhor (ou pior) do que o modelo $G_{\gamma}$ se $\mathrm{T}_{\mathrm{LR}, \mathrm{NN}}>z_{, 05}$ (ou se $\left.\mathrm{T}_{\mathrm{LR}, \mathrm{NN}}<-z_{, 05}\right)$. A hipótese nula não é rejeitada se $\left|\mathrm{T}_{\mathrm{LR}, \mathrm{NN}}\right| \leqslant z, 025$. 



\section{Capítulo 2}

\section{Modelo de Regressão Simétrico Transformado Homoscedástico}

\subsection{Introdução}

A suposição de normalidade da variável resposta para um modelo é questionável quando os dados contêm outliers e estimativas dos parâmetros podem ser enganosas. Em vista disto, consideramos uma família geral de transformações monotônicas e combinamos a ideia de Box-Cox a uma família mais geral de distribuições simétricas que mantem a suposição de homoscedasticidade com uma possível função não linear dos parâmetros.

Neste capítulo, vamos desenvolver o modelo TGARMA para distribuições condicionais simétricas contínuas sem heteroscedasticidade e uma possível função não linear para os parâmetros de regressão.

\subsection{Modelo Simétrico Homoscedástico}

Suponha que a distribuição condicional da variável resposta transformada $\left\{Y_{t}^{(\lambda)}, t=1, \ldots, T\right\}$ dado o passado do processo $H_{t}$ segue uma distribuição simétrica contínua, $Y_{t}^{(\lambda)} \mid H_{t} \sim S\left(\mu_{t}, \phi\right)$, ou seja, $\mathrm{E}\left(Y_{t}^{(\lambda)} \mid H_{t}\right)=\mu_{t} \in \mathbb{R}$ e $\operatorname{VAR}\left(Y_{t}^{(\lambda)} \mid H_{t}\right)=\kappa \phi>0$, Assim,

$$
\pi\left(y_{t}^{(\lambda)} \mid H_{t}, \mu_{t}, \phi\right)=\frac{1}{\sqrt{\phi}} f\left\{\phi^{-1}\left(y_{t}^{(\lambda)}-\mu_{t}\right)^{2}\right\}
$$

Considere o modelo

$$
y_{t}^{(\lambda)}=\mu_{t}+\sqrt{\phi} \varepsilon_{t}, t=1, \ldots, T, \quad \varepsilon_{t} \sim S(0,1)
$$

satisfazendo (2.1) e 


$$
\mu_{t}=g^{-1}\left(\eta_{t}\right)
$$

em que

$$
\eta_{t}=x_{t} \beta+\sum_{i=1}^{p} \varphi_{i}\left\{g\left(y_{t-i}^{(\lambda)}\right)-x_{t-i} \beta\right\}+\sum_{i=1}^{q} \psi_{i}\left\{g\left(y_{t-i}^{(\lambda)}\right)-\eta_{t-i}\right\}, \quad t=1, \ldots, T .
$$

O modelo Autorregressivo e de Médias Móveis Simétrico Transformado, TSARMA, é definido pela família de transformações (1.1), pela distribuição simétrica (2.1), pelo componente sistemático (2.2) e pela estrutura de regressão para a média (2.3).

\subsection{Estimação dos Modelos TSARMA}

Os parâmetros do modelo são $\beta_{m \times 1}, \varphi_{p \times 1}, \psi_{q \times 1}$ e $\phi$. Os estimadores desses parâmetros serão obtidos pelo método de máxima verossimilhança condicional às primeiras $k$ observações transformadas, em que $k=\max \{p, q\}$. A função de verossimilhança é

$$
L(\nu, \phi, \lambda)=\prod_{t=k+1}^{T} \pi\left(y_{t}^{(\lambda)} \mid H_{t}\right) \times J\left(\lambda, y_{t}\right)
$$

sendo $J\left(\lambda, y_{t}\right)=\left|\frac{d \Lambda\left(y_{t} ; \lambda\right)}{d y_{t}}\right|$ o Jacobiano da transformação de $y_{t}$ em $y_{t}^{(\lambda)}$ e $\nu=\left(\beta^{T}, \varphi^{T}, \psi^{T}\right)^{T}$. Assim, o logaritmo da função de verossimilhança condicional é expresso por

$$
l(\nu, \phi, \lambda)=-\frac{(T-k)}{2} \log (\phi)+\sum_{t=k+1}^{T} \log \left[f\left\{\phi^{-1}\left(y_{t}^{(\lambda)}-\mu_{t}\right)^{2}\right\}\right]+\sum_{t=k+1}^{T} \log \left\{J\left(\lambda, y_{t}\right)\right\} .
$$

Usando a transformação de Box-Cox na variável resposta, tem-se $\log \left\{J\left(\lambda, y_{t}\right)\right\}=(\lambda-$ 1) $\log \left|y_{t}\right|$.

A função $l(\nu, \phi, \lambda)$ é suposta ser regular (Cox e Hinkley, 1974) com relação a $\nu$ e $\phi$. Condições regulares são encontradas, também, em Serfling (1980, p.144). Para obter a função escore e a matriz de informação de Fisher precisamos derivar $l(\nu, \phi, \lambda)$ com relação aos parâmetros desconhecidos. Supomos aqui que tais derivadas existem. Contudo, algumas distribuições simétricas não satisfazem as condições usuais de regularidade, por exemplo, Kotz, Kotz generalizada e exponencial dupla.

Podemos reescrever o modelo geral para a média $(2.3)$, para $t=k+1, \ldots, T$, como 


$$
\eta=\left(\begin{array}{lll}
X & A & B
\end{array}\right)\left(\begin{array}{l}
\beta \\
\varphi \\
\psi
\end{array}\right)
$$

em que

$$
\begin{aligned}
\eta & =\left(\begin{array}{c}
\eta_{k+1} \\
\eta_{k+2} \\
\vdots \\
\eta_{T}
\end{array}\right)_{(T-k) \times 1}, X=\left(\begin{array}{c}
x_{k+1} \\
x_{k+2} \\
\vdots \\
x_{T}
\end{array}\right)_{(T-k) \times m}, \\
A & =\left(\begin{array}{ccc}
g\left(y_{k}^{(\lambda)}\right)-x_{k} \beta & \cdots & g\left(y_{k+1-p}^{(\lambda)}\right)-x_{k+1-p} \beta \\
g\left(y_{k+1}^{(\lambda)}\right)-x_{k+1} \beta & \cdots & g\left(y_{k+2-p}^{(\lambda)}\right)-x_{k+2-p} \beta \\
\vdots & \ddots & \vdots \\
g\left(y_{T-1}^{(\lambda)}\right)-x_{T-1} \beta & \cdots & g\left(y_{T-p}^{(\lambda)}\right)-x_{T-p} \beta
\end{array}\right)_{(T-k) \times p} \\
& =\left(\begin{array}{ccc}
g\left(y_{k}^{(\lambda)}\right)-\eta_{k} & \cdots & g\left(y_{k+1-q}^{(\lambda)}\right)-\eta_{k+1-q} \\
g\left(y_{k+1}^{(\lambda)}\right)-\eta_{k+1} & \cdots & g\left(y_{k+2-q}^{(\lambda)}\right)-\eta_{k+2-q} \\
\vdots & \ddots & \vdots \\
g\left(y_{T-1}^{(\lambda)}\right)-\eta_{T-1} & \cdots & g\left(y_{T-q}^{(\lambda)}\right)-\eta_{T-q}
\end{array}\right)_{(T-k) \times q}
\end{aligned}
$$

Assim, a estrutura de regressão para a média pode ser reescrita como

$$
\eta=M^{(\lambda)} \nu
$$

em que

$$
M^{(\lambda)}=\left(\begin{array}{ccc}
X & A & B
\end{array}\right), \nu=\left(\begin{array}{l}
\beta \\
\varphi \\
\psi
\end{array}\right)
$$

Para maximizar o logaritmo da função de verossimilhança condicional (2.4), assumiremos, inicialmente, que o parâmetro de transformação, $\lambda$, é fixo. Assim, dado o parâmetro de transformação $\lambda$, as estimativas de máxima verossimilhança condicional são $\hat{\nu}^{(\lambda)}=\left(\begin{array}{c}\hat{\beta}^{(\lambda)} \\ \hat{\varphi}^{(\lambda)} \\ \hat{\psi}^{(\lambda)}\end{array}\right), \hat{\eta}^{(\lambda)}=$ 


\begin{tabular}{ccc}
\hline Distribuição & $W_{f}(u)$ & $W_{f}^{\prime}(u)$ \\
\hline Normal & $-\frac{1}{2}$ & 0 \\
t-Student & $-\frac{\nu+1}{2(\nu+u)}$ & $\frac{\nu+1}{2(\nu+u)^{2}}$ \\
t-Student generalizada & $-\frac{r+1}{2(s+u)}$ & $\frac{r+1}{2(s+u)^{2}}$ \\
Logística I & $-\tanh \left(\frac{u}{2}\right)$ & $-\operatorname{sech}\left(\frac{u}{2}\right) / 2$ \\
Logística II & $-\frac{\exp (-\sqrt{u})-1}{(-2 \sqrt{u})[1+\exp (-\sqrt{u})]}$ & $\frac{2 \exp (-\sqrt{u}) \sqrt{u}+\exp (-2 \sqrt{u})-1}{-4 u^{3 / 2}[1+\exp (-\sqrt{u})]^{2}}$ \\
Logística generalizada & $-\frac{\alpha m[\exp (-\alpha \sqrt{u})-1]}{(-2 \sqrt{u})[1+\exp (-\alpha \sqrt{u})]}$ & $-\frac{\alpha m}{4} \frac{2 \alpha \exp (-\alpha \sqrt{u}) \sqrt{u}+\exp (-2 \alpha \sqrt{u})-1}{u^{3 / 2}[1+\exp (-\alpha \sqrt{u})]^{2}}$ \\
Exponencial potência & $-\frac{1}{2(1+k) u^{k /(k+1)}}$ & $\frac{k}{(1+k)^{2} 2 u(2 k+1) /(1+k)}$ \\
& &
\end{tabular}

Tabela 2.1: Expressões para $W_{f}(u)$ e $W_{f}^{\prime}(u)$ para algumas distribuições simétricas.

$\hat{M}^{(\lambda)} \hat{\nu}^{(\lambda)}, \hat{\mu}^{(\lambda)}=g^{-1}\left(\hat{\eta}^{(\lambda)}\right)$ e $\hat{\phi}^{(\lambda)}$ de $\nu=\left(\begin{array}{l}\beta \\ \varphi \\ \psi\end{array}\right), \eta, \mu$, e $\phi$, respectivamente.

As funções escore para $\nu$ e $\phi$ têm, respectivamente, as formas

$U_{\nu}(\nu, \phi)=\phi^{-1} M^{(\lambda) T} H D^{(\lambda)}\left(y^{(\lambda)}-\mu\right) \quad$ e $\quad U_{\phi}(\nu, \phi)=\frac{1}{2 \phi}\left[-(T-k)+\phi^{-1}\left(y^{(\lambda)}-\mu\right)^{T} D^{(\lambda)}\left(y^{(\lambda)}-\mu\right)\right]$,

em que $\quad H=\operatorname{diag}\left\{\mu_{k+1}^{\prime}, \ldots, \mu_{T}^{\prime}\right\}, \quad \mu_{i}^{\prime}=\frac{d \mu_{i}}{d \eta_{i}}, \quad D^{(\lambda)}=\operatorname{diag}\left\{v_{k+1}, \ldots, v_{T}\right\}, \quad v_{i}=-2 W_{f}\left(u_{i}\right)=$

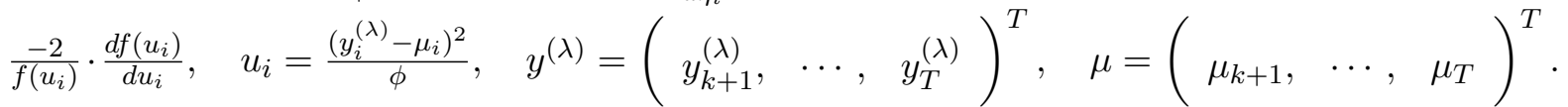

Expressões para $W_{f}(u)$ e $W_{f}^{\prime}(u)$ para algumas distribuições simétricas são apresentadas na Tabela 2.1.

A matriz de informação esperada de Fisher para $(\nu, \phi)$ é bloco-diagonal,

$$
K=\left(\begin{array}{cc}
K_{\nu \nu} & 0 \\
0 & K_{\phi \phi}
\end{array}\right)
$$

ou seja, os vetores de parâmetros $\nu$ e $\phi$ são globalmente ortogonais. Lange, Little e Taylor (1989) apresentam inferência básica para modelos $t$-multivariados com números de graus de liberdade desconhecidos. Nesse artigo, pode-se encontrar uma maneira de calcular a matriz de informação esperada de Fisher para $(\nu, \phi)$. As matrizes de informação esperada de Fisher $K_{\nu \nu}$ e $K_{\phi \phi}$ para 


\begin{tabular}{ccc}
\hline Distribuição & $d_{g}$ & $f_{g}$ \\
\hline Normal & $\frac{1}{4}$ & $\frac{3}{4}$ \\
t-Student & $\frac{\nu+1}{4(\nu+3)}$ & $\frac{3(\nu+1)}{4(\nu+3)}$ \\
t-Student generalizada & $\frac{r(r+1)}{4 s(r+3)}$ & $\frac{3(r+1)}{4(r+3)}$ \\
Logística I & 0,369310044 & 1,003445984 \\
Logística II & $\frac{1}{12}$ & 0,60749 \\
Logística generalizada & $\frac{\alpha^{2} m^{2}}{4(2 m+1)}$ & $\frac{2 m\left(2+m^{2} \psi^{\prime}(m)\right)}{4(2 m+1)}$ \\
Exponencial potência & $\frac{\Gamma\{(3-k) / 2\}}{4\left(2^{k-1}\right)(1+k)^{2} \Gamma\{(k+1) / 2\}}$ & $\frac{k+3}{4(k+1)}$ \\
\hline
\end{tabular}

Tabela 2.2: Valores para $d_{g}$ e $f_{g}$ para algumas distribuições simétricas.

$\nu$ e $\phi$ são expressas, respectivamente, por

$$
K_{\nu \nu}=4 d_{g} \phi^{-1} M^{(\lambda) T} H^{2} M^{(\lambda)} \quad \text { e } \quad K_{\phi \phi}=\frac{T-k}{4 \phi^{2}}\left(4 f_{g}-1\right),
$$

em que $\quad d_{g}=\mathrm{E}\left\{W_{f}^{2}\left(U^{2}\right) U^{2}\right\} \quad$ e $f_{g}=\mathrm{E}\left\{W_{f}^{2}\left(U^{2}\right) U^{4}\right\}, \quad$ sendo $\quad U \sim S(0,1)$. Alguns valores de $d_{g}$ e $f_{g}$ são descritos na Tabela 2.2 e mais detalhes podem ser encontrados em Cysneiros e Paula (2005).

Um processo iterativo para calcular as estimativas de máxima verossimilhança, EMVs, de $\nu$ e $\phi$ é o método escore de Fisher. As EMVs $\hat{\nu}^{(\lambda)}$ e $\hat{\phi}^{(\lambda)}$ podem ser obtidas resolvendo-se o seguinte sistema de equações:

$$
\begin{aligned}
& \nu^{(\lambda)(m+1)}=\left[M^{(\lambda) T(m)} H^{(\lambda)(m) 2} M^{(\lambda)(m)}\right]^{-1} M^{(\lambda) T(m)} H^{(\lambda)(m) 2} z_{\nu}^{(\lambda)(m)}, \\
& \phi^{(\lambda)(m+1)}=\frac{1}{T-k}\left[y^{(\lambda)}-\mu\left(\nu^{(m+1)}\right)\right]^{T} D^{(\lambda)(m+1)}\left[y^{(\lambda)}-\mu\left(\nu^{(m+1)}\right)\right],
\end{aligned}
$$

em que $z_{\nu}^{(\lambda)(m)}$ é um vetor $(T-k) \times 1$ expresso por

$$
z_{\nu}^{(\lambda)(m)}=\eta^{(m)}+\left(4 d_{g}\right)^{-1} H^{(\lambda)(m)(-1)} D^{(\lambda)(m)}\left(y^{(\lambda)}-\mu^{(\lambda)(m)}\right) .
$$

Substituindo as estimativas de máxima verossimilhança condicional $\hat{\nu}^{(\lambda)}$ e $\hat{\phi}^{(\lambda)}$ na equação (2.4), obtém-se o logaritmo da função de verossimilhança perfilada para $\lambda$, 


$$
l(\lambda)=-\frac{(T-k)}{2} \log \left(\hat{\phi}^{(\lambda)}\right)+\sum_{t=k+1}^{T} \log \left[f\left\{\hat{\phi}^{(\lambda)(-1)}\left(y_{t}^{(\lambda)}-\hat{\mu}_{t}^{(\lambda)}\right)^{2}\right\}\right]+\sum_{t=k+1}^{T} \log \left\{J\left(\lambda, y_{t}\right)\right\} .
$$

Seja $s_{t}^{(\lambda) 2}=f\left\{\left(\hat{\phi}_{t}^{(\lambda)(-1)}\left(y_{t}^{(\lambda)}-\hat{\mu}_{t}^{(\lambda)}\right)^{2}\right)\right\}$ e defina as médias geométricas

$$
\tilde{s}^{(\lambda) 2}=\left(\prod_{t=k+1}^{T} s_{t}^{(\lambda) 2}\right)^{\frac{1}{T-k}} \text { e } \tilde{J}^{(\lambda)}=\left(\prod_{t=k+1}^{T} J\left(\lambda, y_{t}\right)\right)^{\frac{1}{T-k}} .
$$

Então, o logaritmo da função de verossimilhança perfilada fica reduzido a

$$
l_{p}(\lambda)=(T-k) \log \left(\frac{\tilde{s}^{(\lambda) 2} \tilde{J}^{(\lambda)}}{\sqrt{\hat{\phi}^{(\lambda)}}}\right) .
$$

O logaritmo da função de verossimilhança perfilada (2.6) é maximizada numericamente por meio do gráfico de $l_{p}(\lambda)$ versus $\lambda$, usando uma grade de valores para $\lambda$. Para maximizar (2.6), necessitamos encontrar um valor de $\lambda$ que minimize a razão

$$
\hat{\lambda}=\arg \min _{\lambda}\left(\frac{\sqrt{\hat{\phi}^{(\lambda)}}}{\tilde{s}^{(\lambda) 2} \tilde{J}^{(\lambda)}}\right)
$$

Podemos substituir as EMVs nas equações (2.5) e encontrar as estimativas irrestritas de $\hat{\nu}=\hat{\nu}^{(\hat{\lambda})}$ e $\hat{\phi}=\hat{\phi}^{(\hat{\lambda})}$ e, assim, o logaritmo da função de verossimilhança maximizado $\hat{l}=l_{p}(\hat{\lambda})$ segue de (2.6) para o modelo considerado.

\subsection{Inferência nos Modelos TSARMA}

No modelo clássico de regressão em que a variável resposta tem distribuição normal e a função de ligação é a identidade, as distribuções dos estimadores dos parâmetros e das estatísticas usadas para verificar o ajuste do modelo aos dados podem ser determinadas exatamente. Geralmente, contudo, a obtenção de distribuições exatas nos modelos lineares generalizados (MLGs) é muito complicada e resultados assintóticos são usados. Esses resultados, porém, dependem de algumas condições de regularidade e do número de observações independentes mas, em particular, para os MLGs essas condições são satisfeitas (Fahrmeir e Kaufmann, 1985).

O procedimento descrito para estimar o vetor de parâmetros $(\nu, \phi)$, no modelo TSARMA, executa a estimação de máxima verossimilhança usando, iterativamente, mínimos quadrados reponderados condicional ao parâmetro de transformação, $\lambda$, (Green, 1984). No modelo TSARMA, 
como esperado, a obtenção de distribuições exatas dos estimadores dos parâmetros e das estatísticas usadas para verificar o ajuste, também, é muito complicada e usaremos resultados assintóticos. Seguindo o teorema demonstrado por Kaufmann (1987) temos, sob algumas condições de regularidade, que

$$
\sqrt{(T-k)}(\hat{\nu}-\nu) \sim N\left(\mathbf{0}, \boldsymbol{\Omega}(\nu)^{-\mathbf{1}}\right)
$$

em que

$$
\boldsymbol{\Omega}(\nu)=\lim _{T \rightarrow \infty} \frac{4 d_{g} \hat{\phi}^{-1} M^{(\lambda) T} H^{2} M^{(\lambda)}}{(T-k)} \text { e } \hat{\phi}=\frac{\left(y^{(\lambda)}-\mu\right)^{T} D^{(\lambda)}\left(y^{(\lambda)}-\mu\right)}{(T-k)}
$$

para os modelos TSARMA.

A inferência do vetor paramétrico $(\nu, \phi)$ no modelo TSARMA é feita condicionando o parâmetro de transformação $\lambda=\hat{\lambda}$. Dessa forma, a estimativa $\hat{\lambda}$ é vista como o valor verdadeiro, intervalos de confiança e testes de hipóteses para $(\nu, \phi)$ podem ser feitos em um contexto similar ao do MLG a partir do valor estimado $(\hat{\nu}, \hat{\phi})$. Para fazer inferência sobre o parâmetro de transformação, $\lambda$, podemos obter de (2.6) e (2.7) a estatística da razão de verossimilhanças $w=2\left\{l_{p}(\lambda)-l_{p}\left(\lambda^{(0)}\right)\right\}$ para testar $\lambda=\lambda^{(0)}$, a qual tem distribuição assintótica $\chi_{1}^{2}$. E mais, invertendo o teste da razão de verossimilhanças, isto é, $\left\{\lambda \mid l_{p}(\lambda)>l_{p}(\hat{\lambda})-\frac{1}{2} \chi_{1}^{2}(\alpha)\right\}$, podemos construir um intervalo de confiança para $\lambda$, em grandes amostras, pois $\mathbb{P}\left\{w \geq \chi_{1}^{2}(\alpha)\right\}=\alpha+O\left(T^{-1 / 2}\right)$.

\subsection{Diagnóstico de Modelos TSARMA}

O objetivo da análise de diagnóstico não é meramente determinar se há evidências de superparametrização ou subparametrização do modelo, mas também, sugerir formas nas quais o modelo pode ser modificado quando necessário. Para algumas classes gerais de modelos de séries temporais é natural considerar as propriedades estocásticas e a função de autocorrelação dos resíduos e dos resíduos ao quadrado. Esta técnica, por exemplo, é necessária para ajudar a indicar se o modelo capturou de forma significativa a autocorrelação da variável resposta.

Box e Pierce (1970) obtiveram a distribuição da função de autocorrelação residual

$$
\hat{r}_{k}=\frac{\sum_{t=k+1}^{T} \hat{\varepsilon}_{t} \hat{\varepsilon}_{t-k}}{\sum_{t=1}^{T} \hat{\varepsilon}_{t}^{2}}, \quad k=1, \ldots, M
$$

e sugeriram uma estatística portmanteau

$$
Q(M)=n \sum_{k=1}^{M} \hat{r}_{k}^{2}
$$


para testar se os resíduos são ruído branco. Sob a condição de adequacidade do modelo, $Q(M)$ é aproximadamente $\chi_{(M-p-q)}^{2}$ sendo $M$ e $T$ suficientemente grandes e $p$ e $q$ parâmetros do modelo autorregressivo e de médias móveis. Davies, Triggs e Newbold (1977) e Ljung e Box (1978) demonstraram que a estatística modificada

$$
Q^{*}(M)=n(n+2) \sum_{k=1}^{M} \frac{\hat{r}_{k}^{2}}{(n-k)}
$$

está mais próxima da distribuição $\chi^{2}(M-p-q)$ para pequenas amostras.

Seja $\nu=\left(\beta^{T}, \varphi^{T}, \psi^{T}\right)^{T}$, um vetor paramétrico $l \times 1$. Assumiremos que $Z_{t}=Y_{t}^{(\lambda)}$ é invertível. Seja $\varepsilon_{t}=Z_{t}-\mu_{t}(\nu)$ e $\hat{\varepsilon}_{t}$ sendo o resíduo correspondente quando substituímos $\nu$ por $\hat{\nu}$. Similarmente, definiremos $\hat{\mu_{t}}$. Consideraremos o resíduo com $\phi$ conhecido ou fixo.

Seja $r=\left(r_{1}, \ldots, r_{M}\right)^{T}$ e $\hat{r}=\left(\hat{r}_{1}, \ldots, \hat{r}_{M}\right)^{T}$, sendo o vetor de autocorrelações correspondente quando substituímos a $k$-ésima autocovariância residual,

$$
C_{k}=\frac{\sum_{t=k+1}^{T} \varepsilon_{t} \varepsilon_{t-k}}{(T-k)}
$$

pela

$$
\hat{C}_{k}=\frac{\sum_{t=k+1}^{T} \hat{\varepsilon}_{t} \hat{\varepsilon}_{t-k}}{(T-k)}
$$

a $k$-ésima autocovariância residual amostral.

Li (1992) mostrou que, sob algumas condições razoáveis na prática, a distribuição assintótica da autocorrelação residual amostral, $\hat{r}$, é normal multivariada para uma classe geral de modelos de séries temporais não-linear e homoscedástica, a qual contempla os modelos TSARMA. Usando $\mathrm{Li}$ (1992), temos que a distribuição assintótica de $\sqrt{(T-k)} \hat{r}$ é normal com média zero e matriz de covariância $I_{M}-\phi^{-1} J^{T} K_{\nu \nu}^{-1} J$, em que $I_{M}$ é a matriz identidade de ordem $M, J$ é uma matriz $l \times M$ definida como

$$
J=-(T-k)^{-1} \mathrm{E}\left\{\sum_{t=k+1}^{T} \frac{\partial \mu_{t}(\nu)}{\partial \nu} \varepsilon_{t-1}, \ldots, \sum_{t=k+1}^{T} \frac{\partial \mu_{t}(\nu)}{\partial \nu} \varepsilon_{t-M}\right\}
$$

e $K_{\nu \nu}$ é a matriz de informação esperada de Fisher para $\nu$.

Além disso, $Q(M)=(T-k) \hat{r}^{T} \hat{K}_{\nu \nu}^{-1} \hat{r}$ terá distribuição assintótica $\chi^{2}$ com $M$ graus de liberdade se o modelo é correto. Esta quantidade pode ser usada para testar a significância conjunta de $\hat{r}_{k}, \quad k=1, \ldots, M$. 
No entanto, é necessário verificar também se há afastamentos sérios das suposições feitas para o modelo e se existem observações aberrantes. Uma técnica que pode ajudar nesse sentido, é a metodologia de Cox e Snell (1968).

Os resísuos, $\hat{\varepsilon}_{t}=h\left(Z_{t}, \mu(\hat{\nu}), \phi\right)$, em geral, são viesados e não têm distribuição normal, mesmo assintoticamente, dificultando a verificação da adequacidade dos modelos tradicionais.

Cysneiros (2004) corrigiu os dois primeiros momentos de $\hat{\varepsilon}_{t}$ até ordem $n^{-1}$, onde $n$ é o tamanho da amostra, a fim de obter propriedades próximas às do erro, $\varepsilon_{t}=h\left(Z_{t}, \mu_{t}(\nu), \phi\right)$, usando o viés de segunda ordem da estimativa de máxima verossimilhança de $\beta, B(\hat{\beta})$, para modelos de regressão não lineares simétricos, seguindo Cox e Snell (1968). Nós podemos aplicar esse resultado para modelos TSARMA. Vale ressaltar que a expressão para $B(\hat{\beta})$ encontrada em Cysneiros (2004) continua sendo válida para observações dependentes, desde que todos os cumulantes conjuntos das derivadas do logaritmo da função de verossimilhança, $l(\nu, \phi, \lambda)$, com relação a todos os $\nu$ 's sejam $\mathrm{O}(T-k)$, em que $T$ é o tamanho da amostra.

Usando a expansão em série de Taylor de $\hat{\varepsilon}_{t}$ e $\hat{\varepsilon}_{t}^{2}$ em termos de $\hat{\nu}_{i}-\nu_{i}, i=1, \ldots, l$, até ordem $(T-k)^{-1}$, e seguindo Cysneiros (2004), quando os momentos existem, então

$$
\hat{\varepsilon}_{t}=\varepsilon_{t}+\left(\hat{\nu}_{i}-\nu_{i}\right) H_{t}^{i}+\frac{1}{2}\left(\hat{\nu}_{i}-\nu_{i}\right)\left(\hat{\nu}_{j}-\nu_{j}\right) H_{t}^{i j}
$$

em que $H_{t}^{i}=\frac{\partial h\left(Z_{t}, \mu_{t}(\nu), \phi\right)}{\partial \nu_{i}}=\frac{\partial}{\partial \nu_{i}}\left(Z_{t}-\mu_{t}(\nu)\right)=-\frac{\partial \mu_{t}(\nu)}{\partial \nu_{i}}$ e $H_{t}^{i j}=\frac{\partial^{2} h\left(Z_{t}, \mu_{t}(\nu), \phi\right)}{\partial \nu_{i} \partial \nu_{j}}=\frac{\partial}{\partial \nu_{j}}\left[-\frac{\partial \mu_{t}(\nu)}{\partial \nu_{i}}\right]=$ $-\frac{\partial^{2} \mu_{t}(\nu)}{\partial \nu_{i} \partial \nu_{j}}$.

Aplicando a esperança na expressão (2.8) e seguindo Cox e Snell (1968), obtemos

$$
\mathrm{E}\left(\hat{\varepsilon}_{t}\right)=\mathrm{E}\left(\varepsilon_{t}\right)+\mathrm{E}\left(\hat{\nu}_{i}-\nu_{i}\right) \mathrm{E}\left(H_{t}^{i}\right)+I^{i j} \mathrm{E}\left(H_{t}^{i} U_{t}^{j}+\frac{1}{2} H_{t}^{i j}\right)
$$

sendo que no lado direito de (2.9) aplica-se a convenção da soma para $i, j=1, \ldots, l, \mathrm{E}\left(\varepsilon_{t}\right)=0$, $U_{t}^{j}$ é a função escore $\frac{\partial l(\nu, \phi, \lambda)}{\partial \nu_{j}}=\frac{v_{t}}{\phi}\left(y_{t}-\mu_{t}\right) \frac{\partial \mu_{t}}{\partial \nu_{j}}, I_{i j}$ é $(i, j)$-ésimo elemento da matriz $K_{\nu \nu}$, $K_{\nu \nu}(i j)=\frac{4 d_{g}}{\phi} \sum_{t=k+1}^{T} \frac{\partial \mu_{t}}{\partial \nu_{i}} \times \frac{\partial \mu_{t}}{\partial \nu_{j}}, I^{i j}$ é o $(i, j)$-ésimo elemento da matriz $K_{\nu \nu}^{-1}, \mathrm{E}\left(H_{t}^{i}\right)=-\frac{\partial \mu_{t}(\nu)}{\partial \nu_{i}}$, $\mathrm{E}\left(H_{t}^{i j}\right)=-\frac{\partial^{2} \mu_{t}(\nu)}{\partial \nu_{i} \partial \nu_{j}}$ e $\mathrm{E}\left(H_{t}^{i} U_{t}^{j}\right)=0, \forall(i, j)$. Além disso, $\mathrm{E}\left(\hat{\nu}_{i}-\nu_{i}\right)=B\left(\hat{\nu}_{i}\right)$ é o viés de ordem $(T-k)^{-1}$, sendo o $i$-ésimo elemento do vetor $\mathrm{E}(\hat{\nu}-\nu)=B(\hat{\nu})=-{\frac{\phi}{8 d_{g}}}_{[}\left[\frac{\partial \mu(\nu)}{\partial \nu} \frac{\partial \mu(\nu)}{\partial \nu}^{-1} \frac{\partial \mu(\nu)}{\partial \nu}^{T} \boldsymbol{z}\right.$, em que $\boldsymbol{z}$ é um vetor $(T-k) \times 1$ com o $t$-ésimo elemento dado por $z_{t}=\operatorname{tr}\left\{\left[\frac{\partial \mu(\nu)}{\partial \nu} \frac{\partial \mu(\nu)}{\partial \nu}^{-1} \frac{\partial^{2} \mu_{t}(\nu)}{\partial \nu \partial \nu^{T}}\right\}\right.$, $t=1, \ldots,(T-k)$.

Assim, o primeiro momento até ordem $(T-k)^{-1}$ é 


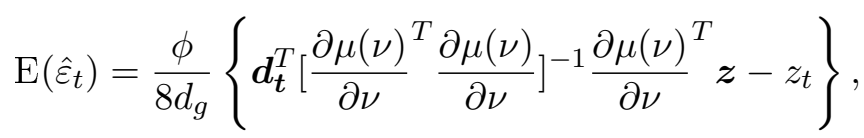

em que $\boldsymbol{d}_{\boldsymbol{t}}=\left(\frac{\partial \mu_{t}}{\partial \nu_{1}}, \ldots, \frac{\partial \mu_{t}}{\partial \nu_{l}}\right)^{T}$, ou consequentemente, em forma matricial

$$
\mathrm{E}(\hat{\varepsilon})=\frac{\phi}{8 d_{g}}\left(\boldsymbol{H}-\boldsymbol{I}_{(T-k)}\right) \boldsymbol{z},
$$

em que $\boldsymbol{H}=\frac{\partial \mu(\nu)}{\partial \nu}\left[\frac{\partial \mu(\nu)}{\partial \nu}{ }^{T} \frac{\partial \mu(\nu)}{\partial \nu}\right]^{-1} \frac{\partial \mu(\nu)}{\partial \nu}^{T}$ e $\boldsymbol{I}_{(T-k)}$ é a matriz identidade de ordem $(T-k)$.

Procedendo de maneira análoga a Cysneiros (2004), podemos encontrar o segundo momento até ordem $(T-k)^{-1}$ como

$$
\mathrm{E}\left(\hat{\varepsilon}_{t}^{2}\right)=\kappa \phi-I^{i j} \frac{\partial \mu_{t}}{\partial \nu_{i}} \frac{\partial \mu_{t}}{\partial \nu_{j}}
$$

e então

$$
\begin{gathered}
\operatorname{VAR}\left(\hat{\varepsilon}_{t}\right)=\kappa \phi\left[1-\left(4 d_{g} \kappa\right)^{-1} h_{t t}\right], \\
\operatorname{Cov}\left(\hat{\varepsilon}_{t}, \hat{\varepsilon}_{t-r}\right)=-\phi \kappa\left(4 d_{g} \kappa\right)^{-1} h_{t t-r}, \quad r \neq 0,
\end{gathered}
$$

em que $h_{t t-r}=\boldsymbol{d}_{t}^{T}\left[\frac{\partial \mu(\nu)}{\partial \nu}^{T} \frac{\partial \mu(\nu)}{\partial \nu}\right]^{-1} \boldsymbol{d}_{\boldsymbol{t}-\boldsymbol{r}}$. Em notação matricial ficamos com a matriz de covariâncias do vetor de resíduos ordinários expressa por

$$
\operatorname{VAR}(\hat{\varepsilon})=\phi \kappa\left\{\boldsymbol{I}_{(T-k)}-\left(4 d_{g} \kappa\right)^{-1} \boldsymbol{H}\right\}
$$

\subsection{Estimativa na Escala Original}

Granger e Newbold (1976) observaram que ótimas previsões obtidas de séries temporais transformadas através de transformações potência não conservam suas propriedades quando são trazidas de volta para a sua escala original. Esse fato ocorre, pois uma média estimada de uma distribuição simétrica na escala transformada torna-se uma mediana estimada após uma aplicação da transformação inversa. Muitos autores, como, por exemplo, Neymann e Scott (1960), Miller (1984), Taylor (1986), Pankratz e Dudley (1987) e Guerrero (1993) propuseram métodos para corrigir o viés da estimativa do valor médio na escala original. Uma maneira de 
tratar essa questão é apresentada nesta seção, considerando a transformação de Box-Cox.

Considerando a transformação de Box-Cox, inicialmente, estimaremos a média da variável resposta não transformada $Y_{t}$ usando um método análogo ao método small- $\theta$ apresentado em Draper e Cox (1969) e, em seguida, obteremos o r-ésimo momento ordinário de $Y_{t}$. Nesse sentido, estamos utilizando a ideia apresentada em Cordeiro e Andrade (2009). Quando $\lambda \neq 0$

$$
Y_{t}=\left(1+\lambda \mu_{t}\right)^{\frac{1}{\lambda}}\left\{1+\xi_{t}\left(Y_{t}^{(\lambda)}-\mu_{t}\right)\right\}^{\frac{1}{\lambda}}
$$

em que $\xi_{t}=\frac{\lambda}{1+\lambda \mu_{t}}$. A partir da expansão binomial, obtemos

$$
\left\{1+\xi_{t}\left(Y_{t}^{(\lambda)}-\mu_{t}\right)\right\}^{\frac{1}{\lambda}}=1+\sum_{i=1}^{\infty} \frac{\xi^{i}}{i !} \prod_{j=0}^{i-1}\left(\frac{1}{\lambda}-j\right)\left(Y_{t}^{(\lambda)}-\mu_{t}\right)^{i}
$$

Como os momentos centrais ímpares são nulos, então temos

$$
\mathrm{E}\left(Y_{t} \mid H_{t}\right)=\left(1+\lambda \mu_{t}\right)^{\frac{1}{\lambda}}\left\{1+\sum_{i=1}^{\infty} \frac{a_{2 i} \mu_{t 2 i}}{(2 i) !\left(1+\lambda \mu_{t}\right)^{2 i}}\right\},
$$

em que $a_{2 i}=\prod_{j=0}^{2 i-1}(1-j \lambda)$ e $\mu_{t 2 i}$ é o $(2 i)$-ésimo momento central condicional de $Y_{t}^{(\lambda)}$. Os momentos centrais pares das distribuições simétricas são facilmente obtidos das suas respectivas funções características.

A equação (2.11) generaliza a expansão dada por Pankratz e Dudley (1987) para o fator não linear obtido quando $\lambda^{-1}$ é um inteiro positivo e os dados transformados têm distribuição normal. Se considerarmos somente o primeiro termo da equação (2.11), obtemos a seguinte aproximação

$$
\mathrm{E}\left(Y_{t} \mid H_{t}\right)=\left(1+\lambda \mu_{t}\right)^{\frac{1}{\lambda}}\left\{1+\frac{(1-\lambda) \kappa \phi}{2\left(1+\lambda \mu_{t}\right)^{2}}\right\}
$$

A equação (2.12) é uma generalização da expressão apresentada em Taylor (1986) e Guerrero (1993), as quais são válidas somente para dados normais. O fator corrigido entre chaves é maior que um se $\lambda<1$ e menor que um se $\lambda>1$.

Além disso, podemos obter o $r$-ésimo momento de $Y_{t}$ por expansão binomial de $\left\{1+\xi_{t}\left(Y_{t}^{(\lambda)}-\right.\right.$ $\left.\left.\mu_{t}\right)\right\}^{\frac{r}{\lambda}}$ da mesma forma. Assim,

$$
\mathrm{E}\left(Y_{t}^{r} \mid H_{t}\right)=\left(1+\lambda \mu_{t}\right)^{\frac{r}{\lambda}}\left\{1+\sum_{i=1}^{\infty} \frac{b_{2 i}^{(r)} \mu_{t 2 i}}{(2 i) !\left(1+\lambda \mu_{t}\right)^{2 i}}\right\},
$$


em que $b_{2 i}^{(r)}=\prod_{j=0}^{2 i-1}(r-j \lambda)$. Claramente, $b_{2 i}^{(1)}=a_{2 i}$. Combinando (2.11) e (2.13), podemos obter todos os momentos de $Y_{t}$, até qualquer ordem de $\left(1+\lambda \mu_{t}\right)^{-k}$ para $k \geq 2$. Em especial, a variância de $Y_{t}$ para ordem $\left(1+\lambda \mu_{t}\right)^{-4}$ é expressa como

$$
\operatorname{VAR}\left(Y_{t} \mid H_{t}\right)=\left(1+\lambda \mu_{t}\right)^{\frac{2}{\lambda}}\left[\frac{\mu_{t 2}}{\left(1+\lambda \mu_{t}\right)^{2}}+\frac{(1-\lambda)\left\{(7-11 \lambda) \mu_{t 4}-3(1-\lambda) \mu_{t 2}^{2}\right\}}{12\left(1+\lambda \mu_{t}\right)^{4}}\right]
$$

Uma estimativa óbvia de $\mathrm{E}\left(Y_{t}^{r} \mid H_{t}\right)$ segue, usando as estimativas dos parâmetros $\lambda$, $\mu_{t}$ e $\phi$. Quando $\lambda=0$, podemos obter de $\mathrm{E}\left(Y_{t}^{r} \mid H_{t}\right)=e^{r \mu_{t}} \mathrm{E}\left(e^{r\left(Y_{t}^{(0)}-\mu_{t}\right)} \mid H_{t}\right)$

$$
\mathrm{E}\left(Y_{t}^{r} \mid H_{t}\right)=e^{r \mu_{t}} \mathrm{E}\left(1+\sum_{i=1}^{\infty} \frac{r^{2 i} \mu_{t 2 i}}{(2 i) !}\right) .
$$

Então, a variância de $Y_{t}$ é

$$
\operatorname{VAR}\left(Y_{t} \mid H_{t}\right)=e^{2 \mu_{t}}\left[\sum_{i=1}^{\infty}\left\{\frac{\left(2^{2 i}-2\right) \mu_{t 2 i}}{(2 i) !}+\frac{\mu_{t 2 i}^{2}}{(2 i) !^{2}}\right\}-2 \sum_{i \neq j=1}^{\infty} \frac{\mu_{t 2 i} \mu_{t 2 j}}{(2 i) !(2 j) !}\right]
$$

Para uma transformação geral $(1.1)$, seja $Y_{t}=F\left(Y_{t}^{(\lambda)}, \lambda\right)$ sua inversa. Expandindo $F$ por série de Taylor, temos

$$
\mathrm{E}\left(Y_{t} \mid H_{t}\right)=F\left(\mu_{t}, \lambda\right)+\sum_{i=1}^{\infty} \frac{F^{(2 i)}\left(\mu_{t}, \lambda\right) \mu_{t 2 i}}{(2 i) !}
$$

em que $F^{(2 i)}\left(\mu_{t}, \lambda\right)$ é a $(2 i)$-ésima derivada de $F\left(\mu_{t}, \lambda\right)$ com respeito a $\mu_{t}$. Analogamente, o $r$-ésimo momento de $Y_{t}$ é expresso como

$$
\mathrm{E}\left(Y_{t}^{r} \mid H_{t}\right)=F\left(\mu_{t}, \lambda\right)^{r}+\sum_{i=1}^{\infty} \frac{G^{(2 i)}\left(\mu_{t}, \lambda\right) \mu_{t 2 i}}{(2 i) !},
$$

em que $G^{(2 i)}\left(\mu_{t}, \lambda\right)$ é a $(2 i)$-ésima derivada de $G\left(\mu_{t}, \lambda\right)=F\left(\mu_{t}, \lambda\right)^{r}$ com respeito a $\mu_{t}$.

\subsection{Simulações}

Nesta seção, temos como principal objetivo avaliar a performance do modelo TSARMA, no que diz respeito ao comportamento das estimativas dos parâmetros. Dessa forma, realizamos algumas simulações, no programa computacional R, para observar o comportamento das estimativas mediante situações específicas, avaliadas de acordo com o viés e o erro quadrático médio, EQM. Para todos os modelos simulados fixamos o número de réplicas em 1.000, utilizamos 
amostras de tamanho $T=250$ e $T=500$. Quanto aos modelos transformados, a transformação usada foi a de Box-Cox. Estimamos o parâmetro de transformação, $\lambda$, maximizando numericamente o logaritmo da função de verossimilhança perfilada por meio do gráfico de $l_{p}(\lambda)$ versus $\lambda$, usando uma grade de valores para $\lambda$ que variava entre 0,00 a 2,00 de $0,01 \mathrm{em} 0,01$.

Inicialmente, geramos dados para o modelo (2.14), ou seja, um conjunto de dados cuja distribuição condicional é normal, levamos em consideração uma variável explicativa gerada a partir de um modelo $\mathrm{AR}(1)$ com parâmetro autorregressivo igual 0,7, equação (2.16), e para o componente sistemático, usamos a função identidade como função de ligação, expressão (2.15).

$$
y_{t}^{(1)}=\mu_{t}+\sqrt{2} \varepsilon_{t}, \quad \varepsilon_{t} \sim N(0,1)
$$

satisfazendo

$$
\mu_{t}=\eta_{t}
$$

em que

$$
\begin{aligned}
\eta_{t}=50+ & 3 x_{t}+0,7\left(y_{t-1}-50-3 x_{t-1}\right)+0,2\left(y_{t-1}-\eta_{t-1}\right), \\
x_{t} & =0,7 x_{t-1}+\epsilon_{t}, \quad \epsilon_{t} \sim \text { i.i.d. } N(0,1), \\
t & =1, \ldots, T .
\end{aligned}
$$

\begin{tabular}{crrrrrr}
\hline$T$ & \multicolumn{1}{c}{$\hat{\lambda}$} & \multicolumn{1}{c}{$\hat{\beta}_{0}$} & \multicolumn{1}{c}{$\hat{\beta}_{1}$} & \multicolumn{1}{c}{$\hat{\varphi}$} & $\hat{\psi}$ & \multicolumn{1}{c}{$\hat{\phi}$} \\
\hline & & & & & & \\
250 & 0,9972 & 49,9513 & 3,0107 & 0,7563 & 0,0168 & 2,0771 \\
Viés & 0,0028 & 0,0487 & $-0,0107$ & $-0,0563$ & 0,1832 & $-0,0771$ \\
EQM & 0,0017 & 33,2870 & 0,2481 & 0,0048 & 0,0342 & 0,4188 \\
& & & & & & \\
500 & 1,0007 & 50,4400 & 3,0470 & 0,7542 & 0,0371 & 2,1341 \\
Viés & $-0,0006$ & $-0,4400$ & $-0,0470$ & $-0,0542$ & 0,1629 & $-0,1341$ \\
EQM & 0,0017 & 33,9848 & 0,2354 & 0,0038 & 0,0273 & 0,4136
\end{tabular}

Tabela 2.3: Valores para os EMV dos respectivos parâmetros $\lambda=1, \beta_{0}=50, \beta_{1}=3, \varphi=0,7, \psi=$ 0,2 e $\phi=2$ para distribuição normal

Na Tabela 2.3, podemos observar o desempenho das estimativas com os seus respectivos vieses e erros quadrático médio, EQM, para o modelo transformado (2.14). Vemos que não 
houve grande diferença entre os valores estimados e seus respectivos vieses e EQM quando aumentamos o tamanho da amostra de 250 para 500, e que os valores do EQM são grandes para a estimativa de $\beta_{0}$. A Figura 2.1 apresenta os histogramas e os QQ-plots construídos com as estimativas dos parâmetros $\lambda, \beta_{1}, \varphi$ e $\phi$, a partir das 1000 réplicas, usando o modelo (2.14) com $T=500$. Percebemos que apenas a distribuição do estimador de $\varphi$ tende a ser normal.

\begin{tabular}{crrrr}
\hline$T$ & $\hat{\beta}_{1}$ & \multicolumn{1}{c}{$\hat{\varphi}$} & $\hat{\psi}$ & $\hat{\phi}$ \\
\hline & & & & \\
250 & 2,9970 & 0,7034 & 0,1975 & 1,9756 \\
Viés & 0,0030 & $-0,0034$ & 0,0025 & 0,0244 \\
EQM & 0,0187 & 0,0030 & 0,0052 & 0,0317 \\
& & & & \\
500 & 2,9942 & 0,6985 & 0,2100 & 1,9963 \\
Viés & 0,0058 & 0,0015 & $-0,0100$ & 0,0037 \\
EQM & 0,0067 & 0,0013 & 0,0033 & 0,0158
\end{tabular}

Tabela 2.4: Valores para os EMV dos respectivos parâmetros $\beta_{1}=3, \varphi=0,7, \psi=0,2$ e $\phi=2$ para distribuição normal.
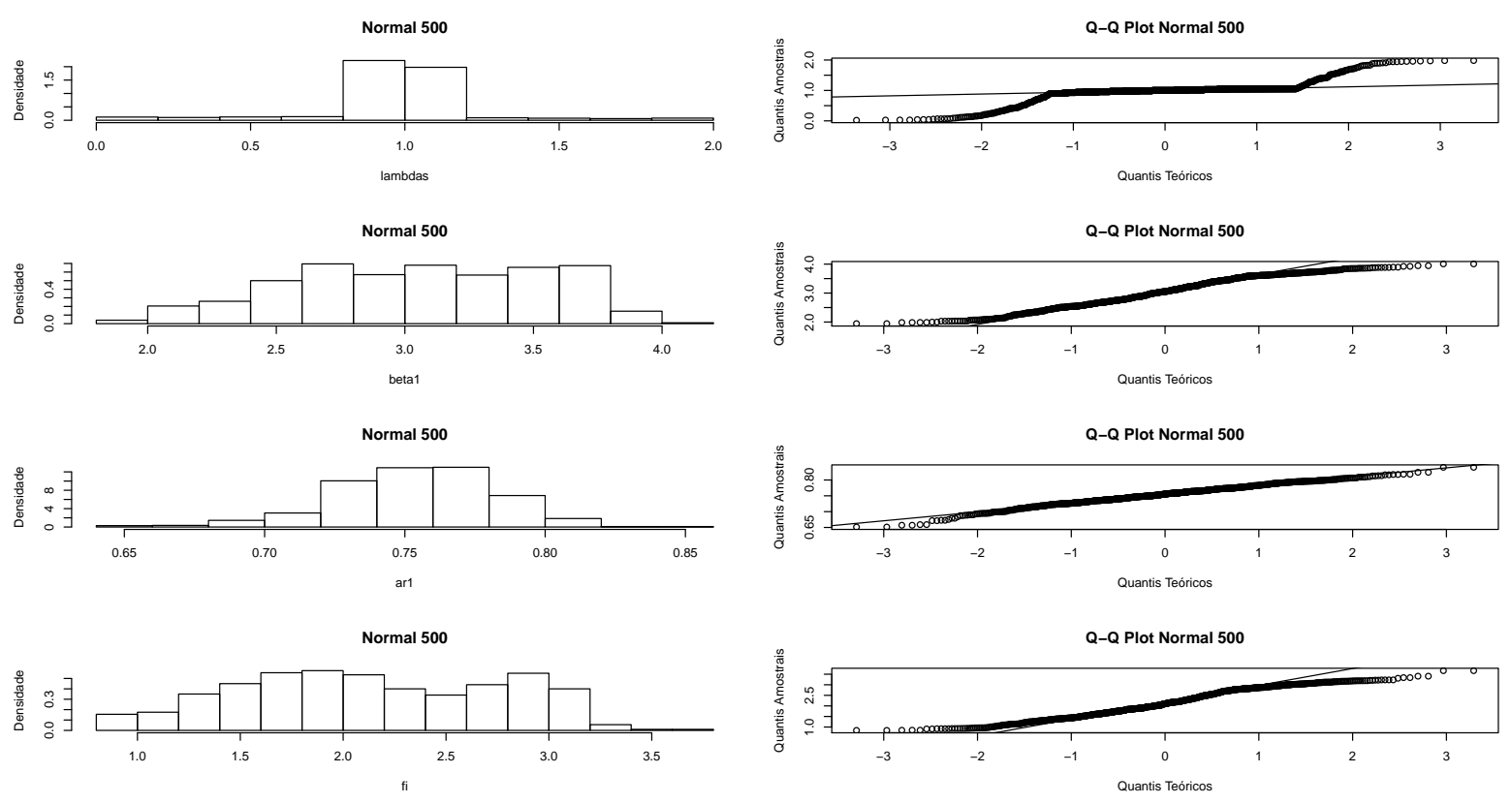

Figura 2.1: Histogramas e QQ-plot para os estimadores de $\lambda, \beta_{1}, \varphi$ e $\phi$, respectivamente, do modelo normal com transformação.

Para o modelo (2.17) não estimamos o parâmetro de transformação, e utilizamos o fato de que $\lambda=1$, observe também que não utilizamos o parâmetro $\beta_{0}$, visto que agora não há necessidade das observações serem positivas e a fim de tornar as simulações mais rápidas. Os resultados das simulações referente ao modelo (2.17) encontram-se na Tabela 2.4, podemos observar que houve 

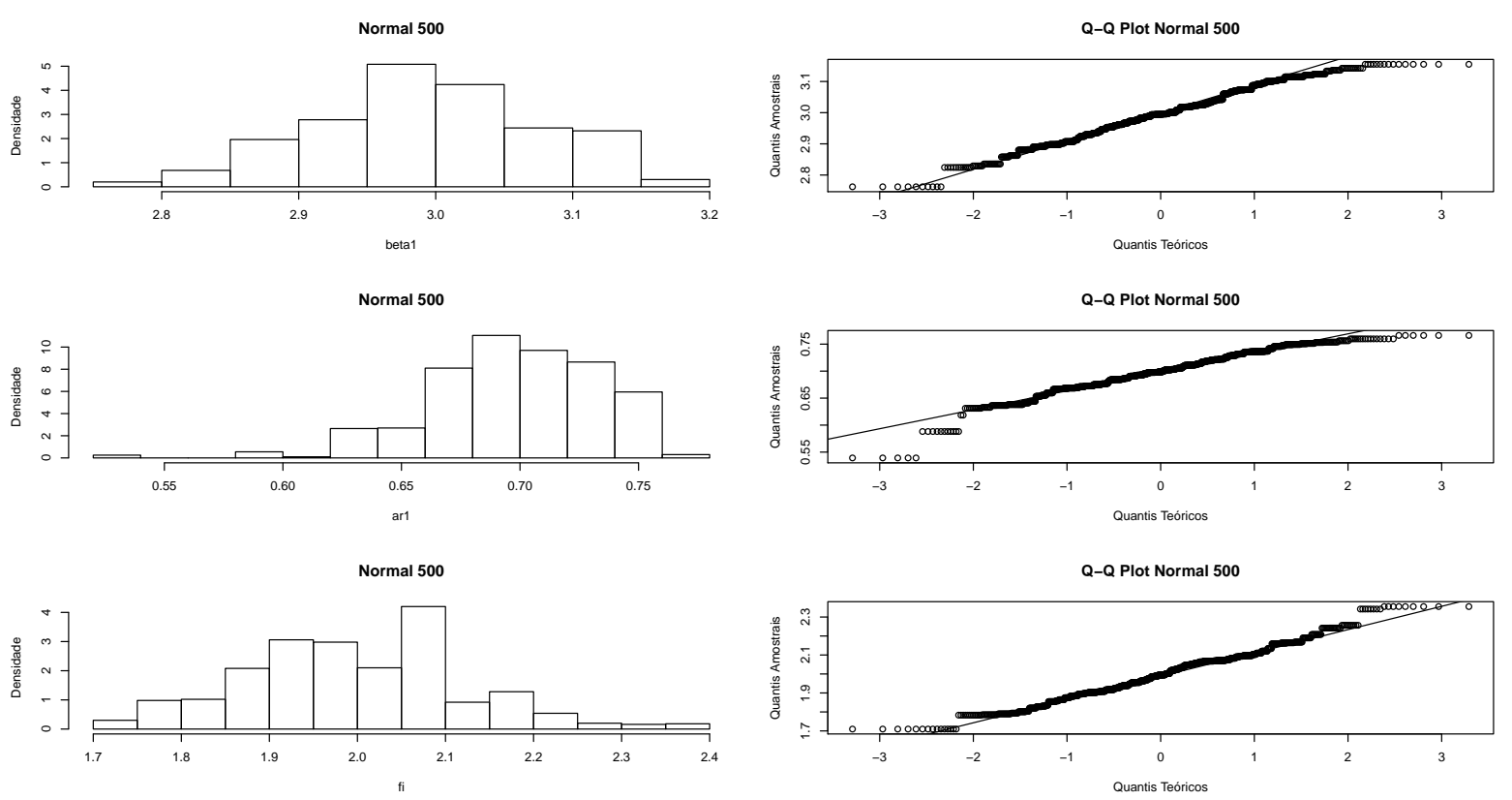

Figura 2.2: Histogramas e QQ-plot para os estimadores de $\beta_{1}, \varphi$ e $\phi$, respectivamente, do modelo normal sem transformação.

melhor precisão nas estimativas dos parâmetros para o modelo (2.17), ou seja, o modelo sem o parâmetro de transformação, $\lambda$, possui estimativas em média com menor viés e EQM. Contudo, as estimativas do modelo transformado, também, possuem vieses e EQM aceitáveis. Um estudo mais detalhado deverá ser realizado a fim de investigar o comportamento dos vieses e EQM para os parâmetros $\hat{\beta}_{0}$ e $\hat{\beta}_{1}$ dados na Tabela 2.3. A Figura 2.2 mostra os histogramas e os QQ-plots das estimativas para o modelo (2.17), construída da mesma forma que a Figura 2.1. Notamos que as distribuições dos estimadores não tendem à normal, possuem um comportamento parecido com uma distribuição de caudas mais pesadas que a distribuição normal.

$$
y_{t}=3 x_{t}+0,7\left(y_{t-1}-50-3 x_{t-1}\right)+0,2\left(y_{t-1}-\eta_{t-1}\right)+\sqrt{2} \varepsilon_{t}, \quad \varepsilon_{t} \sim N(0,1) .
$$

A Tabela 2.5 apresenta os resultados para um modelo similar ao modelo (2.17), porém a distribuição usada é logística II. Vemos que o desempenho das estimativas é semelhante ao desempenho das estimativas do modelo (2.17). Na Tabela 2.6, temos os resultados para um modelo semelhante ao modelo (2.14) com distribuição logística II. Podemos observar que as estimativas do modelo (2.14) têm uma performance melhor do que as estimativas do modelo similar com distribuição logística II. As Figuras 2.3 e 2.4 exibem os histogramas e os QQ-plots para as estimativas dos modelos com distribuição logística II com transformação e sem transformação, 


\begin{tabular}{crrrr}
\hline$T$ & $\hat{\beta_{1}}$ & $\hat{\varphi}$ & $\hat{\psi}$ & $\hat{\phi}$ \\
\hline & & & & \\
250 & 2,9961 & 0,6813 & 0,2065 & 1,9925 \\
Viés & 0,0039 & 0,0187 & $-0,0065$ & 0,0075 \\
EQM & 0,0740 & 0,0039 & 0,0065 & 0,0447 \\
& & & & \\
500 & 2,9958 & 0,6902 & 0,2048 & 1,9940 \\
Viés & 0,0042 & 0,0098 & $-0,0048$ & 0,0060 \\
EQM & 0,0355 & 0,0018 & 0,0028 & 0,0210 \\
& & & &
\end{tabular}

Tabela 2.5: Valores para os EMV dos respectivos parâmetros $\beta_{1}=3, \varphi=0,7, \psi=0,2$ e $\phi=2$ para distribuição logística II.

respectivamente. Percebemos que pequenas variações das estimativas do parâmetro de transformação em relação ao seu valor verdadeiro, $\lambda=1$, conduzem a grandes variações nas estimativas dos outros parâmetros do modelo em relação aos seus respectivos valores verdadeiros, salvo as estimativas do parâmetro autorregressivo, $\varphi=0,7$ que teve pequenas variações em relação ao valor populacional. Essa observação torna-se mais visível quando analisamos a Figura 2.4, pois agora sem estimar o parâmetro de transformação as estimativas variam menos em relação ao valor verdadeiro e as distribuições tendem à distribuição normal mais rapidamente.
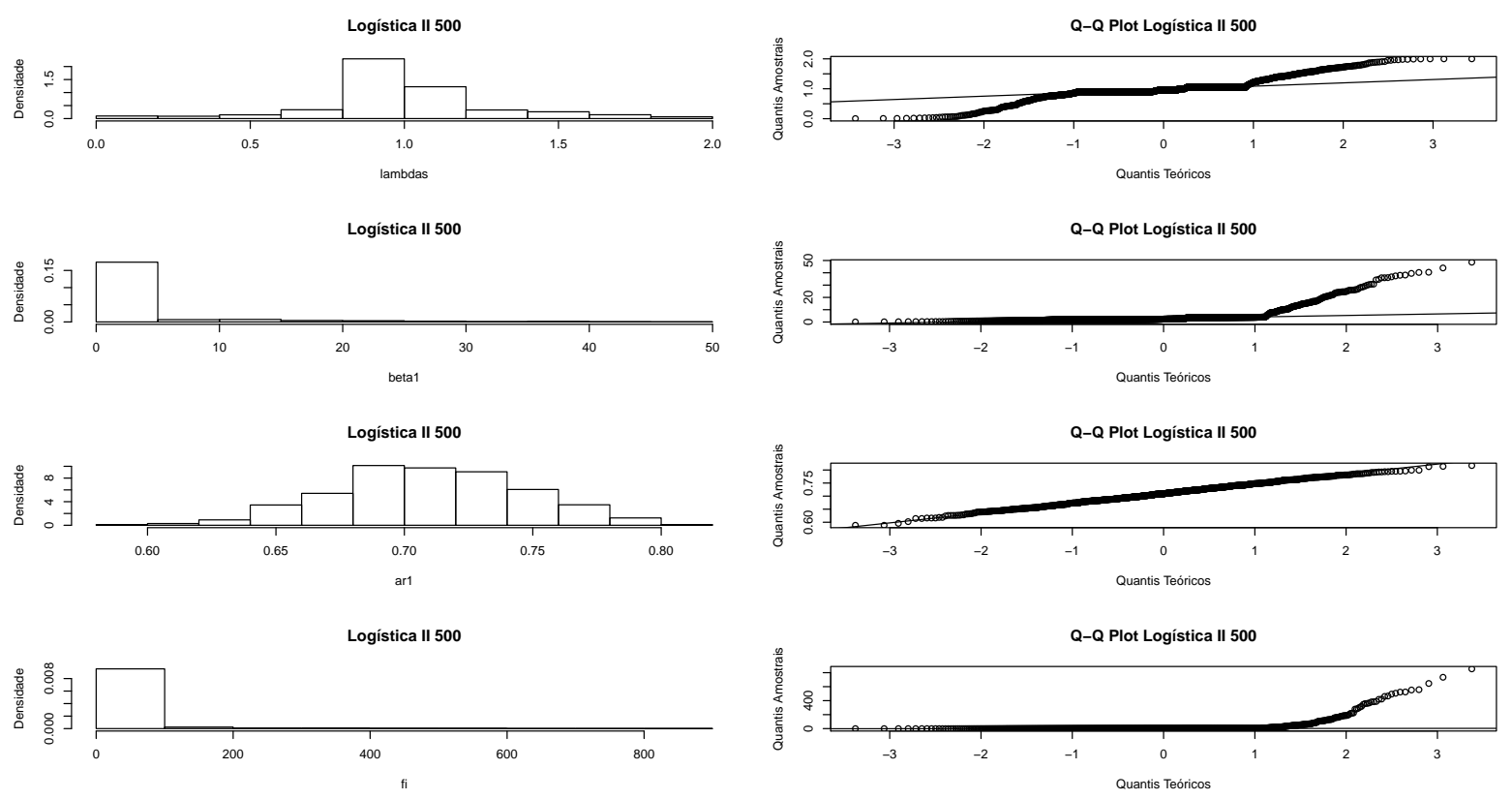

Figura 2.3: Histogramas e QQ-plot para os estimadores de $\lambda, \beta_{1}, \varphi$ e $\phi$, respectivamente, do modelo logístico com transformação.

Estudos similares aos realizados com a distribuição normal e logística foram realizados com a distribuição t-Student com 5 graus de liberdade. As Tabelas 2.7 e 2.8 apresentam esses 


\begin{tabular}{cccrrrc}
\hline$T$ & $\hat{\lambda}$ & $\hat{\beta}_{0}$ & $\hat{\beta}_{1}$ & $\hat{\varphi}$ & $\hat{\psi}$ & $\hat{\phi}$ \\
\hline & & & & & & \\
250 & 0,9372 & 42,5351 & 2,4233 & 0,7195 & 0,1144 & 1,7098 \\
Viés & 0,0628 & 7,4649 & 0,5767 & $-0,0195$ & 0,0856 & 0,2902 \\
EQM & 0,0078 & 126,5462 & 0,7818 & 0,0027 & 0,0108 & 0,2718 \\
& & & & & & \\
500 & 0,9622 & 45,8319 & 2,6794 & 0,7057 & 0,1675 & 1,8685 \\
Viés & 0,0378 & 4,1681 & 0,3206 & $-0,0057$ & 0,0325 & 0,1315 \\
EQM & 0,0061 & 104,2022 & 0,6610 & 0,0013 & 0,0034 & 0,2461 \\
& & & & & & \\
\hline
\end{tabular}

Tabela 2.6: Valores para os EMV dos respectivos parâmetros $\lambda=1, \beta_{0}=50, \beta_{1}=3, \varphi=0,7, \psi=$ 0,2 e $\phi=2$ para distribuição logística II.

\begin{tabular}{crrrr}
\hline$T$ & \multicolumn{1}{c}{$\hat{\beta}_{1}$} & $\hat{\varphi}$ & $\hat{\psi}$ & $\hat{\phi}$ \\
\hline & & & & \\
250 & 3,0008 & 0,6965 & 0,2011 & 1,9991 \\
Viés & $-0,0008$ & 0,0035 & $-0,0011$ & 0,0009 \\
EQM & 0,0127 & 0,0013 & 0,0025 & 0,0266 \\
& & & & \\
500 & 2,9958 & 0,6902 & 0,2048 & 1,9940 \\
Viés & 0,0042 & 0,0098 & $-0,0048$ & 0,0060 \\
EQM & 0,0355 & 0,0018 & 0,0028 & 0,0210 \\
& & & &
\end{tabular}

Tabela 2.7: Valores para os EMV dos respectivos parâmetros $\beta_{1}=3, \varphi=0,7, \psi=0,2$ e $\phi=2$ para distribuição t-Student(5).

resultados. A partir dessas tabelas, podemos observar que as estimativas se comportam de forma parecida aos casos anteriores, com relação aos valores dos vieses e dos EQM, inclusive quanto ao fato de que o EQM é grande para a estimativa de $\beta_{0}$. Porém, verificamos que as estimativas dos modelos com a distribuição t-Student(5) convergem mais lentamente, pois a distribuição t-Student(5) possui caudas mais pesadas do que as demais distribuições utilizadas, isto é, a normal e a logística II. Também, foram realizadas simulações com as distribuições t-Student com 10, 20 e 30 graus de liberdade. O desempenho das estimativas foi como esperado, ou seja, à medida que o número de graus de liberdade foi crescendo, os vieses e os erros quadráticos médios foram diminuindo. Nas Figuras 2.5 e 2.6, podemos verificar que os comportamentos para os modelos t-Student(5) com transformação e sem transformação são parecidos aos comportamentos dos respectivos modelos similares estudados, isto é, há grandes variações nas estimativas dos parâmetros dos modelos transformados, enquanto que, não há grandes variações nas estimativas para os parâmetros dos modelos sem transformação.

Ainda, com o objetivo de avaliar a capacidade preditiva do modelo TSARMA, geramos 


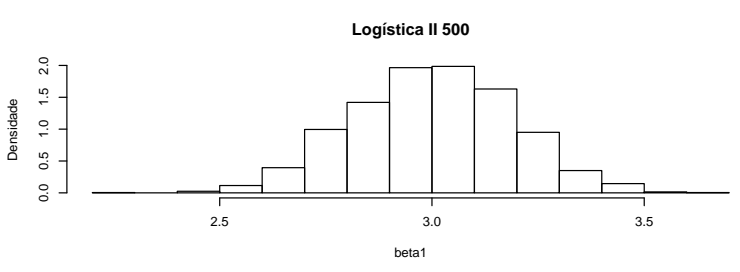

ogistica II 500

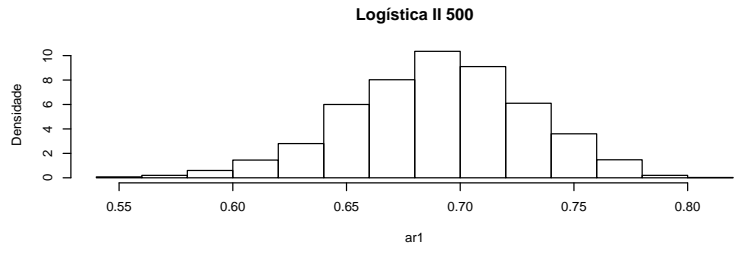

Logistica II 500

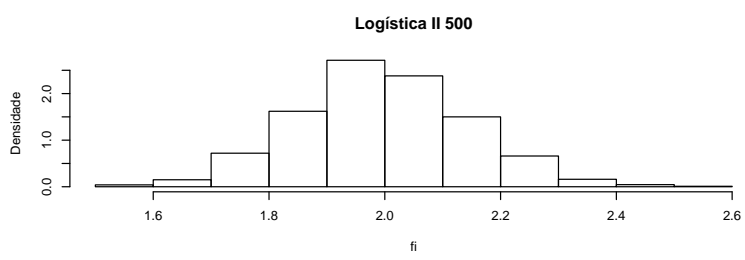

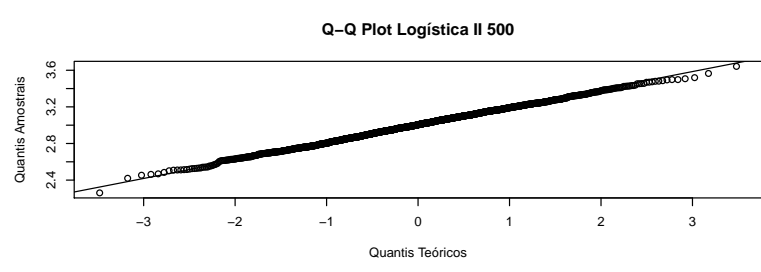

Q-Q Plot Logistica II 500

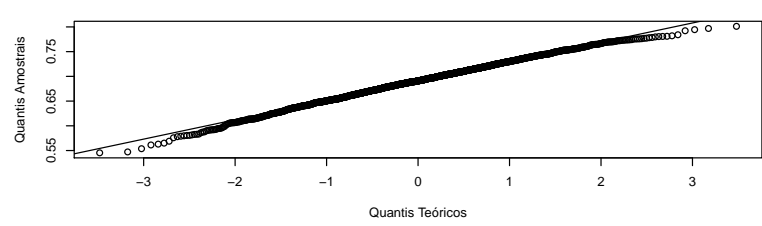

Q-Q Plot Logistica II 500

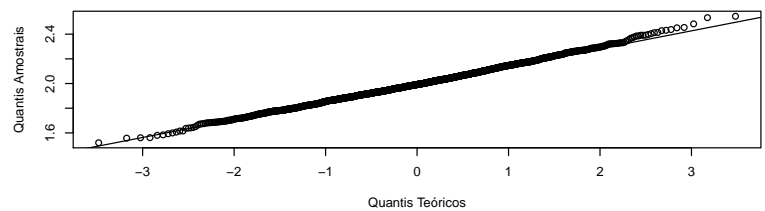

Figura 2.4: Histogramas e QQ-plot para os estimadores de $\beta_{1}, \varphi$ e $\phi$, respectivamente, do modelo logístico sem transformação.

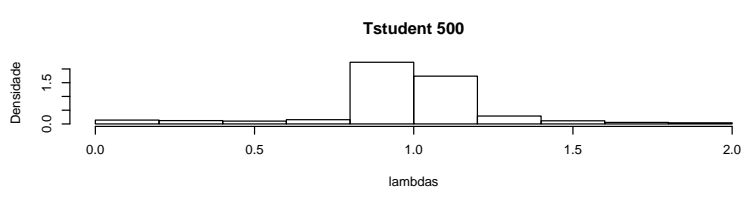

Tstudent 500

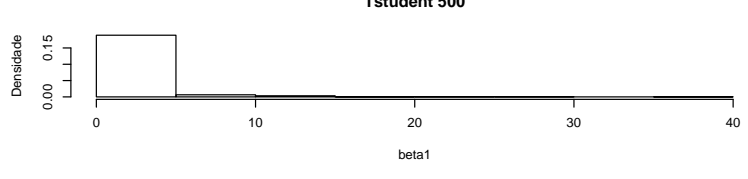

Tstudent 500

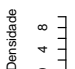

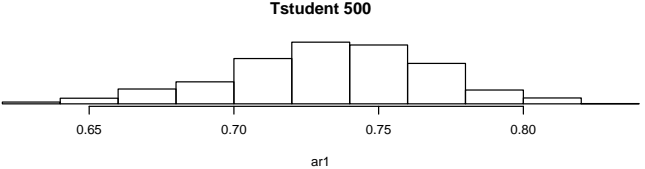

Tstudent 500

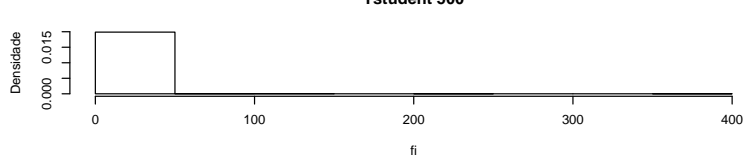

Q-Q Plot Tstudent 500

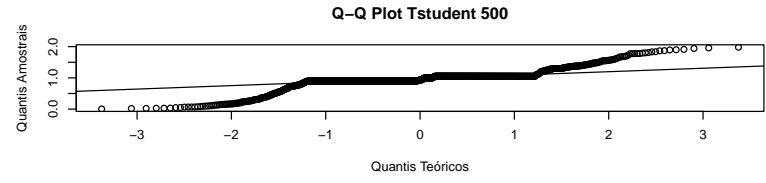

Q-Q Plot Tstudent 500

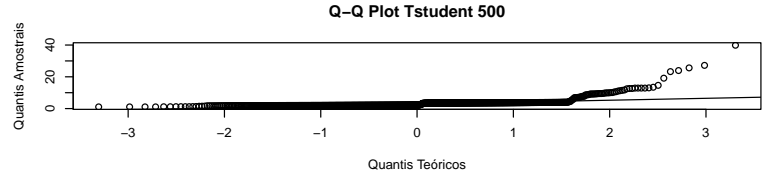

Q-Q Plot Tstudent 500

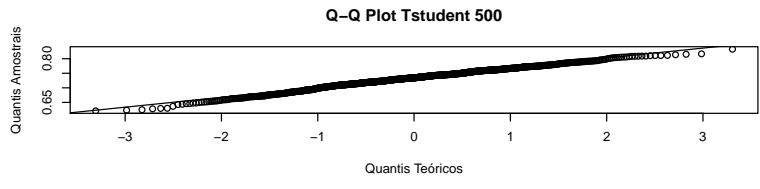

Q-Q Plot Tstudent 500

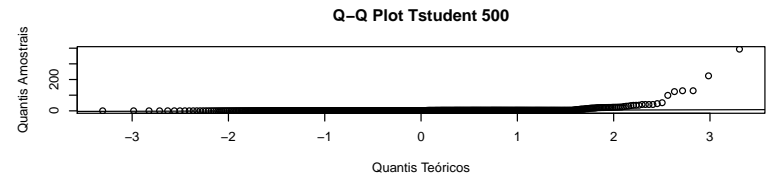

Figura 2.5: Histogramas e QQ-plot para os estimadores de $\lambda, \beta_{1}, \varphi$ e $\phi$, respectivamente, do modelo t-Student(5) com transformação.

uma amostra com 1000 observações, supondo que a distribuição condicional é t-Student com 5 graus de liberdade, levando em consideração uma variável explicativa gerada a partir de um modelo AR(1) com parâmetro autorregressivo igual a 0,9 e distribuição t-Student com 5 graus de liberdade com média zero e variância 3. A função identidade como função de ligação para o componente sistemático, equação (2.18) satisfazendo (2.19) em que (2.20). Então, vamos 


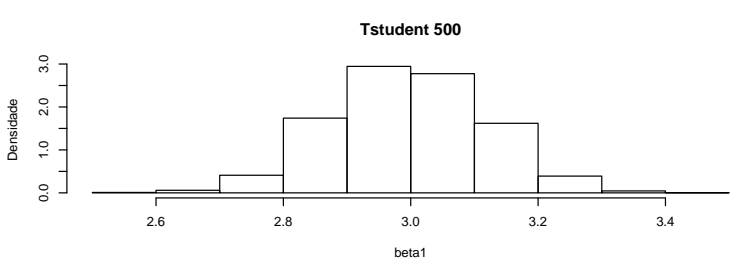

Tstudent 500

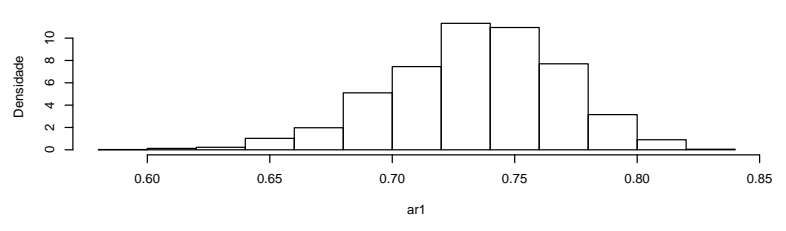

Tstudent 500

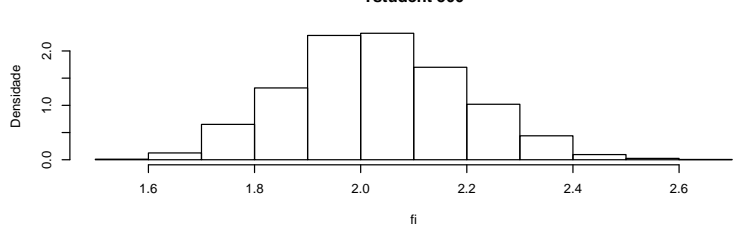

Q-Q Plot Tstudent 500

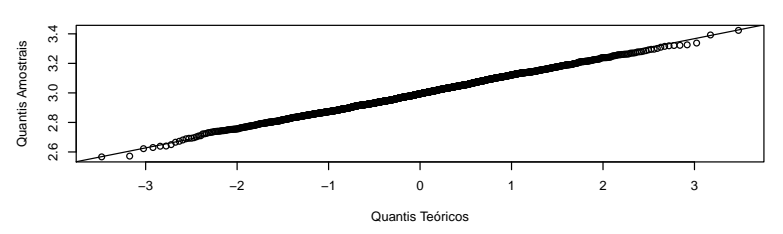

Q-Q Plot Tstudent 500

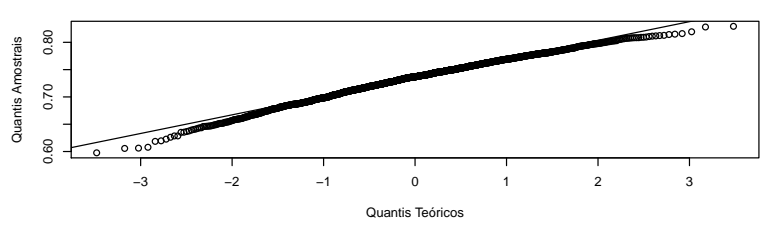

Q-Q Plot Tstudent 500

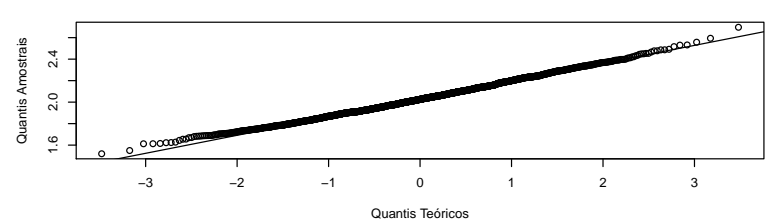

Figura 2.6: Histogramas e QQ-plot para os estimadores de $\beta_{1}, \varphi$ e $\phi$, respectivamente, do modelo tStudent(5) sem transformação.

\begin{tabular}{crcrrrr}
\hline$T$ & $\hat{\lambda}$ & \multicolumn{1}{c}{$\hat{\beta}_{0}$} & $\hat{\beta}_{1}$ & $\hat{\varphi}$ & $\hat{\psi}$ & $\hat{\phi}$ \\
\hline \multirow{2}{*}{250} & 1,1004 & 76,1072 & 5,3303 & 0,7513 & 0,0351 & 8,8620 \\
Viés & $-0,1004$ & $-26,1072$ & $-2,3303$ & $-0,0513$ & 0,1649 & $-6,8620$ \\
EQM & 0,0345 & 1994,6380 & 15,6793 & 0,0042 & 0,0295 & 130,0835 \\
& & & & & & \\
500 & 0,9785 & 49,0197 & 2,9610 & 0,7327 & 0,0979 & 2,3878 \\
Viés & 0,0215 & 0,9803 & 0,0390 & $-0,0327$ & 0,1021 & $-0,3878$ \\
EQM & 0,0086 & 248,2624 & 1,7694 & 0,0023 & 0,0139 & 11,2118 \\
& & & & & & \\
\hline
\end{tabular}

Tabela 2.8: Valores para os EMV dos respectivos parâmetros $\lambda=1, \beta_{0}=50, \beta_{1}=3, \varphi=0,7, \psi=$ 0,2 e $\phi=2$ para distribuição t-Student(5).

modelar essa amostra com o modelo ARMA usual e com o modelo TSARMA segundo várias distribuições simétricas e, assim, comparar essas modelagens.

$$
y_{t}^{(1)}=\mu_{t}+\sqrt{3} \varepsilon_{t}, \quad \varepsilon_{t} \sim t(5),
$$

satisfazendo

$$
\mu_{t}=\eta_{t},
$$




$$
\begin{gathered}
\eta_{t}=200+5 x_{t}+0,3\left(y_{t-1}-200-5 x_{t-1}\right)+0,6\left(y_{t-1}-\eta_{t-1}\right), \\
x_{t}=0,9 x_{t-1}+\epsilon_{t}, \quad \epsilon_{t} \sim \text { i.i.d.t }(5), \\
t=1, \ldots, T .
\end{gathered}
$$
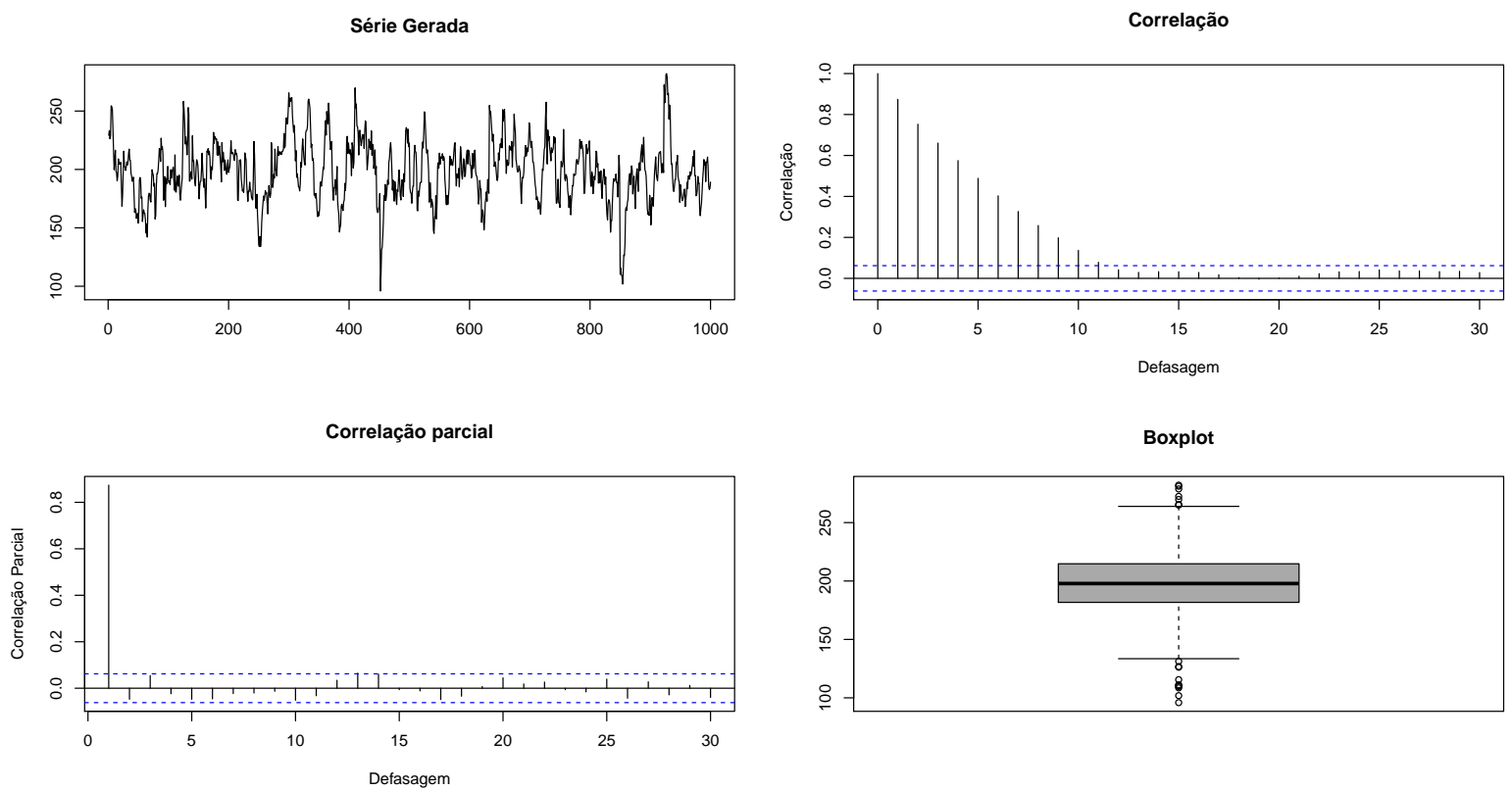

Figura 2.7: Gráficos da série, da função de autocorrelação, da função de autocorrelação parcial e box-plot para o conjunto de observações gerados a partir do modelo (2.18).

A Figura 2.7 fornece os gráficos da série, da função de autocorrelação, da função de autocorrelação parcial e box-plot para o conjunto de 1000 observações gerados a partir do modelo (2.18). Temos indícios, do gráfico da série, que os dados não possuem tendência nem sazonalidade, o box-plot sugere que a série é simétrica com caudas pesadas e os gráficos de autocorrelação e autocorrelação parcial indicam que, de acordo, com a modelagem Box-Jenkins, um modelo adequado seria o $\operatorname{AR}(1)$. Então, inicialmente, estimamos um modelo $\operatorname{AR}(1)$ que fica como na expressão (2.21) e sua análise residual pode ser vista na Figura 2.8 que exibe que o modelo AR(1) se ajusta bem à série gerada.

$$
\begin{array}{r}
\hat{Y}_{t}=\begin{array}{c}
198,3708 \\
{[0,0153]}
\end{array} \quad 0,8745 Y_{t-1}, \\
{[3,1224]}
\end{array}
$$

em que $\hat{\sigma}_{w}^{2}=155,7757$ é a variância estimada dos resíduos e os valores entre colchetes são os respectivos desvios padrões das estimativas. 

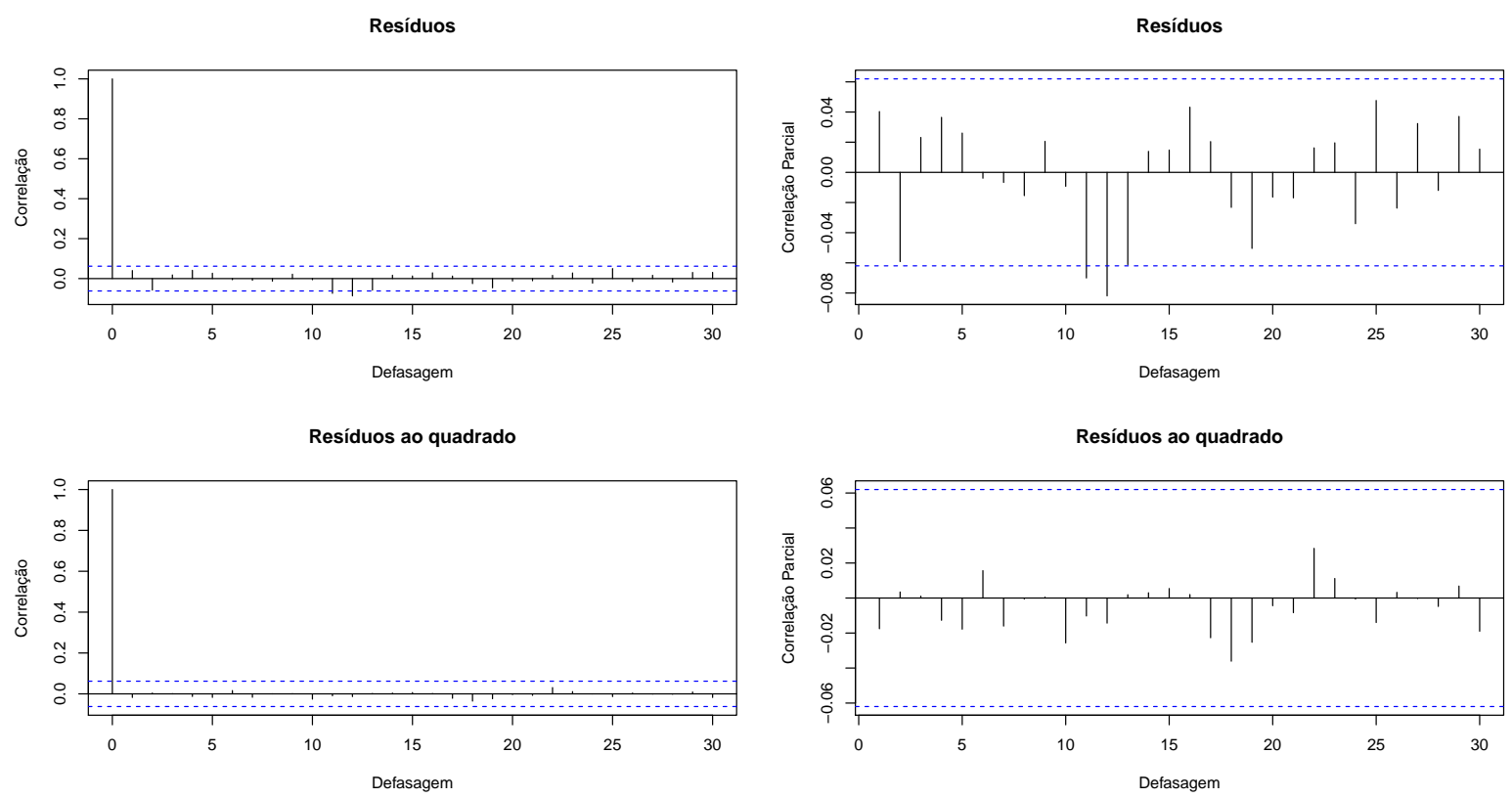

Figura 2.8: Autocorrelação, autocorrelação parcial dos resíduos e dos resíduos ao quadrado para o modelo $\operatorname{AR}(1)$.

Em seguida, estimamos alguns modelos da classe TSARMA, para a série gerada. Na Tabela 2.9, encontramos os valores para os critérios AIC, MSE (erro quadrático médio) e TE (erro total) dos modelos TSARMA usados e do modelo AR(1). Notamos que os valores para esses critérios estão próximos, principalmente, quando comparamos os valores obtidos para os modelos da classe TSARMA. No entanto, quando fazemos uma análise das estimativas das autocorrelações e autocorrelações parciais dos resíduos dos respectivos modelos, Figura 2.9, o modelo que apresenta melhor ajuste é o TSARMA usando a distribuição t-Student com 5 graus de liberdade, (2.22), como esperado, pois foi esse o modelo usado para gerar as observações modeladas:

$$
\begin{array}{cccc}
\hat{y}_{t}^{(0,91)}= & 135,06 & - & 0,005 x_{t} \\
& {[0,2121]} & & \\
& & & \\
0,86\left(y_{t-1}-135,0035\right] & 0,004\left(y_{t-1}-\hat{\eta}_{t-1}\right), & \\
{[0,0146]} & & {[0,0254]} &
\end{array}
$$

em que $\hat{\phi}=33,14$ é o parâmetro de escala estimado e o valor entre parênteses como expoente de $\hat{y}_{t}$ é $\hat{\lambda}$ referente a transformação de Box-Cox. 


\begin{tabular}{rccc}
\hline Modelos & AIC & MSE & TE \\
\hline AR(1) & 7893,74 & 155,78 & $-25,59$ \\
TSARMA-t(5) & 7732,98 & 158,19 & $-1,75$ \\
TSARMA-t(10) & 7757,45 & 159,16 & $-2,01$ \\
TSARMA-t(15) & 7778,57 & 159,21 & $-2,02$ \\
TSARMA-t(20) & 7794,08 & 158,74 & $-1,90$ \\
TSARMA-Normal & 7890,28 & 155,54 & $-0,53$ \\
TSARMA-Logística II & 7674,18 & 158,80 & $-1,87$
\end{tabular}

Tabela 2.9: Valores para algumas medidas de comparação entre modelos.
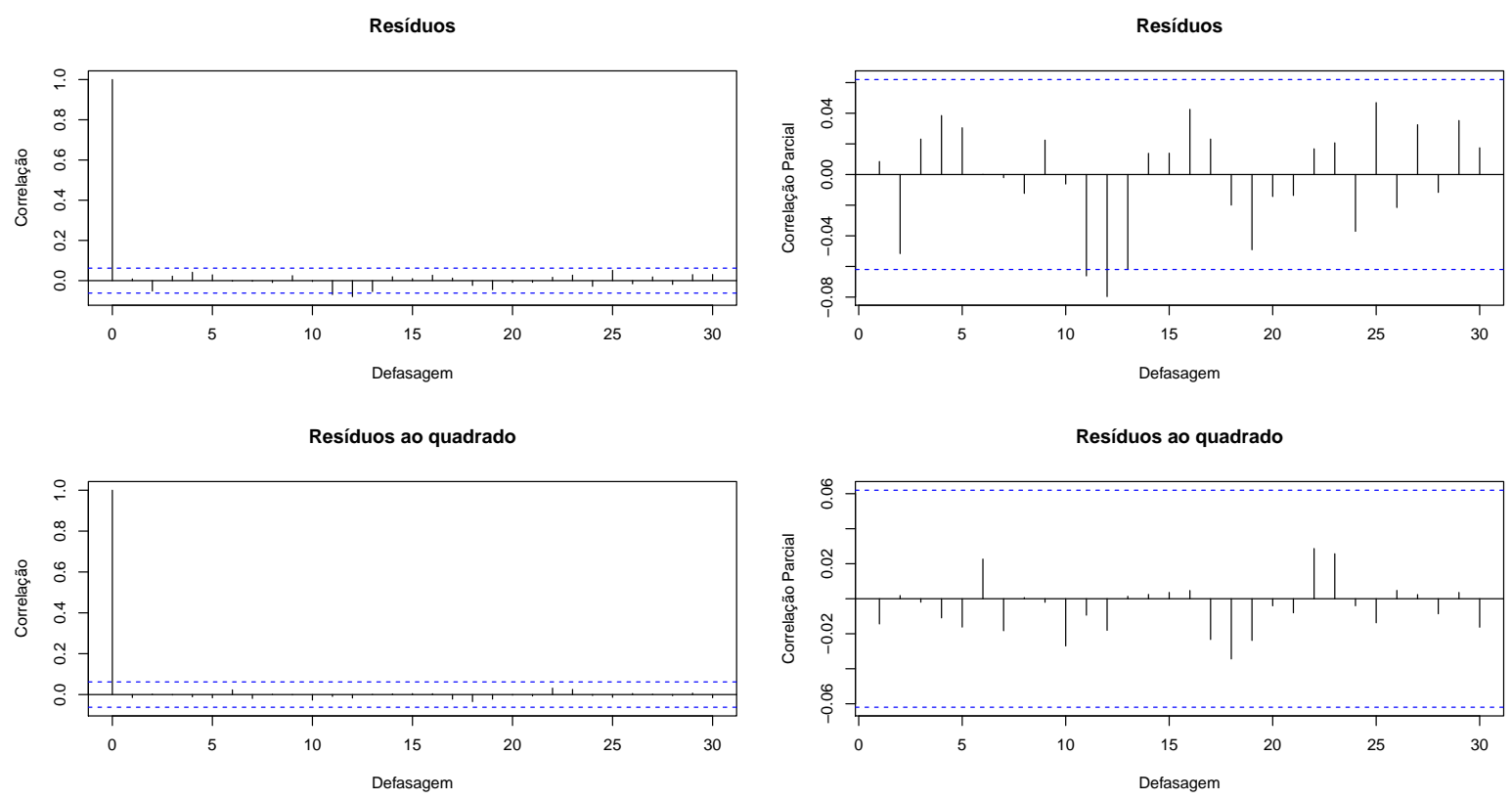

Figura 2.9: Autocorrelação, autocorrelação parcial dos resíduos e dos resíduos ao quadrado para o modelo TSARMA-t(5).

\subsection{Aplicação}

Nesta seção, apresentaremos um exemplo prático com o qual pretendemos avaliar a capacidade preditiva do modelo TSARMA. O conjunto de dados utilizados é o número anual de linces capturados na região do Rio McKenzie, a noroeste do Canadá entre os anos 1821 e 1934.

A inspeção direta do gráfico na Figura 2.10 sugere que os dados têm sazonalidade e não apresentam tendência. De fato, o periodograma desta série, Figura 2.11, revela que o período 
associado ao valor máximo do espectro é 10 anos. Ainda, observando a Figura 2.11, temos indícios, a partir dos gráficos de correlação e correlação parcial, que um modelo AR pode explicar bem o comportamento dessa série, e com o boxplot suspeitamos que a distribuição dos dados é assimétrica.

Inicialmente, estimamos um modelo $\mathrm{AR}(8)$ para a série número anual de linces capturados, um modelo adequado estimado fica como na equação (2.23), de acordo com a análise dos resíduos na Figura 2.12 e com o critério AIC:

$$
\begin{aligned}
& \hat{Y}_{t}=1580,48+1,02 Y_{t-1}-0,49 Y_{t-2}-0,23 Y_{t-7}+0,35 Y_{t-8} \\
& {\left[\begin{array}{lllll}
207,49] & {[0,07]} & {[0,07]} & {[0,07]} & {[0,07]}
\end{array}\right.}
\end{aligned}
$$

em que $\hat{\sigma}_{w}^{2}=635706$ é a variância estimada dos resíduos e os valores entre colchetes são os respectivos desvios padrões das estimativas. A partir, da Figura 2.13 podemos observar o comportamento das estimativas da série na escala original, em torno dos valores reais.

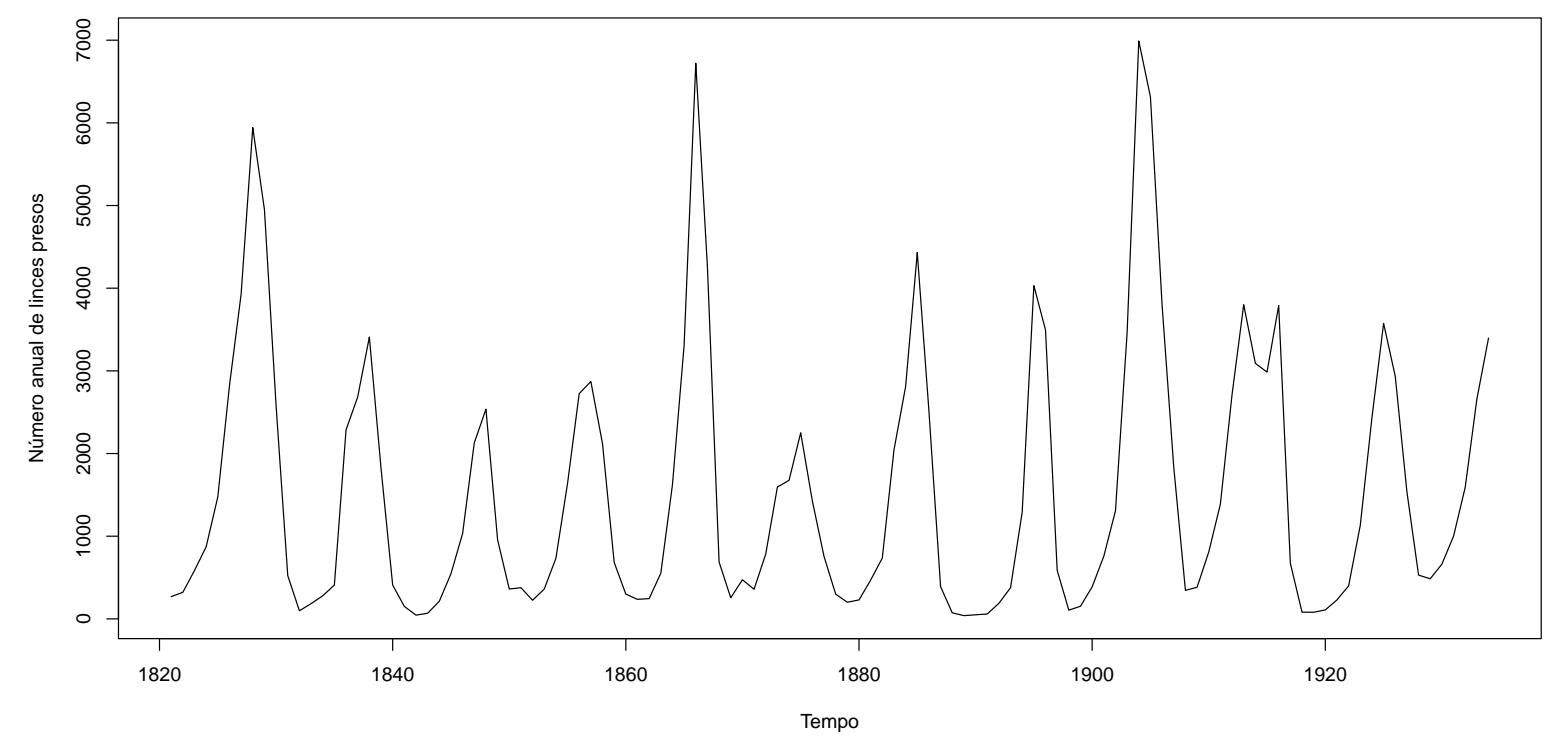

Figura 2.10: Série do número anual de linces presos na região do Rio McKenzie, a noroeste do Canadá.

Em seguida, estimamos alguns modelos da classe TSARMA, para a mesma série. A transformação de Box-Cox foi usada para a variável resposta e consideramos esses dados transformados com distribuição normal, t-Student, logística I e logística II condicional ao passado do processo e para o componente sistemático usamos a função identidade como função de ligação. Dentre os modelos com distribuição normal, o modelo estimado (2.24) foi o que melhor se ajustou aos 

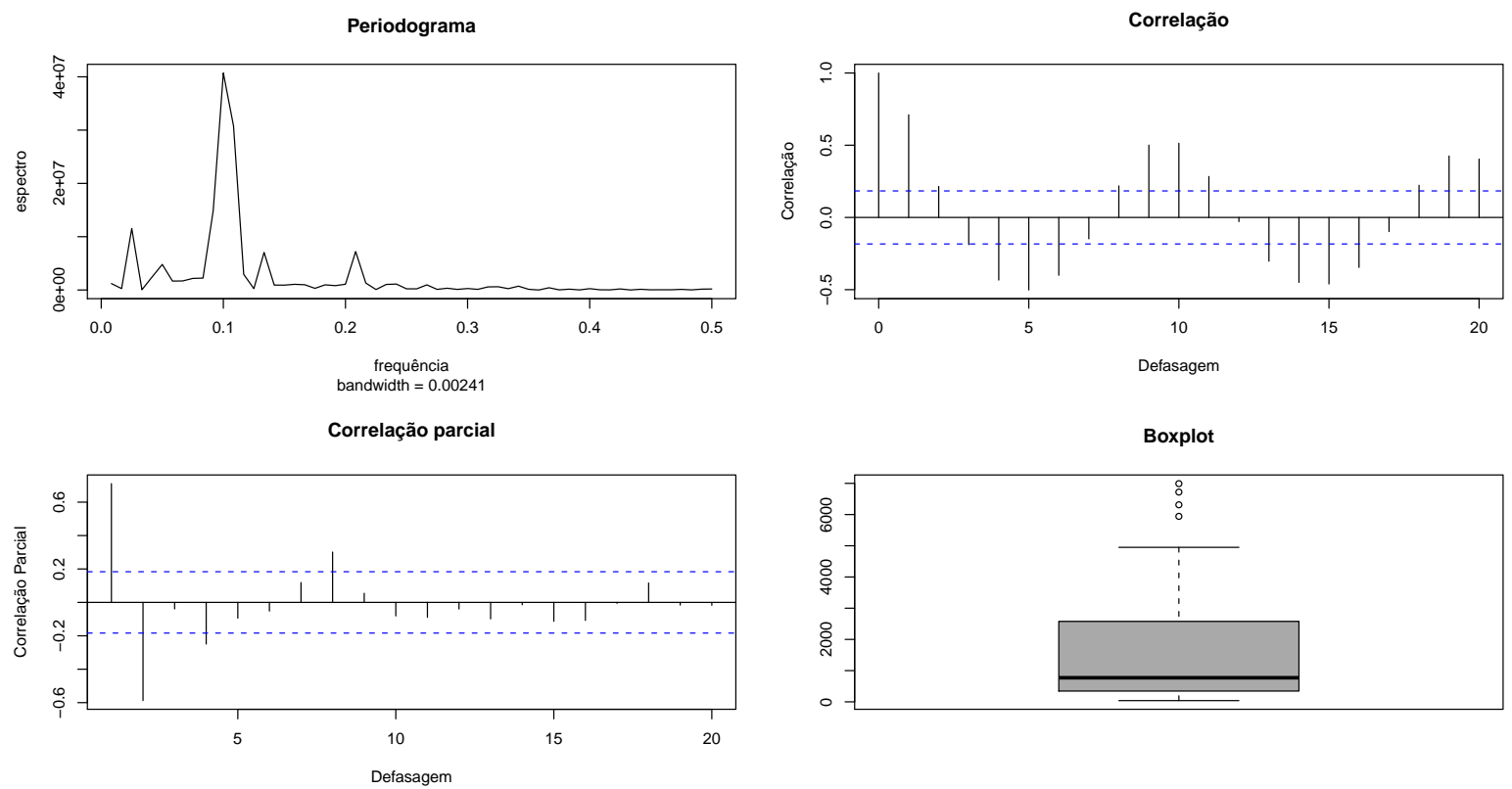

Figura 2.11: Periodograma, autocorrelação, autocorrelação parcial e boxplot da série do número anual de linces presos na região do Rio McKenzie, a noroeste do Canadá.
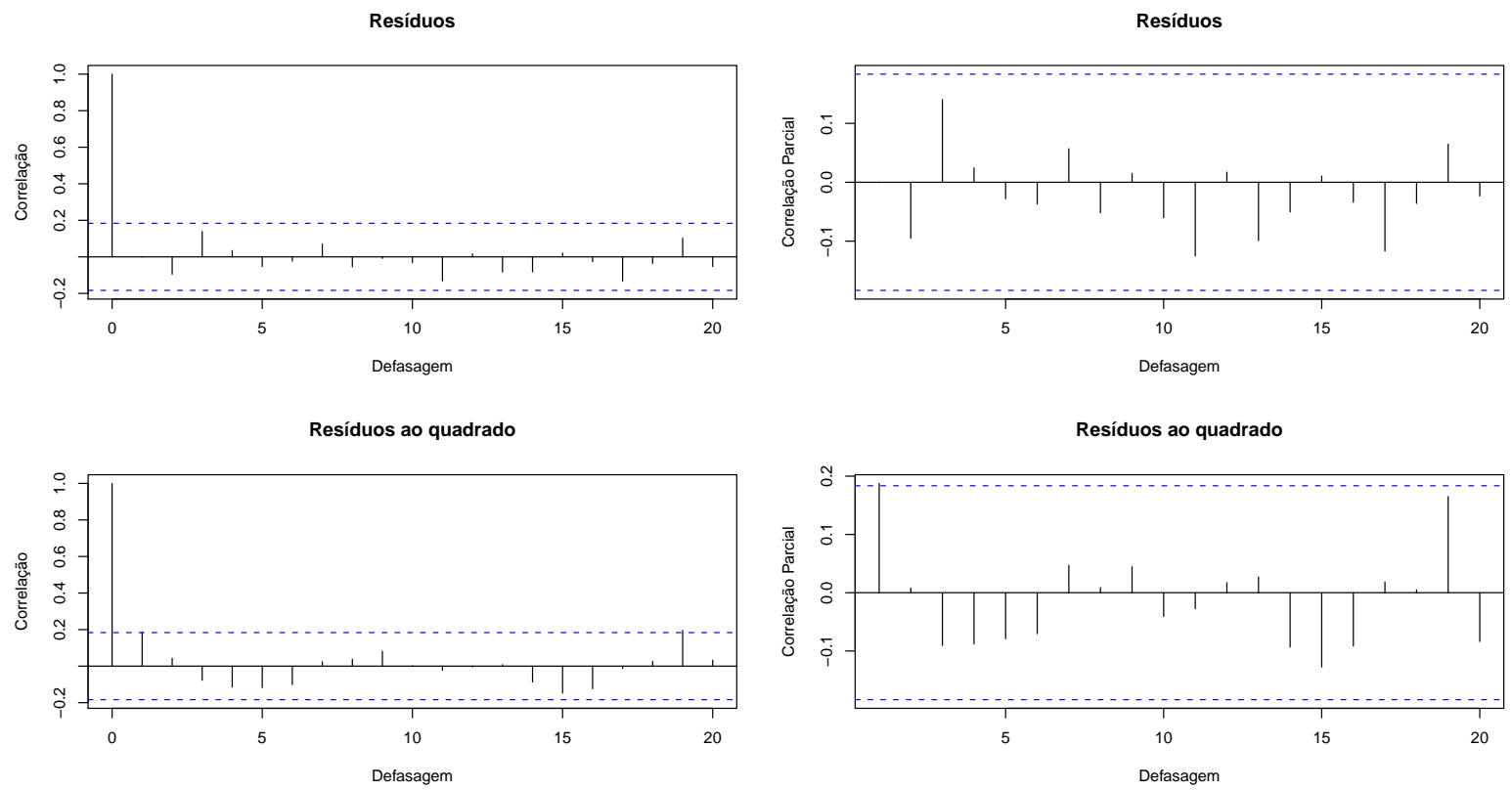

Figura 2.12: Autocorrelação, autocorrelação pacial dos resíduos e dos resíduos ao quadrado para o modelo $\operatorname{AR}(8)$.

dados de acordo com o critério AIC e a análise residual:

$$
\begin{aligned}
& \hat{Y}_{t}^{(0,10)}=3,38+1,19 Y_{t-1}-0,49 Y_{t-2}-0,16 Y_{t-4}+0,11 Y_{t-7}, \\
& {[0,97] \quad[0,09] \quad[0,10] \quad[0,06] \quad[0,05]}
\end{aligned}
$$




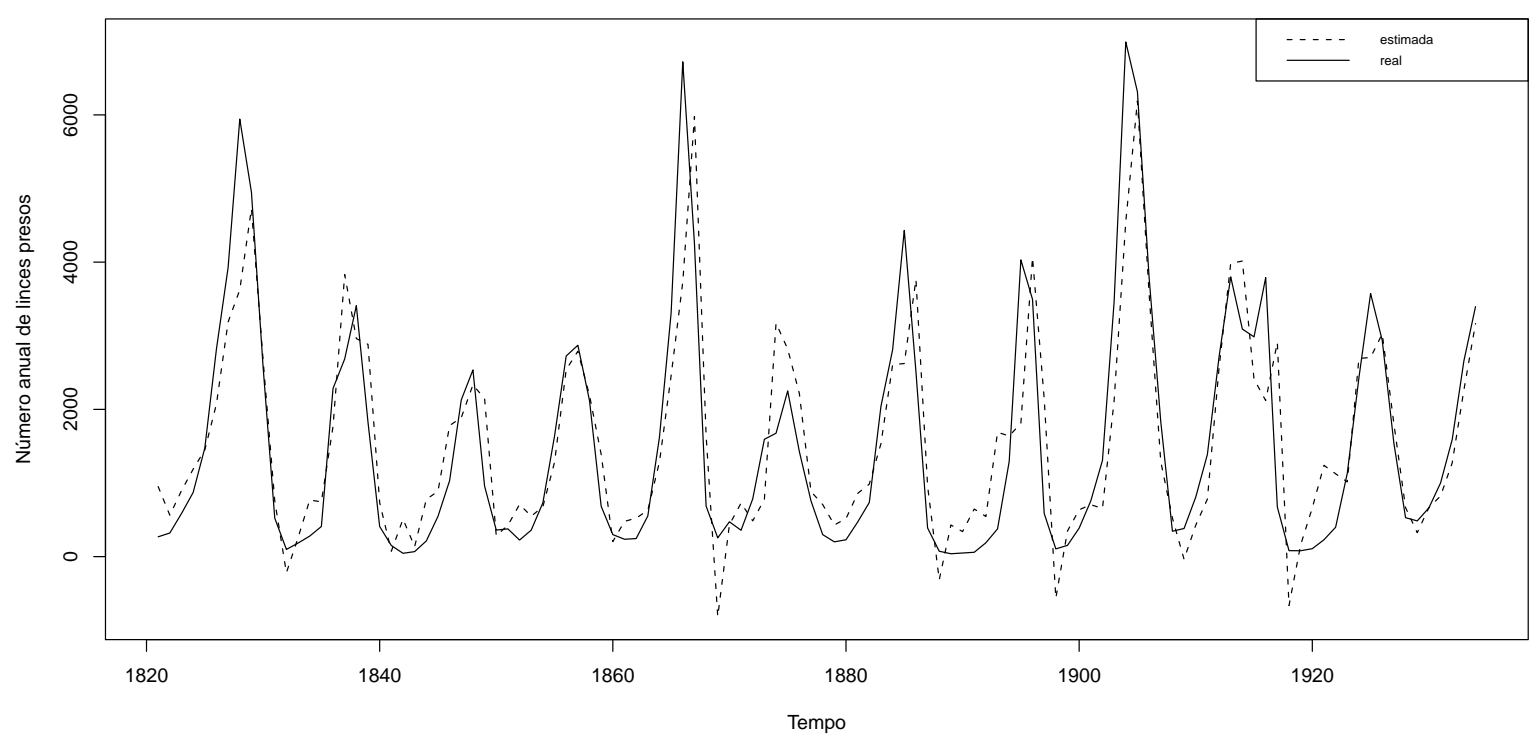

Figura 2.13: Série do número anual de linces presos e estimativas da série na escala original, a partir do modelo $\operatorname{AR}(8)$ ajustado.

em que $\hat{\phi}=0,97$ é o parâmetro de escala estimado, o valor entre parênteses como expoente de $\hat{Y}_{t}$ é $\hat{\lambda}$ referente a transformação de Box-Cox e os valores entre colchetes são os respectivos desvios padrões das estimativas. Na Figura 2.14, podemos observar o comportamento da função de autocorrelação e autocorrelação parcial dos resíduos e dos resíduos ao quadrado, respectivamente. Podemos observar que o gráfico da função de autocorrelação parcial para os resíduos apresenta correlações significativas nas defasagens de ordem 5 e 8 .

Para os modelos TSARMA usando a distribuição t-Student para a variável resposta transformada por Box-Cox, estimamos os modelos com 3, 5, 10, 15, 20, 25 e 30 graus de liberdade. O modelo TSARMA usando distribuição t-Student com 10 graus de liberdade (2.25) foi o que melhor se ajustou aos dados de acordo com a análise residual. Os modelos usando a distribuição t-Student com 15, 20, 25 e 30 graus de liberdade resultaram em resíduos autocorrelacionados, apesar de o valor do critério AIC melhorar a medida que aumentamos os números de graus de liberdade, como vemos na Tabela 2.10 que apresenta os valores para o AIC, MSE (erro quadrático médio) e TE (erro total) dos modelos TSARMA usando t-Student. A partir da Figura 2.16, podemos observar o comportamento das estimativas da série na escala original, em torno dos valores reais. Vale salientar que as estimativas da série na escala original foram obtidas usando a equação (2.12). 

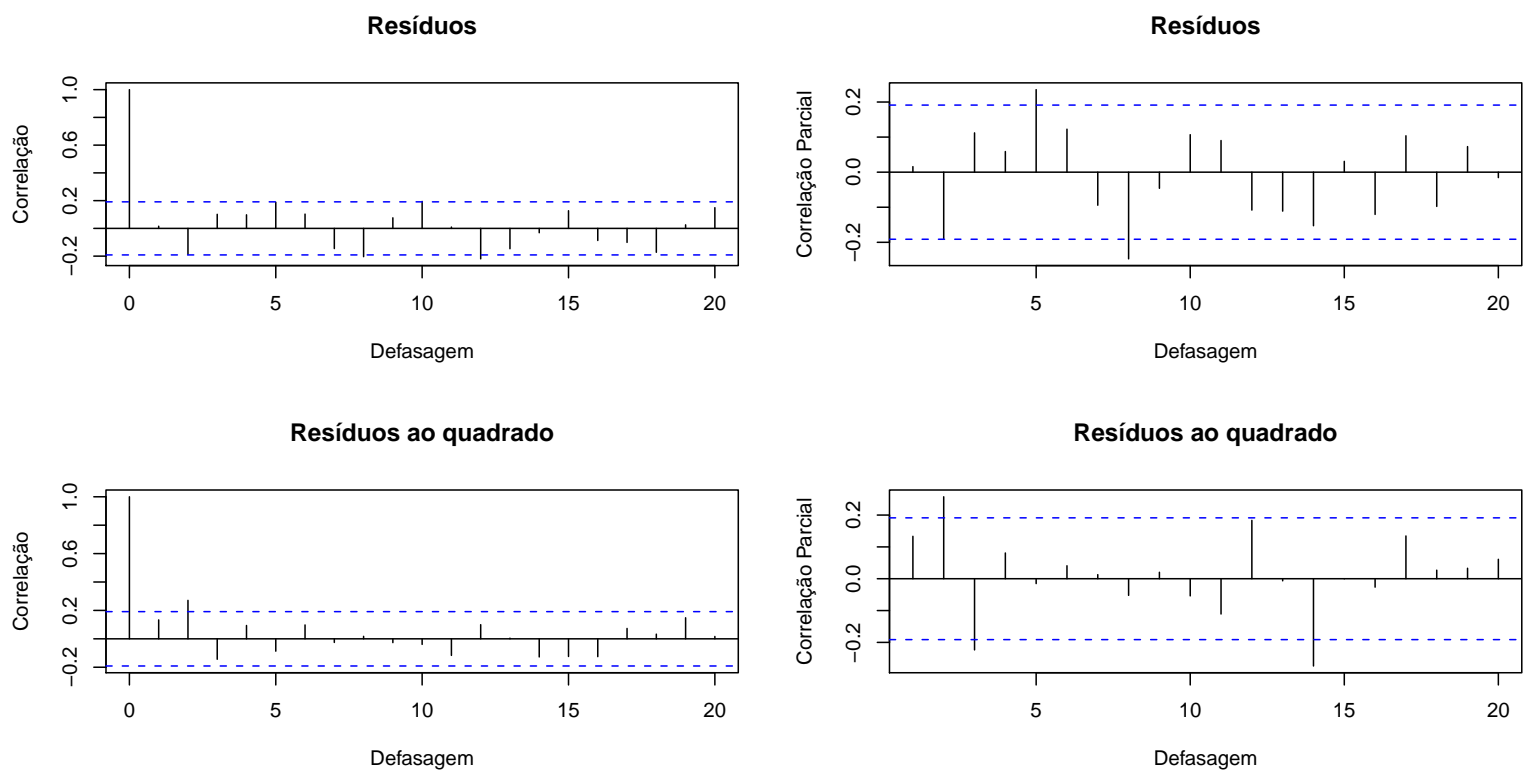

Figura 2.14: Autocorrelação, autocorrelação pacial dos resíduos e dos resíduos ao quadrado para o modelo TSARMA com distribuição normal ajustado.

\begin{tabular}{cccc}
\hline Modelos & AIC & MSE & TE \\
\hline TSARMA-t(3) & 722,532 & 571,477 & 43,92 \\
TSARMA-t(5) & 673,39 & 567,50 & 44,89 \\
TSARMA-t(10) & 626,23 & 567,48 & 46,78 \\
TSARMA-t(15) & 610,80 & 568,13 & 47,88 \\
TSARMA-t(20) & 610,70 & 566,62 & 47,49 \\
TSARMA-t(25) & 610,66 & 565,71 & 47,27 \\
TSARMA-t(30) & 595,56 & 569,26 & 49,53 \\
\hline
\end{tabular}

Tabela 2.10: Valores para algumas medidas de comparação entre modelos.

$$
\begin{aligned}
& \hat{Y}_{t}^{(0,32)}=13,28+1,18 Y_{t-1}-0,56 Y_{t-2}-0,15 Y_{t-4}, \\
& {[1,78] \quad[0,08] \quad[0,09] \quad[0,06]}
\end{aligned}
$$

em que $\hat{\phi}=16,78$ é o parâmetro de escala estimado, o valor entre parênteses como expoente de $\hat{Y}_{t}$ é $\hat{\lambda}$ e os valores entre colchetes são os respectivos desvios padrões das estimativas.

A partir desses resultados, comparamos os dois modelos através de algumas medidas, em particular o erro quadrático médio, MSE, o erro total, TE. Observamos da Tabela 2.11, que 

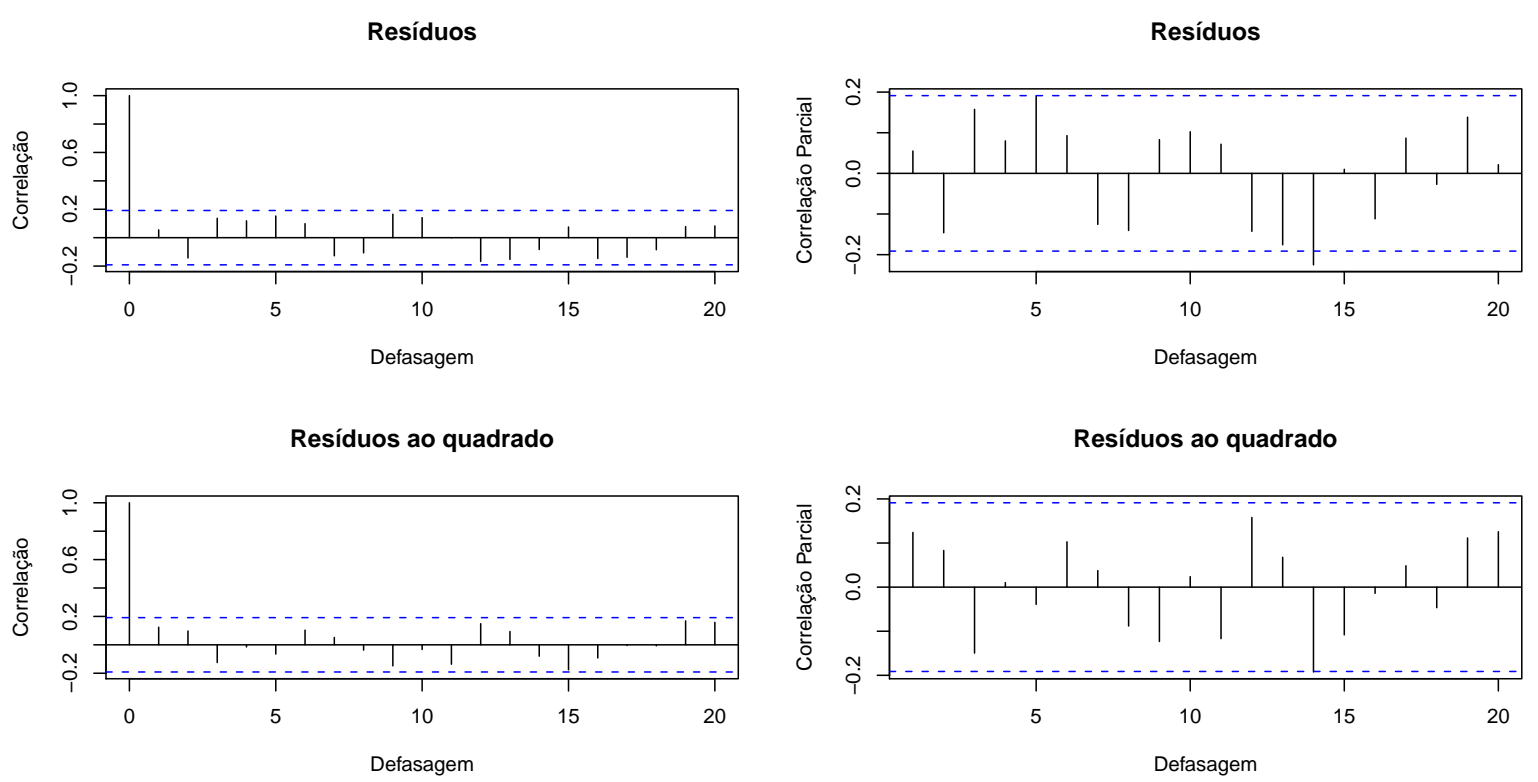

Figura 2.15: Autocorrelação, autocorrelação pacial dos resíduos e dos resíduos ao quadrado para o modelo TSARMA com distribuição t-Student(10) ajustado.

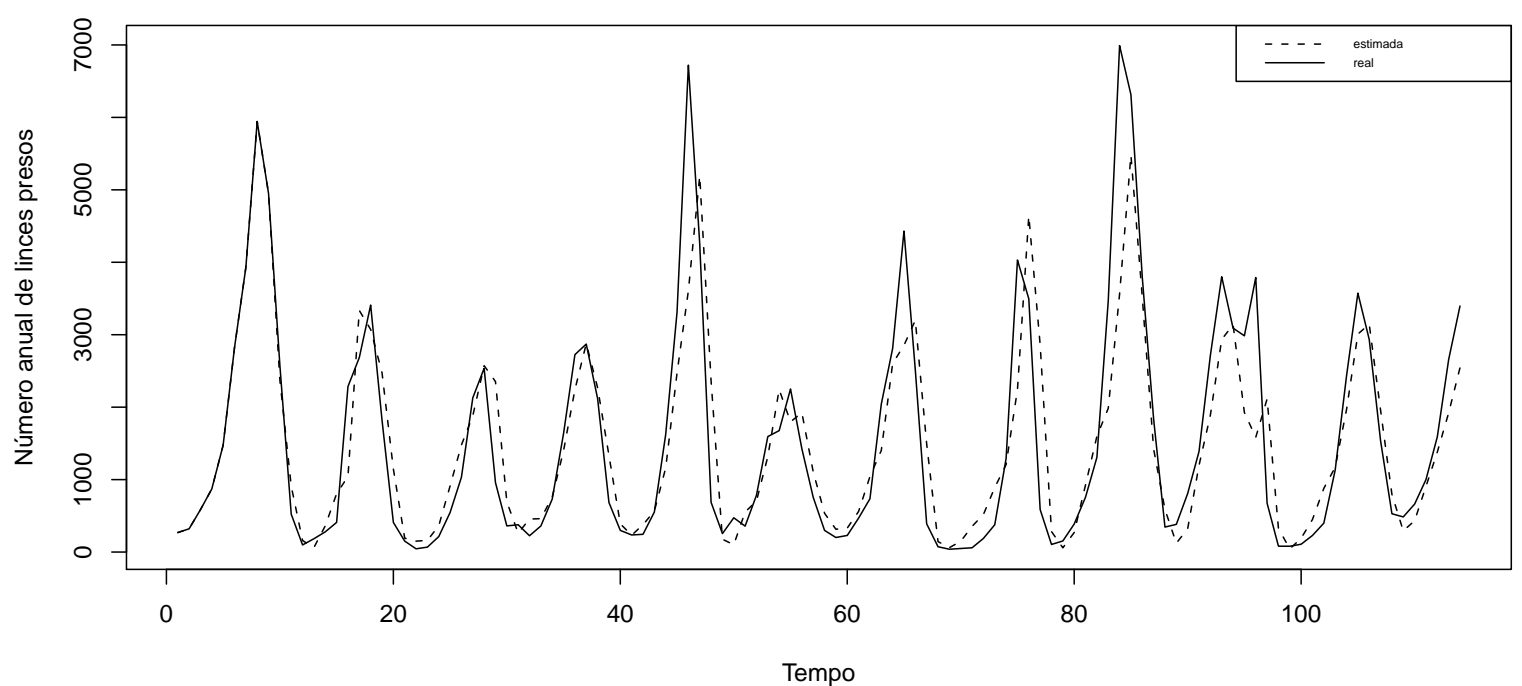

Figura 2.16: Série do número anual de linces presos e estimativas da série na escala original, a partir do modelo TSARMA com distribuição t-Student(10) ajustado.

para todas as medidas usadas, o modelo proposto, TSARMA, supera a modelagem usual de Box e Jenkins. 


\begin{tabular}{ccc}
\hline Medidas de comparação & AR(8) & TSARMA-t(10) \\
\hline AIC & 1861,74 & 626,23 \\
MSE & 635706 & 567877 \\
TE & $-1610,40$ & 49,02
\end{tabular}

Tabela 2.11: Valores para algumas medidas de comparação entre modelos. 


\section{Capítulo 3}

\section{Modelo de Regressão Simétrico Transformado}

\section{Heteroscedástico}

\subsection{Introdução}

Séries temporais econômicas e financeiras apresentam grupos de volatilidade, significando que a variância da série depende do tempo e da informação passada, de modo que temos que definir uma variância condicional, que não sendo constante, não coincide com a variância global (incondicional) da série observada.

Modelos usados para descrever tais séries são os da família ARCH ("autoregressive conditional heteroscedasticity") ou modelos de volatilidade estocástica. Nosso objetivo nesse capítulo é introduzir a classe de modelos simétrico heteroscedástico transformado, como uma alternativa para modelagem de séries temporais econométricas, aliado ao fato de que a transformação potência, também, encontra aplicação prática na determinação empírica de relações funcionais em econometria.

\subsection{Modelo Simétrico Heteroscedástico}

Suponha que a distribuição condicional da variável resposta transformada $\left\{Y_{t}^{(\lambda)}, t=1, \ldots, T\right\}$, dado o passado do processo $H_{t}$, segue uma distribuição simétrica contínua com $\mathrm{E}\left(Y_{t}^{(\lambda)} \mid H_{t}\right)=$ $\mu_{t} \in \mathbb{R}$ e $\operatorname{VAR}\left(Y_{t}^{(\lambda)} \mid H_{t}\right)=\kappa \phi_{t}>0$, ou seja, $Y_{t}^{(\lambda)} \mid H_{t} \sim S\left(\mu_{t}, \phi_{t}\right)$. Assim,

$$
\pi\left(y_{t}^{(\lambda)} \mid H_{t}\right)=\frac{1}{\sqrt{\phi_{t}}} f\left\{\phi_{t}^{-1}\left(y_{t}^{(\lambda)}-\mu_{t}\right)^{2}\right\}
$$

Considere o modelo

$$
y_{t}^{(\lambda)}=\mu_{t}+\sqrt{\phi_{t}} \varepsilon_{t}, t=1, \ldots, T
$$


satisfazendo (3.1) e

$$
\mu_{t}=g^{-1}\left(\eta_{t}\right), \quad \phi_{t}=h^{-1}\left(\tau_{t}\right)
$$

em que

$$
\eta_{t}=x_{t} \beta+\sum_{i=1}^{p} \varphi_{i}\left\{g\left(y_{t-i}^{(\lambda)}\right)-x_{t-i} \beta\right\}+\sum_{i=1}^{q} \psi_{i}\left\{g\left(y_{t-i}^{(\lambda)}\right)-\eta_{t-i}\right\}
$$

e

$$
\tau_{t}=\alpha_{0}+\sum_{i=1}^{r} \alpha_{i} c\left(y_{t-i}^{(\lambda)}, \phi_{t-i}\right)+\sum_{i=1}^{l} \theta_{i} d\left(y_{t-i}^{(\lambda)}, \phi_{t-i}\right)+\sum_{i=1}^{s} \gamma_{i} h\left(\phi_{t-i}\right) .
$$

O modelo Autorregressivo e de Médias Móveis Simétrico Heteroscedástico Transformado, TSHARMA, é definido pela família de transformações (1.1), pela distribuição simétrica (3.1), pelos componentes sistemáticos (3.2) e pelas estruturas de regressão para a média e variância (3.3) e (3.4). Casos especiais do modelo TSHARMA serão considerados em uma seção adiante.

Os parâmetros do modelo são $\beta_{m \times 1}, \varphi_{p \times 1}, \psi_{q \times 1}, \alpha_{(r+1) \times 1}, \theta_{l \times 1}$ e $\gamma_{s \times 1}$. Os estimadores desses parâmetros serão obtidos pelo método de máxima verossimilhança condicional às primeiras $k$ observações transformadas, em que $k=\max \{l, p, q, r, s\}$. Tem-se

$$
L(\nu, \lambda)=\prod_{t=k+1}^{T} \pi\left(y_{t}^{(\lambda)} \mid H_{t}\right) \times J\left(\lambda, y_{t}\right)
$$

sendo $J\left(\lambda, y_{t}\right)=\left|\frac{d \Lambda\left(y_{t} ; \lambda\right)}{d y_{t}}\right|$ o Jacobiano da transformação de $y_{t}$ em $y_{t}^{(\lambda)}$ e $\nu=\left(\beta^{\prime}, \varphi^{\prime}, \psi^{\prime}, \alpha^{\prime}, \theta^{\prime}, \gamma^{\prime}\right)^{\prime}$. Assim, o logaritmo da função de verossimilhança condicional é expresso por

$$
l(\nu, \lambda)=-\frac{1}{2} \sum_{t=k+1}^{T} \log \left(\phi_{t}\right)+\sum_{t=k+1}^{T} \log \left[f\left\{\phi_{t}^{-1}\left(y_{t}^{(\lambda)}-\mu_{t}\right)^{2}\right\}\right]+\sum_{t=k+1}^{T} \log \left\{J\left(\lambda, y_{t}\right)\right\} .
$$

Podemos reescrever o modelo geral para a média $(3.3)$, para $t=k+1, \ldots, T$, como

$$
\eta=\left(\begin{array}{lll}
X & A & B
\end{array}\right)\left(\begin{array}{l}
\beta \\
\varphi \\
\psi
\end{array}\right),
$$

em que 


$$
\begin{aligned}
& \eta=\left(\begin{array}{c}
\eta_{k+1} \\
\eta_{k+2} \\
\vdots \\
\eta_{T}
\end{array}\right)_{(T-k) \times 1}, X=\left(\begin{array}{c}
x_{k+1} \\
x_{k+2} \\
\vdots \\
x_{T}
\end{array}\right)_{(T-k) \times m}, \\
& A=\left(\begin{array}{ccc}
g\left(y_{k}^{(\lambda)}\right)-x_{k} \beta & \cdots & g\left(y_{k+1-p}^{(\lambda)}\right)-x_{k+1-p} \beta \\
g\left(y_{k+1}^{(\lambda)}\right)-x_{k+1} \beta & \cdots & g\left(y_{k+2-p}^{(\lambda)}\right)-x_{k+2-p} \beta \\
\vdots & \ddots & \vdots \\
g\left(y_{T-1}^{(\lambda)}\right)-x_{T-1} \beta & \cdots & g\left(y_{T-p}^{(\lambda)}\right)-x_{T-p} \beta
\end{array}\right)_{(T-k) \times p}
\end{aligned}
$$

$$
B=\left(\begin{array}{ccc}
g\left(y_{k}^{(\lambda)}\right)-\eta_{k} & \cdots & g\left(y_{k+1-q}^{(\lambda)}\right)-\eta_{k+1-q} \\
g\left(y_{k+1}^{(\lambda)}\right)-\eta_{k+1} & \cdots & g\left(y_{k+2-q}^{(\lambda)}\right)-\eta_{k+2-q} \\
\vdots & \ddots & \vdots \\
g\left(y_{T-1}^{(\lambda)}\right)-\eta_{T-1} & \cdots & g\left(y_{T-q}^{(\lambda)}\right)-\eta_{T-q}
\end{array}\right)_{(T-k) \times q}
$$

Então, o modelo geral para a variância (3.4), para $t=k+1, \ldots, T$, pode ser expresso como

$$
\tau=\left(\begin{array}{lll}
C & D & E
\end{array}\right)\left(\begin{array}{l}
\alpha \\
\theta \\
\gamma
\end{array}\right)
$$

em que

$$
\begin{gathered}
\tau=\left(\begin{array}{c}
\tau_{k+1} \\
\tau_{k+2} \\
\vdots \\
\tau_{T}
\end{array}\right)_{(T-k) \times 1}, \quad C=\left(\begin{array}{cccc}
1 & c\left(y_{k}^{(\lambda)}, \phi_{k}\right) & \cdots & c\left(y_{k+1-r}^{(\lambda)}, \phi_{k+1-r}\right) \\
1 & c\left(y_{k+1}^{(\lambda)}, \phi_{k+1}\right) & \cdots & c\left(y_{k+2-r}^{(\lambda)}, \phi_{k+2-r}\right) \\
\vdots & \vdots & \ddots & \vdots \\
1 & c\left(y_{T-1}^{(\lambda)}, \phi_{T-1}\right) & \cdots & c\left(y_{T-r}^{(\lambda)}, \phi_{T-r}\right)
\end{array}\right)_{(T-k) \times(r+1)} \\
D=\left(\begin{array}{ccc}
d\left(y_{k}^{(\lambda)}, \phi_{k}\right) & \cdots & d\left(y_{k+1-l}^{(\lambda)}, \phi_{k+1-l}\right) \\
d\left(y_{k+1}^{(\lambda)}, \phi_{k+1}\right) & \cdots & d\left(y_{k+2-l}^{(\lambda)}, \phi_{k+2-l}\right) \\
\vdots & \ddots & \vdots \\
d\left(y_{T-1}^{(\lambda)}, \phi_{T-1}\right) & \cdots & d\left(y_{T-l}^{(\lambda)}, \phi_{T-l}\right)
\end{array}\right)_{(T-k) \times l}
\end{gathered}
$$




$$
E=\left(\begin{array}{ccc}
h\left(\phi_{k}\right) & \cdots & h\left(\phi_{k+1-s}\right) \\
h\left(\phi_{k+1}\right) & \cdots & h\left(\phi_{k+2-s}\right) \\
\vdots & \ddots & \vdots \\
h\left(\phi_{T-1}\right) & \cdots & h\left(\phi_{T-s}\right)
\end{array}\right)_{(T-k) \times s}
$$

Assim, as estruturas de regressão para a média e para a variância podem ser reescritas como

$$
\eta=M^{(\lambda)} \nu_{1} \quad \text { e } \quad \tau=N^{(\lambda)} \nu_{2}
$$

em que

$$
M^{(\lambda)}=\left(\begin{array}{ccc}
X & A & B
\end{array}\right), N^{(\lambda)}=\left(\begin{array}{ccc}
C & D & E
\end{array}\right), \nu_{1}=\left(\begin{array}{l}
\beta \\
\varphi \\
\psi
\end{array}\right) \quad \text { e } \quad \nu_{2}=\left(\begin{array}{l}
\alpha \\
\theta \\
\gamma
\end{array}\right)
$$

Para maximizar o logaritmo da função de verossimilhança condicional (3.5), assumiremos, inicialmente, que o parâmetro de transformação, $\lambda$, é fixo. Assim, dado o parâmetro de transformação $\lambda$, as estimativas de máxima verossimilhança condicional são $\hat{\nu}^{(\lambda)}=\left(\begin{array}{c}\hat{\nu}_{1}^{(\lambda)} \\ \hat{\nu}_{2}^{(\lambda)}\end{array}\right), \hat{\eta}^{(\lambda)}=$ $\hat{M}^{(\lambda)} \hat{\nu}_{1}^{(\lambda)}, \hat{\mu}^{(\lambda)}=g^{-1}\left(\hat{\eta}^{(\lambda)}\right), \hat{\tau}^{(\lambda)}=\hat{N}^{(\lambda)}{\hat{\nu_{2}}}^{(\lambda)}$ e $\hat{\phi}^{(\lambda)}=h\left(\hat{\tau}^{(\lambda)}\right)$ de $\nu=\left(\begin{array}{c}\nu_{1} \\ \nu_{2}\end{array}\right), \eta, \mu, \tau$ e $\phi$, respectivamente.

As funções escore para $\nu_{1}$ e $\nu_{2}$ têm, respectivamente, as formas

$$
U_{\nu_{1}}(\nu)=M^{(\lambda) T} H D^{(\lambda)}\left(y^{(\lambda)}-\mu\right) \quad \text { e } \quad U_{\nu_{2}}(\nu)=N^{(\lambda) T} Q^{(\lambda)},
$$

em que $\quad H=\operatorname{diag}\left\{\mu_{k+1}^{\prime}, \ldots, \mu_{T}^{\prime}\right\}, \quad \mu_{i}^{\prime}=\frac{d \mu_{i}}{d \eta_{i}}, \quad D^{(\lambda)}=\operatorname{diag}\left\{g_{k+1}, \ldots, g_{T}\right\}, \quad g_{i}=\frac{v_{i}}{\phi_{i}}=\frac{-2 W_{f}\left(u_{i}\right)}{\phi_{i}}=$

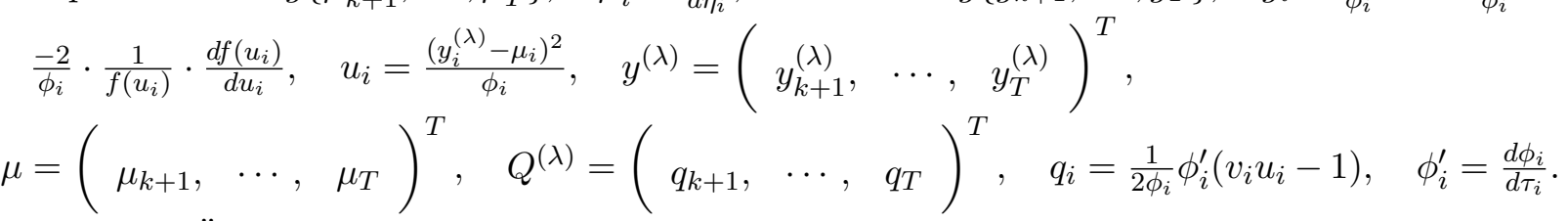

Seja $-\left.\ddot{L}_{\nu \nu}\right|_{\hat{\nu}}$ a matriz de informação observada de Fisher para $\nu$ expressa como

$$
\ddot{L}_{\nu \nu}=\left(\begin{array}{ll}
\ddot{L}_{\nu_{1} \nu_{1}} & \ddot{L}_{\nu_{1} \nu_{2}} \\
\ddot{L}_{\nu_{2} \nu_{1}} & \ddot{L}_{\nu_{2} \nu_{2}}
\end{array}\right)
$$

em que $\quad \ddot{L}_{\nu_{1} \nu_{1}}=M^{(\lambda) T} D(a) M^{(\lambda)}, \quad \ddot{L}_{\nu_{1} \nu_{2}}=2 M^{(\lambda) T} D(b) N^{(\lambda)} \quad$ e $\quad \ddot{L}_{\nu_{2} \nu_{2}}=N^{(\lambda) T} D(c) N^{(\lambda)}$ 
$\operatorname{com} \quad D(a)=\operatorname{diag}\left\{a_{k+1}, \ldots, a_{T}\right\}, \quad D(b)=\operatorname{diag}\left\{b_{k+1}, \ldots, b_{T}\right\}, \quad D(c)=\operatorname{diag}\left\{c_{k+1}, \ldots, c_{T}\right\}$, $a_{i}=\frac{4 u_{i}}{\phi_{i}} W_{f}^{\prime}\left(u_{i}\right) \mu_{i}^{\prime 2}-\frac{v_{i}}{\phi_{i}} \mu_{i}^{\prime 2}+\frac{v_{i}}{\phi_{i}} \mu_{i}^{\prime \prime}\left(y_{i}^{(\lambda)}-\mu_{i}\right), \quad \mu_{i}^{\prime \prime}=\frac{d^{2} \mu_{i}}{d \eta_{i}^{2}}, \quad b_{i}=\frac{\phi_{i}^{\prime}}{\phi_{i}^{2}}\left[W_{f}^{\prime}\left(u_{i}\right) u_{i}+W_{f}\left(u_{i}\right)\right]\left(y_{i}^{(\lambda)}-\right.$ $\left.\mu_{i}\right), \quad W_{f}^{\prime}\left(u_{i}\right)=\frac{d W_{f}\left(u_{i}\right)}{d u_{i}}, \quad c_{i}=\frac{\left(v_{i} u_{i}-1\right)}{2 \phi_{i}} \phi_{i}^{\prime \prime}+\frac{\left(1+2 W_{f}^{\prime}\left(u_{i}\right) u_{i}^{2}-2 v_{i} u_{i}\right)}{2 \phi_{i}^{2}} \phi_{i}^{\prime 2}$.

Podemos mostrar que a matriz de informação esperada de Fisher $K_{\nu \nu}=\mathrm{E}\left(-\ddot{L}_{\nu \nu}\right)$ para $\nu$ é bloco-diagonal, $K_{\nu \nu}=\operatorname{diag}\left\{K_{\nu_{1} \nu_{1}}, K_{\nu_{2} \nu_{2}}\right\}$, ou seja, os vetores de parâmetros $\nu_{1}$ e $\nu_{2}$ são globalmente ortogonais. As matrizes de informação esperada de Fisher $K_{\nu_{1} \nu_{1}}$ e $K_{\nu_{2} \nu_{2}}$ para $\nu_{1}$ e $\nu_{2}$ são expressas, respectivamente, por

$$
K_{\nu_{1} \nu_{1}}=4 d_{g} M^{(\lambda) T} H^{2} \Phi^{-1} M^{(\lambda)} \quad \text { e } \quad K_{\nu_{2} \nu_{2}}=\frac{\left(4 f_{g}-1\right)}{4} N^{(\lambda) T} D_{\phi}^{2} \Phi^{-2} N^{(\lambda)}
$$

em que $\Phi=\operatorname{diag}\left\{\phi_{k+1}, \ldots, \phi_{T}\right\}, \quad D_{\phi}=\operatorname{diag}\left\{\phi_{k+1}^{\prime}, \ldots, \phi_{T}^{\prime}\right\}, \quad d_{g}=\mathrm{E}\left\{W_{f}^{2}\left(U^{2}\right) U^{2}\right\} \quad$ e $f_{g}=$ $\mathrm{E}\left\{W_{f}^{2}\left(U^{2}\right) U^{4}\right\}, \quad$ sendo $U \sim S(0,1)$.

Um processo iterativo para produzir as EMVs de $\nu_{1}$ e $\nu_{2}$ é o método escore de Fisher. As EMVs $\hat{\nu}_{1}^{(\lambda)}$ e $\hat{\nu}_{2}^{(\lambda)}$ podem ser obtidas, resolvendo-se o seguinte sistema de equações:

$$
\begin{aligned}
& M^{(\lambda) T(m)} H^{2(m)} \Phi^{(m)(-1)} M^{(\lambda)(m)} \nu_{1}^{(\lambda)(m+1)}=M^{(\lambda) T(m)} H^{2(m)} \Phi^{(m)(-1)} z_{\nu_{1}}^{(\lambda)(m)} \\
& N^{(\lambda) T(m)} D_{\phi}^{2(m)} \Phi^{(m)(-2)} N^{(\lambda)(m)} \nu_{2}^{(\lambda)(m+1)}=N^{(\lambda) T(m)} D_{\phi}^{2(m)} \Phi^{(m)(-2)} z_{\nu_{2}}^{(\lambda)(m)}
\end{aligned}
$$

em que $z_{\nu_{1}}^{(\lambda)(m)}$ e $z_{\nu_{2}}^{(\lambda)(m)}$ são vetores $(T-k) \times 1$ expressos por

$$
z_{\nu_{1}}^{(\lambda)(m)}=\eta^{(m)}+\left(4 d_{g}\right)^{-1} H^{(m)(-1)} V^{(m)}\left(y^{(\lambda)}-\mu^{(m)}\right)
$$

e

$$
z_{\nu_{2}}^{(\lambda)(m)}=\tau^{(m)}+\frac{2}{\left(4 f_{g}-1\right)} D_{\phi}^{2(m)(-1)} \Phi^{(m)} P^{(\lambda)(m)}
$$

sendo que $V^{(m)}=\operatorname{diag}\left\{v_{k+1}^{(m)}, \ldots, v_{T}^{(m)}\right\}$ e $P^{(\lambda)(m)}=\left(p_{k+1}^{(\lambda)(m)}, \quad \cdots, \quad p_{T}^{(\lambda)(m)}\right)^{T}, p_{i}=v_{i} u_{i}-1$. Expressões para $W_{f}(u)$ e $W_{f}^{\prime}(u)$ e valores para $d_{g}$ e $f_{g}$ para algumas distribuições simétricas são apresentados nas Tabelas 2.1 e 2.2. Maiores detalhes podem ser encontrados em Cysneiros e Paula (2005).

Substituindo a EMV condicional $\hat{\nu}^{(\lambda)}=\left(\begin{array}{c}\hat{\nu}_{1}^{(\lambda)} \\ \hat{\nu}_{2}^{(\lambda)}\end{array}\right)$ em (3.5), obtém-se o logaritmo da função de verossimilhança perfilada para $\lambda$ 


$$
l_{p}(\lambda)=-\frac{1}{2} \sum_{t=k+1}^{T} \log \left(\hat{\phi}_{t}^{(\lambda)}\right)+\sum_{t=k+1}^{T} \log \left[f\left\{\hat{\phi}_{t}^{(\lambda)(-1)}\left(y_{t}^{(\lambda)}-\hat{\mu}_{t}^{(\lambda)}\right)^{2}\right\}\right]+\sum_{t=k+1}^{T} \log \left\{J\left(\lambda, y_{t}\right)\right\} .
$$

Seja $s_{t}^{(\lambda) 2}=f\left\{\left(\hat{\phi}_{t}^{(\lambda)(-1)}\left(y_{t}^{(\lambda)}-\hat{\mu}_{t}^{(\lambda)}\right)^{2}\right)\right\}$ e defina as médias geométricas

$$
\tilde{s}^{(\lambda) 2}=\left(\prod_{t=k+1}^{T} s_{t}^{(\lambda) 2}\right)^{\frac{1}{T-k}} \text { e } \tilde{J}^{(\lambda)}=\left(\prod_{t=k+1}^{T} \frac{J\left(\lambda, y_{t}\right)}{\hat{\phi}_{t}^{(\lambda)\left(\frac{1}{2}\right)}}\right)^{\frac{1}{T-k}} .
$$

Então, o logaritmo da função de verossimilhança perfilada fica reduzida a

$$
l_{p}(\lambda)=(T-k) \log \left(\tilde{s}^{(\lambda) 2} \tilde{J}^{(\lambda)}\right)
$$

O logaritmo da função de verossimilhança perfilada em (3.7) é maximizada numericamente, por meio do gráfico de $l_{p}(\lambda)$ versus $\lambda$, para uma grade de valores para $\lambda$. Para maximizar (3.7), necessitamos encontrar um valor de $\lambda$ que minimize a razão

$$
\hat{\lambda}=\arg \min _{\lambda}\left(\tilde{s}^{(\lambda) 2} \tilde{J}^{(\lambda)}\right)^{-1}
$$

Podemos substituir a EMV nas equações (3.6) e encontrar as estimativas irrestritas de $\hat{\nu}=$ $\hat{\nu}^{(\hat{\lambda})}$ e, assim, o logaritmo da função de verossimilhança maximizado $\hat{l}=l_{p}(\hat{\lambda})$ segue de $(3.7)$ para o modelo considerado.

\subsection{Modelos TSHARMA Especiais}

Os modelos autorregressivos com heteroscedasticidade condicional, ARCH, foram introduzidos por Engle (1982), com o objetivo de estimar a variância da inflação. Uma generalização dos modelos ARCH foi sugerida por Bollerslev (1986, 1987, 1988), o chamado modelo GARCH, Generalized ARCH. A ideia básica, nessa classe de modelos é que a série não é autocorrelacionada, mas a variância condicional depende do passado da série. Quando a série é autocorrelacionada usam-se modelos ARMA-GARCH, pois assim a parte ARMA do modelo trata a autocorrelação serial, enquanto a parte GARCH trata da variância condicional da série. Na prática, usualmente, supõe-se a distribuição normal ou a t-Student. Os modelos ARCH e GARCH modelam a variância condicional de forma simétrica, pois a mesma é uma função quadrática da série, porém muitas vezes a variância condicional reage de forma assimétrica aos valores da série, tendendo a ser maior para valor negativo. Assim, foram criados modelos com a finalidade de tratar a 
série assimetricamente, e os modelos mais usados, na prática, com esse objetivo, são os modelos EGARCH, TARCH e PGARCH. Nelson (1991) introduziu os modelos EGARCH, exponencial GARCH e Ding et al. (1993) propôs o modelo PGARCH $(1,1)$, power GARCH. Para mais detalhes do modelo TARCH, vide Zakoian (1994) e Glosten, Jagannathan e Runkle (1993).

O modelo TSHARMA tem uma forma interessante quando tratamos dos modelos da classe GARCH, ou seja, o modelo TSHARMA pode ser usado como uma extensão dos modelos GARCH para as distribuições simétricas contínuas.

Um modelo $\operatorname{GARCH}(r, s)$ é definido por

$$
\begin{gathered}
X_{t}=\sqrt{h_{t}} \varepsilon_{t} \\
h_{t}=\alpha_{0}+\sum_{i=1}^{r} \alpha_{i} X_{t-i}^{2}+\sum_{j=1}^{s} \beta_{j} h_{t-j},
\end{gathered}
$$

em que $\varepsilon_{t}$ i.i.d $(0,1), \alpha_{0}>0, \alpha_{i} \geq 0, \beta_{j} \geq 0, \sum_{i=1}^{q}\left(\alpha_{i}+\beta_{i}\right)<1, q=\max \{r, s\}$.

Os coeficientes positivos dão uma condição suficiente, mas não necessária, para que $h_{t}>0$. Para condições gerais, vide Nelson e Cao (1992).

Quando $\beta_{j}=0, \forall j>0$ e $\alpha_{0}>0, \alpha_{i} \geq 0, \forall i>0$, temos um modelo $\operatorname{ARCH}(r)$.

Assim, considerando no componente sistemático $(3.2), h\left(\tau_{t}\right)=\tau_{t}$ e no modelo geral para a variância (3.4), $c\left(y_{t-i}^{(\lambda)}, \phi_{t-i}\right)=y_{t-i}^{(\lambda) 2}$ e $\theta_{i}=0, \forall i>0$, temos uma extensão do modelo GARCH e quando $\theta_{i}=\gamma_{i}=0, \forall i>0$, em (3.4), temos uma extensão do modelo ARCH.

Considerando $h\left(\tau_{t}\right)=\exp \left(\tau_{t}\right)$ em $(3.2)$, em $(3.4)$

$$
c\left(y_{t-i}^{(\lambda)}, \phi_{t-i}\right)=\left|\frac{y_{t-i}^{(\lambda)}}{\sqrt{\phi_{t-i}}}\right| \quad \text { e } \quad d\left(y_{t-i}^{(\lambda)}, \phi_{t-i}\right)=\frac{y_{t-i}^{(\lambda)}}{\sqrt{\phi_{t-i}}},
$$

a equação (3.4) pode ser expressa como

$$
\tau_{t}=\ln \left(\phi_{t}\right)=w+\sum_{i=1}^{r} \alpha_{i}\left|\frac{y_{t-i}^{(\lambda)}}{\sqrt{\phi_{t-i}}}\right|+\sum_{i=1}^{l} \theta_{i} \frac{y_{t-i}^{(\lambda)}}{\sqrt{\phi_{t-i}}}+\sum_{i=1}^{s} \gamma_{i} \tau_{t-i}
$$

que pode ser vista como uma extensão do modelo EGARCH estendido, usado pelo EViews, sendo que os coeficientes de alavancagem são os $\theta_{i}$. 
Considerando em (3.4),

$$
c\left(y_{t-i}^{(\lambda)}, \phi_{t-i}\right)=y_{t-i}^{(\lambda) 2} \quad \text { e } \quad d\left(y_{t-i}^{(\lambda)}, \phi_{t-i}\right)=\left\{\begin{array}{ccc}
y_{t-i}^{(\lambda) 2} & \text { se } & y_{t-i}^{(\lambda)}<0 \\
0 & \text { se } & y_{t-i}^{(\lambda)} \geq 0
\end{array}\right.
$$

com $h\left(\tau_{t}\right)=\tau_{t}$, temos uma extensão do modelo TARCH, no qual há impacto de informação assimétrica quando $\theta_{i} \neq 0$, esperamos que $\theta_{i}>0$ para que observações negativas tenham um impacto maior na variância condicional.

Além disso, temos que o modelo TSHARMA é uma extensão natural da classe de modelos GARMA desenvolvida por Benjamin et al. (2003) e da classe de modelos TGARMA proposta por Cordeiro e Andrade (2009).

\subsection{Simulações}

O principal objetivo desta seção é avaliar o comportamento das estimativas dos modelos TSHARMA usando algumas distribuições simétricas. Para isso, realizamos algumas simulações, no programa computacional R. Assim como na Seção 2.7, avaliaremos as estimativas de acordo com o viés e o erro quadrático médio, EQM.

O modelo (3.11) foi usado para gerar um conjunto de dados heteroscedasticos, cuja distribuição condicional é normal, com os componentes sistemáticos para a média e variância com a função de ligação sendo a função identidade, expressão (3.12).

$$
y_{t}^{(1)}=\mu_{t}+\sqrt{\phi_{t}} \varepsilon_{t}, \quad \varepsilon_{t} \sim N(0,1)
$$

satisfazendo

$$
\begin{aligned}
& \mu_{t}=\eta_{t}=20+0,8 \times\left(y_{t-1}-20\right), \\
& \phi_{t}=\tau_{t}=0,2+0,6 \times \phi_{t-1} \varepsilon_{t-1}^{2} .
\end{aligned}
$$

Na Tabela 3.1, podemos observar o desempenho das estimativas com os seus respectivos vieses e erros quadrático médio, EQM, para o modelo transformado ( 3.11 ), em que o número de réplicas é 200 e o tamanho das amostras é $T=500$. Observamos que para os parâmetros $\varphi=0,8$ e $\alpha_{1}=0,6$ os seus respectivos vieses e EQM são pequenos, ao passo que, para os demais parâmetros houve grandes diferenças, pricipalmente, no que diz respeito aos interceptos para as estruturas de regressão para a média e para a variância. Quando o parâmetro de transformação é estimado com grandes desvios do valor verdadeiro, causa uma mudança na grandeza dos dados 
e, necessariamente, as estimativas que dependem da escala dos dados sofrem grandes desvios dos seus valores verdadeiros, o que pode ser melhor notado nas Figuras 3.1 e 3.2. A Figura 3.1 apresenta os histogramas e os QQ-plots construídos com as estimativas dos parâmetros $\lambda$, $\beta_{0}$, e $\varphi$ a partir das 200 réplicas usando o modelo (3.11) com $T=500$. Podemos ver que as estimativas para o parâmetro $\varphi$ se comportam bem próximo de uma distribuição normal, enquanto que as demais tem um comportamento de uma distribuição com caudas mais pesadas que a normal. $\mathrm{O}$ mesmo podemos observar na Figura 3.2, que mostra o comportamento das estimativas para $\alpha_{0}$ e $\alpha_{1}$. Vemos que as estimativas para o parâmetro $\alpha_{1}$ tem um comportamento próximo ao da distribuição normal, ao passo que as estimativas para o parâmetro $\alpha_{0}$ tem um comportamento assimétrico.

\begin{tabular}{crrrrr}
\hline$T$ & \multicolumn{1}{c}{$\hat{\lambda}$} & \multicolumn{1}{c}{$\hat{\beta}_{0}$} & $\hat{\varphi}$ & \multicolumn{1}{c}{$\hat{\alpha_{0}}$} & \multicolumn{1}{c}{$\hat{\alpha_{1}}$} \\
\hline & & & & & \\
500 & 1,059 & 32,788 & 0,795 & 4,097 & 0,592 \\
Viés & $-0,059$ & $-12,788$ & 0,005 & $-3,897$ & 0,008 \\
EQM & 0,117 & 1597,891 & 0,001 & 233,305 & 0,009
\end{tabular}

Tabela 3.1: Valores para os EMV dos respectivos parâmetros $\lambda=1, \beta_{0}=20, \varphi=0,8, \alpha_{0}=0,2$ e $\alpha_{1}=$ 0,6 para distribuição normal
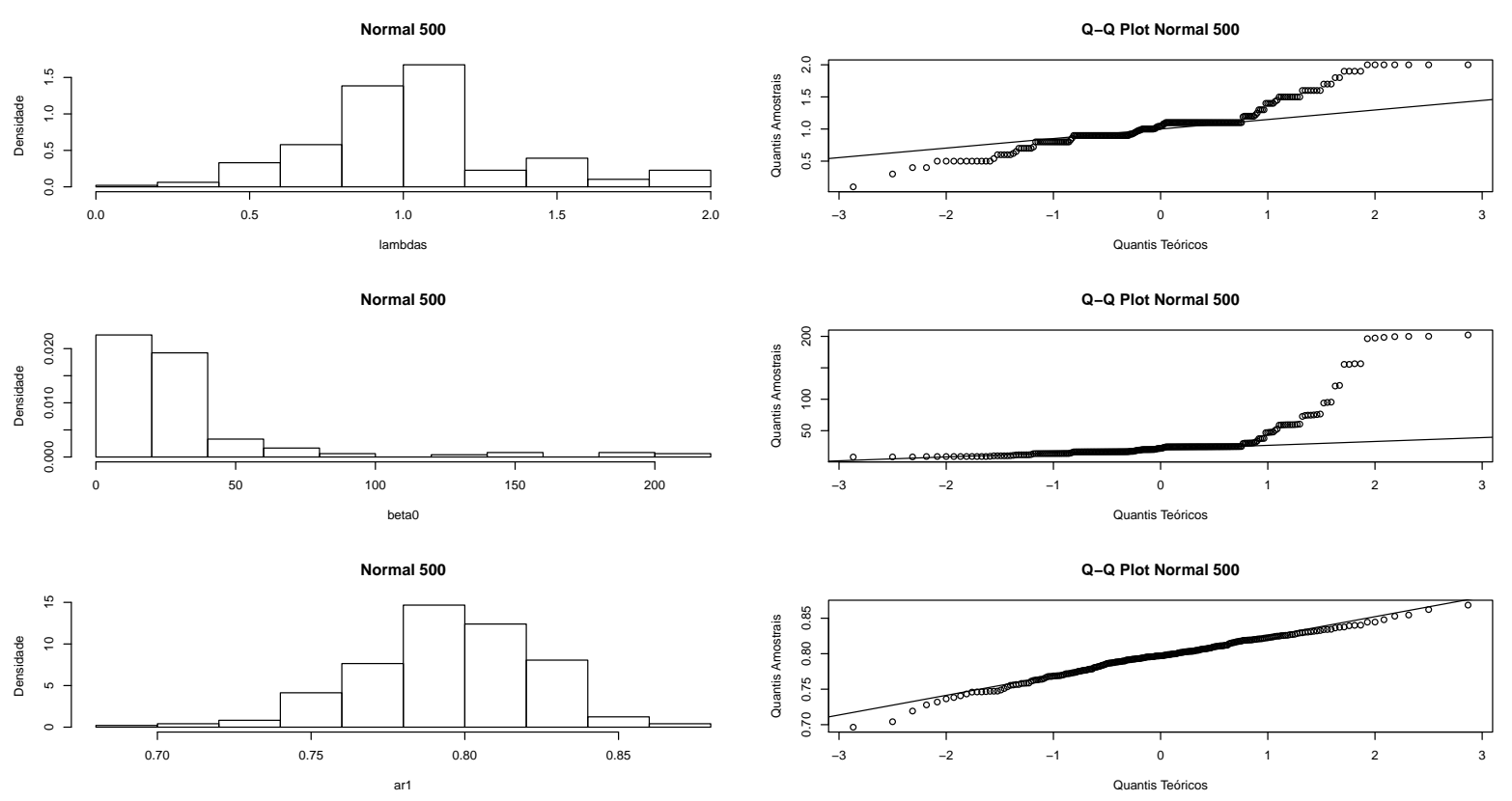

Figura 3.1: Histogramas e QQ-plot para os estimadores de $\lambda, \beta_{0}, \varphi$, respectivamente do modelo normal heteroscedástico com transformação.

Dados foram gerados usando-se um modelo similar ao (3.11), considerando a distribuição t-Student com 30 graus de liberdade. Os resultados para 50 amostras de tamanho $T=500$ 

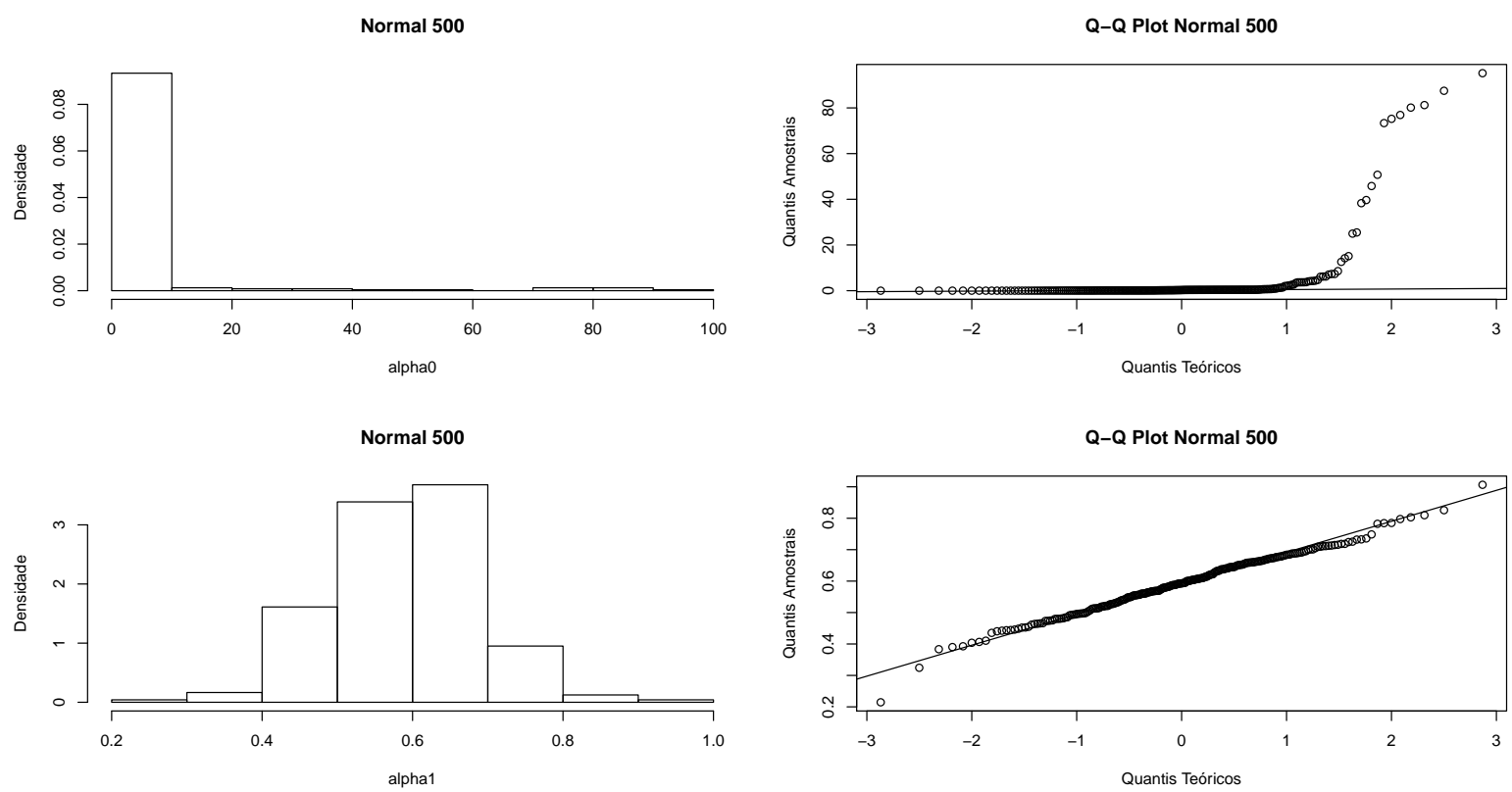

Figura 3.2: Histogramas e QQ-plot para os estimadores de $\alpha_{0}$ e $\alpha_{1}$, respectivamente do modelo heteroscedástico normal com transformação.

encontram-se na Tabela 3.2. Observando os vieses e EQM, podemos ver que estes são menores que os analisados na Tabela 3.1, ou seja o comportamento das estimativas para amostras geradas a partir da distribuição t-Student(30) ficou melhor, no que diz respeito ao viés e EQM. A partir das Figuras 3.3 e 3.4, notamos que o comportamento das estimativas são semelhantes ao modelo com distribuição normal.

\begin{tabular}{crcrcc}
\hline$T$ & $\hat{\lambda}$ & \multicolumn{1}{c}{$\hat{\beta}_{0}$} & $\hat{\varphi}$ & \multicolumn{1}{c}{$\hat{\alpha_{0}}$} & \multicolumn{1}{c}{$\hat{\alpha_{1}}$} \\
\hline & & & & & \\
500 & 1,015 & 24,952 & 0,801 & 0,906 & 0,569 \\
Viés & $-0,015$ & $-4,952$ & $-0,001$ & $-0,706$ & 0,031 \\
EQM & 0,069 & 296,463 & 0,001 & 5,721 & 0,007
\end{tabular}

Tabela 3.2: Valores para os EMV dos respectivos parâmetros $\lambda=1, \beta_{0}=20, \varphi=0,8, \alpha_{0}=0,2$ e $\alpha_{1}=$ 0,6 para distribuição t-Student(30).

\subsection{Aplicação}

Nesta seção, modelamos uma série temporal real. O conjunto de dados utilizados foi a série de preços diários da Petrobras desde 02 de janeiro de 1995 até 27 de dezembro de 2000.

A Figura 3.5 apresenta o gráfico da série de preços diários da Petrobras, conjuntamente com os gráficos da função de autocorrelação e autocorrelação parcial indica que a série apresenta tendência estocástica e volatilidade, sugerindo, assim um modelo heteroscedástico. A 

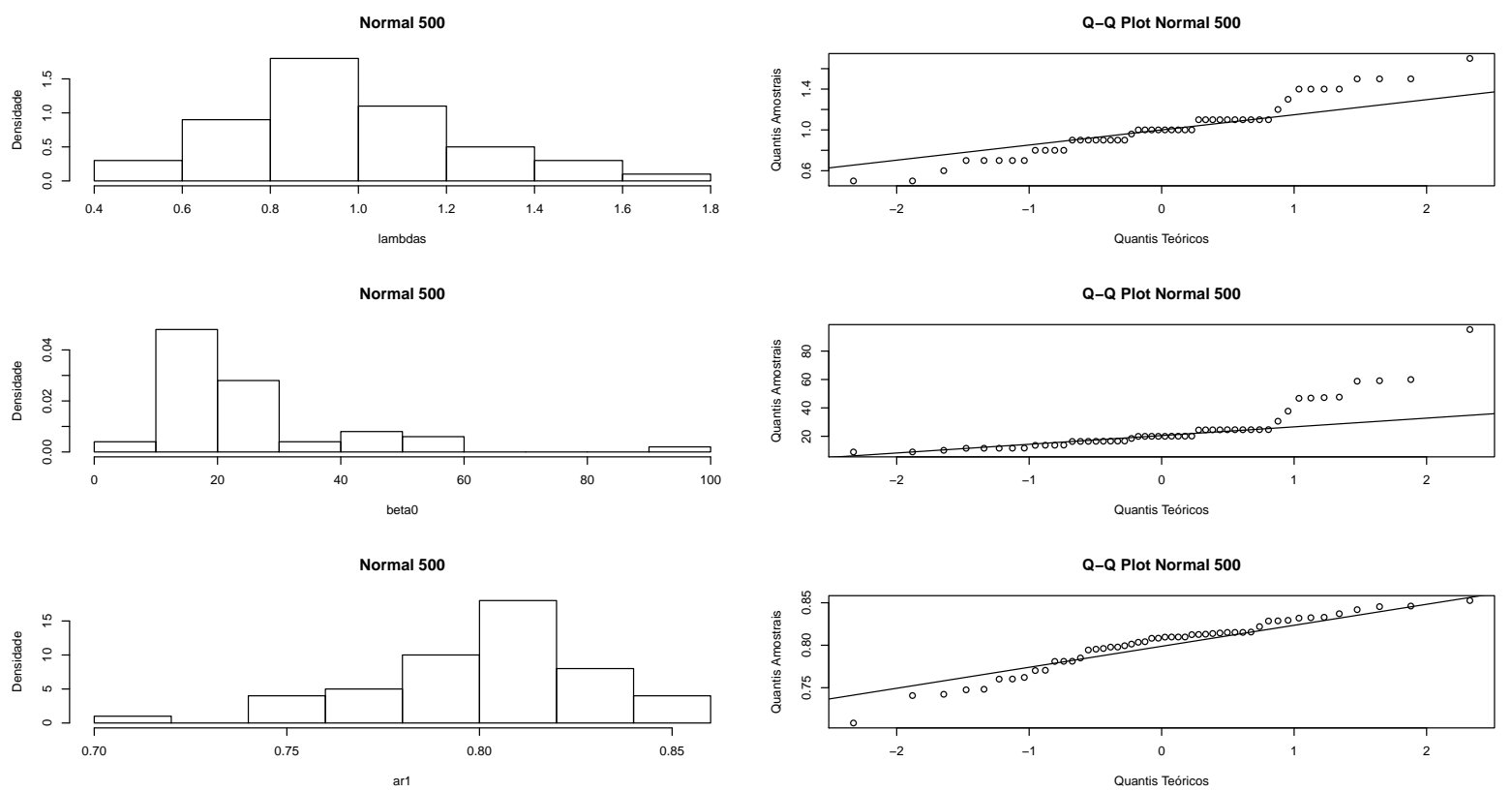

Figura 3.3: Histogramas e QQ-plot para os estimadores de $\lambda, \beta_{0}, \varphi$, respectivamente do modelo heteroscedástico com transformação e distribuição t-Student(30).
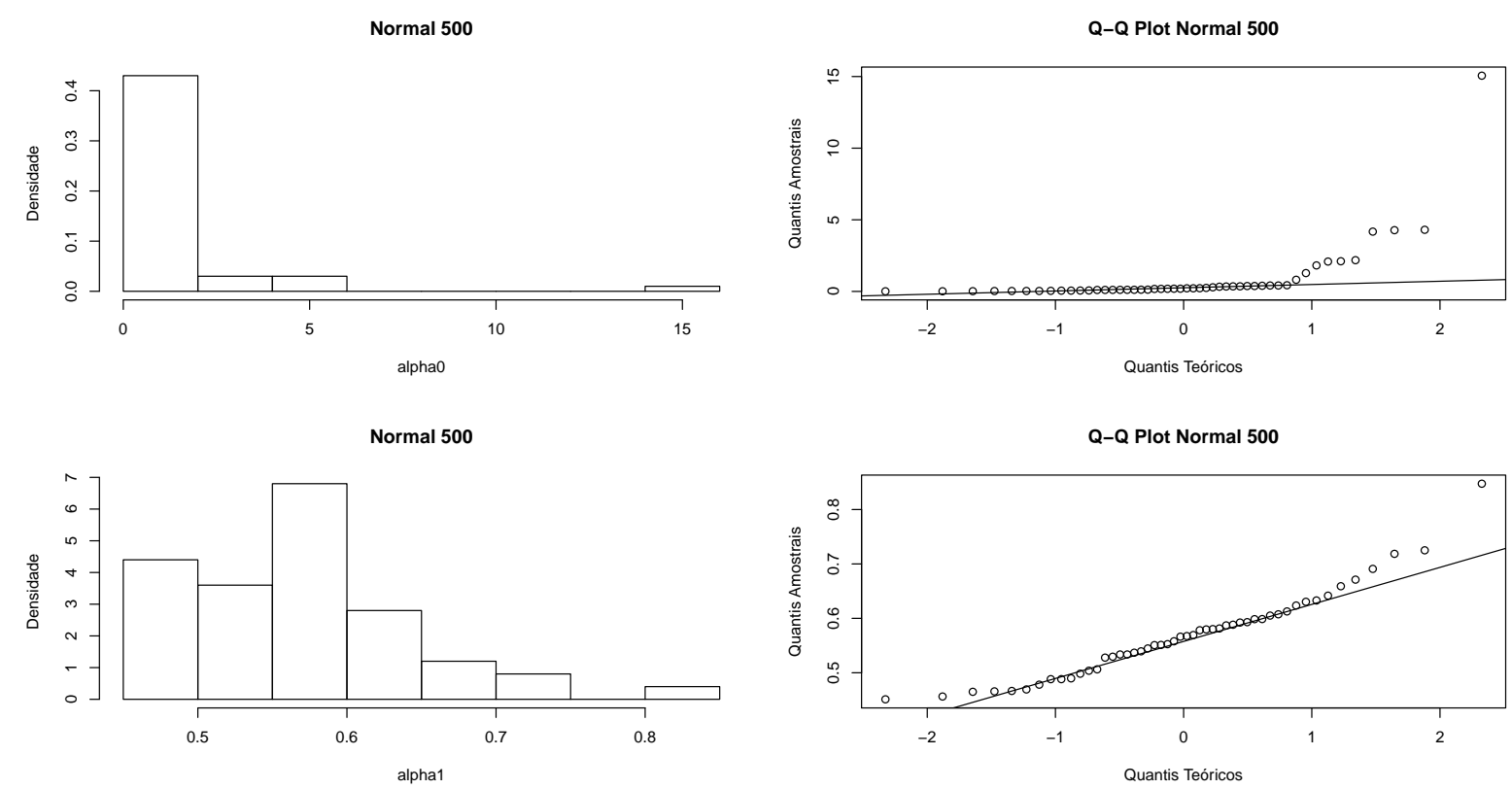

Figura 3.4: Histogramas e QQ-plot para os estimadores de $\alpha_{0}$ e $\alpha_{1}$, respectivamente do modelo heteroscedástico com transformação e distribuição t-Student(30).

fim de investigar a ordem do modelo TSHARMA, inicialmente modelamos a série com modelos TSARMA. Verificamos que o parâmetro de transformação estava muito perto de 0 (zero) e existia um componente autoregressivo de ordem 1, significativo com valor próximo a 1 (um), justificando assim, a transformação logarítmica e diferença que, usualmente, se faz nesse tipo de série temporal. Quando analisamos os gráficos da função de autocorrelação e autocorrelação par- 
cial dos resíduos ao quadrado, Figura 3.6, confirmamos a presença de volatilidade, necessitando assim um modelo da classe TSHARMA. Pelo decaimento lento da função de autocorrelação dos resíduos ao quadrado e o corte na função de autocorrelação parcial dos resíduos ao quadrado, temos indícios que um modelo TSHARMA similar ao $\mathrm{ARCH}(9)$ trataria bem a série. Então, modelamos o logarítmo dos retornos, log-retornos, da série de preços diários da petrobras com um modelo TSHARMA, em que a estrutura de regressão para a média é similar a um AR(5) com intercepto e a estrutura de regressão para a variância é similar ao $\mathrm{ARCH}(9)$ com distribuição normal. Como a série de log-retornos da petrobras é resultado de transformações na série de preços da petrobras, fixamos $\lambda=1$ para facilitar a escolha do modelo TSHARMA.

As estimativas para os parâmetros do modelo ajustado e seus, respectivos, desvios padrões fica dado na Tabela 3.3. A Figura 3.7 apresenta os valores das funções de autocorrelação e autocorrelação parcial para os resíduos e resíduos ao quadrado do modelo TSHARMA ajustado, podemos ver que o modelo TSHARMA se ajustou muito bem a série de log-retornos da petrobras.

\begin{tabular}{lrc}
\hline & estimativa & desvio padrão \\
\hline$\hat{\beta_{0}}$ & 0,0011 & 0,0007 \\
$\hat{\varphi_{1}}$ & 0,1710 & 0,0301 \\
$\hat{\varphi_{5}}$ & $-0,0421$ & 0,0267 \\
$\hat{\alpha_{0}}$ & 0,0003 & 0,00003 \\
$\hat{\alpha_{1}}$ & 0,1677 & 0,0364 \\
$\hat{\alpha_{2}}$ & 0,1598 & 0,0363 \\
$\hat{\alpha_{3}}$ & 0,2014 & 0,0394 \\
$\hat{\alpha_{4}}$ & $-0,0249$ & 0,0096 \\
$\hat{\alpha_{5}}$ & 0,0258 & 0,0202 \\
$\hat{\alpha_{7}}$ & 0,1319 & 0,0329 \\
$\hat{\alpha_{9}}$ & 0,0715 & 0,0263 \\
\hline
\end{tabular}

Tabela 3.3: Valores para as estimativas dos parâmetros para o modelo TSHARMA com distribuição normal. 

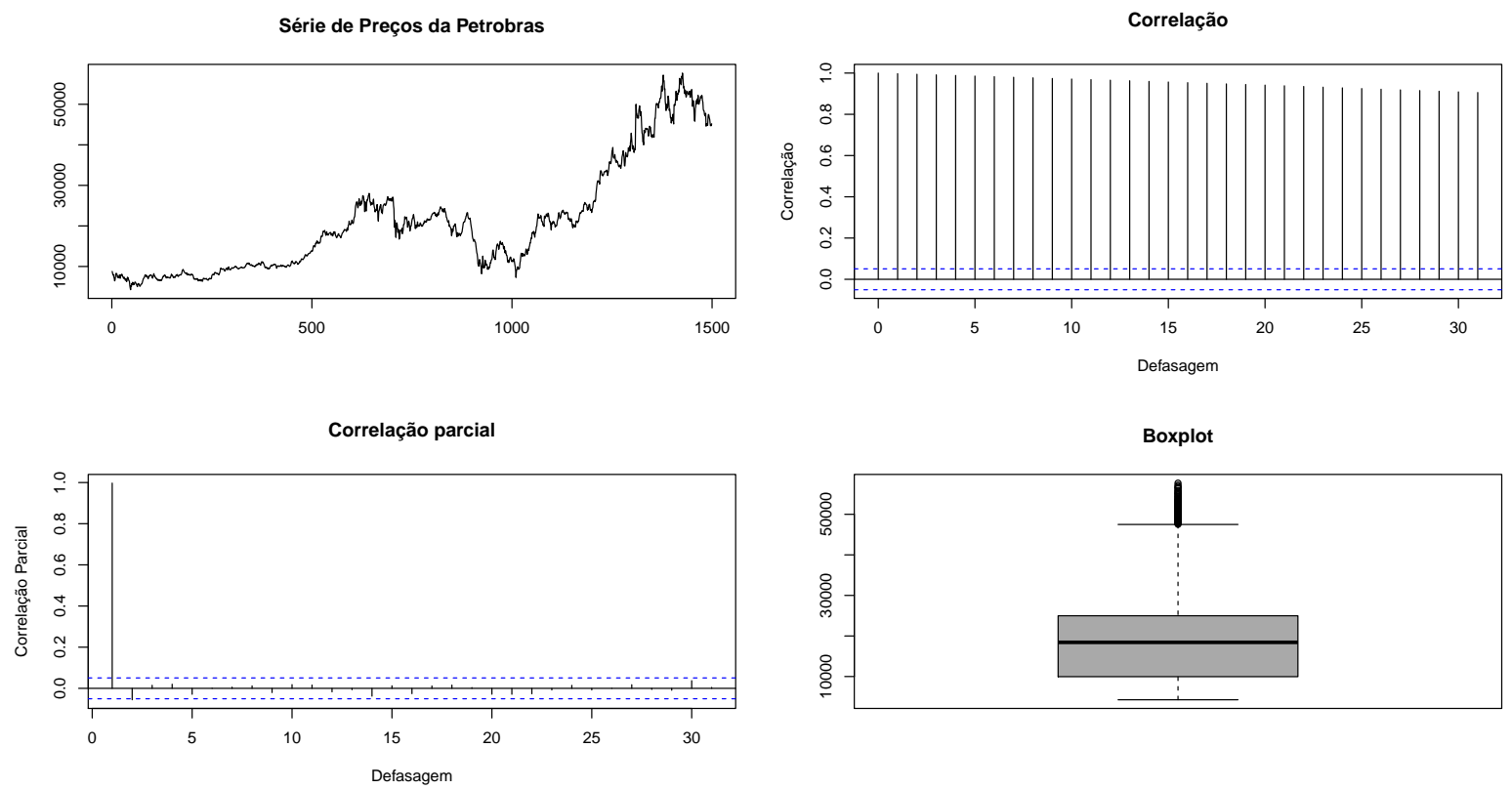

Figura 3.5: Série de preços da Petrobras, função de autocorrelação, função de autocorrelação parcial e box-plot.
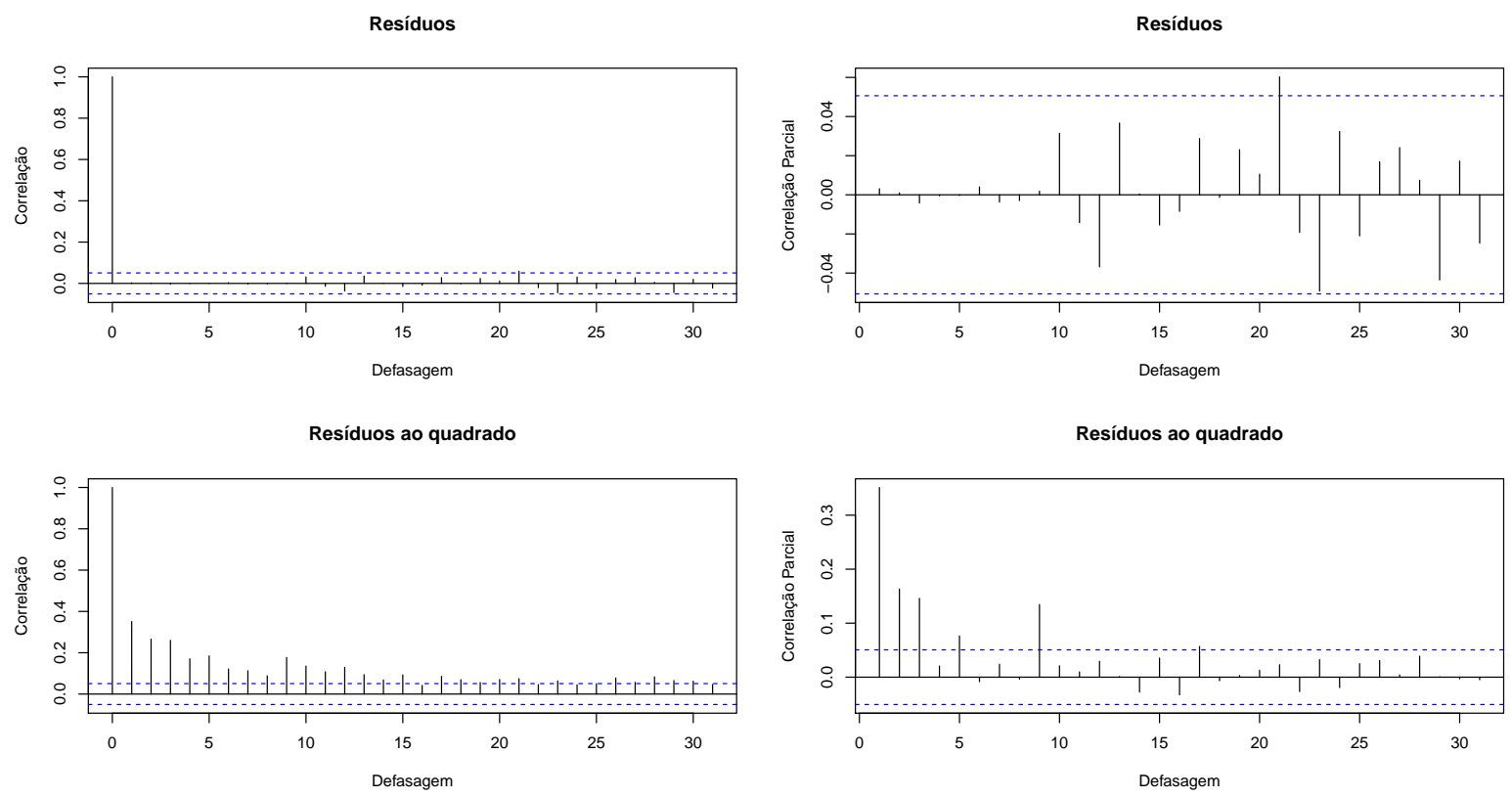

Figura 3.6: Função de autocorrelação, função de autocorrelação parcial dos resíduos e dos resíduos ao quadrado da modelagem TSARMA com distribuição normal. 

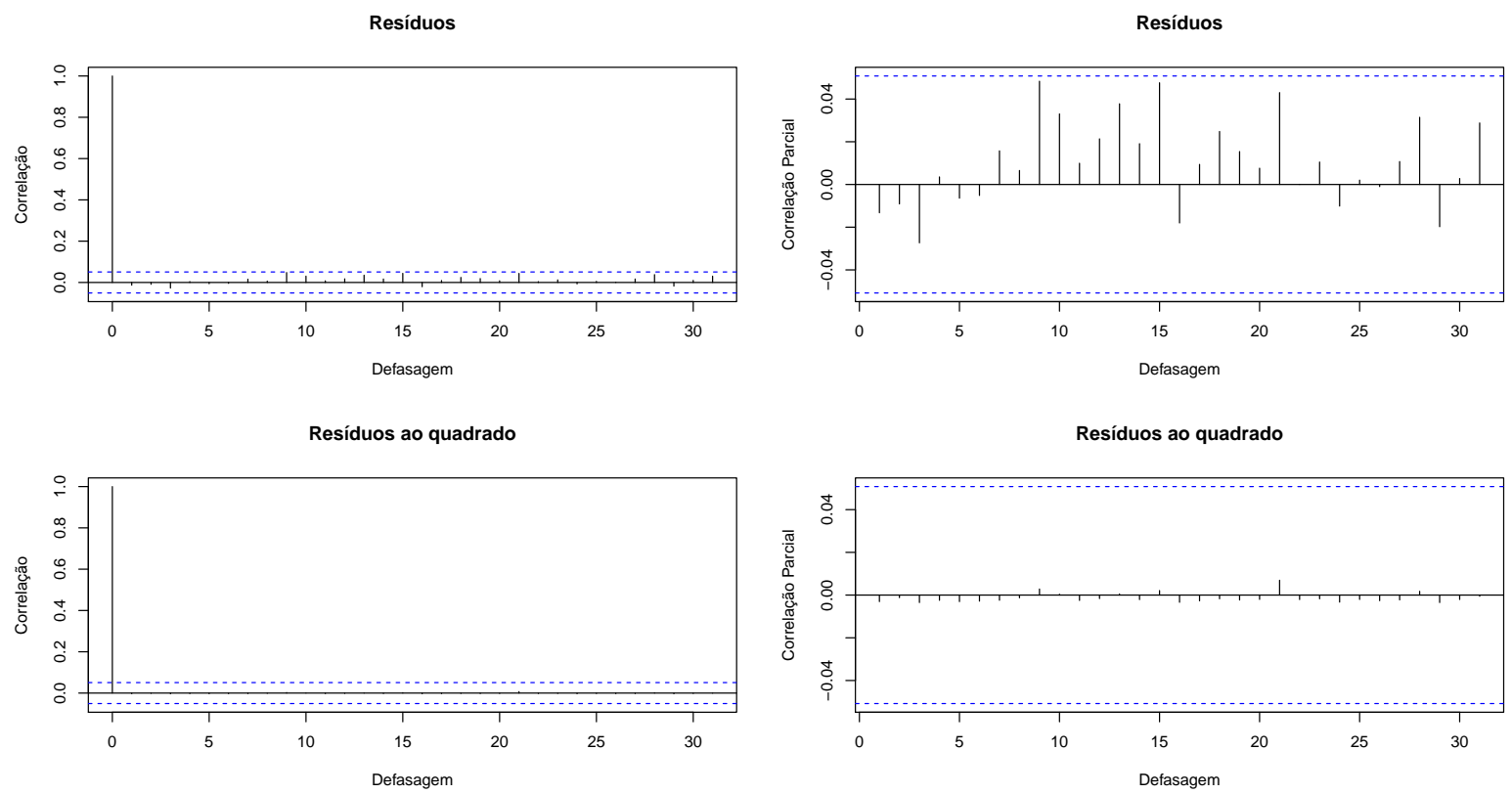

Figura 3.7: Função de autocorrelação, função de autocorrelação parcial dos resíduos e dos resíduos ao quadrado da modelagem TSHARMA com distribuição normal. 


\section{Capítulo 4}

\section{Conclusões e Pesquisas Futuras}

Nesta tese, discutimos vários aspectos envolvendo modelos de regressão simétricos transformados para séries temporais. Quando a suposição de homoscedasticidade é válida, a classe de modelos TSARMA foi proposta, juntamente com um processo iterativo para estimação do parâmetro de transformação, $\lambda$, do vetor paramétrico para a estrutura de regressão para a média, $\nu$ e escala, $\phi$. Verificamos para a classe de modelos TSARMA que a distribuição assintótica dos estimadores dos parâmetros $\nu$ e $\phi$ é normal. Considerando o resíduo com $\phi$ conhecido ou fixo, e seguindo Li (1992) verificamos, também, que a distribuição assintótica da autocorrelação residual amostral é normal. Seguindo a ideia de Cysneiros (2004) que por sua vez se baseou em Cox e Snell (1968) corrigimos os dois primeiros momentos dos resíduos até ordem $n^{-1}$, em que $n$ é o tamanho da amostra.

Outro aspecto abordado foi utilizar um método análogo ao método small- $\theta$ apresentado em Draper e Cox (1969) para obter estimativas da série na sua escala original considerando a transformação de Box-Cox.

Estudos de simulações mostraram que pequenas variações das estimativas do parâmetro de transformação em relação ao seu valor verdadeiro, $\lambda=1$, conduzem a grandes variações nas estimativas de alguns parâmetros do modelo em relação aos seus respectivos valores verdadeiros, pois causa diferença na grandeza dos dados e, necessariamente, as estimativas do intercepto da estrutura de regressão para a média fica muito longe do seu valor verdadeiro, assim como os parâmetros de médias móveis que também dependem da escala da série. Uma aplicação com dados reais foi realizada. Comparamos a modelagem usual de Box-Jenkins com a proposta, TSARMA, e mediante algumas medidas, observamos que um modelo TSARMA superou a modelagem usual.

Quando a suposição de homoscedasticidade não é verificada, modelos de regressão simétricos 
transformados heteroscedásticos, TSHARMA, foram propostos. Desenvolvemos um processo iterativo para a estimação dos parâmetros de locação e escala. Vimos que, o modelo TSHARMA é uma extensão natural da classe de modelos TSARMA proposta no Capítulo 2, que por sua vez, é uma extensão da classe de modelos GARMA desenvolvida por Benjamin et al. (2003) e da classe de modelos TGARMA proposta por Cordeiro e Andrade (2009).

A fim de estudar o comportamento das estimativas para a classe de modelos TSHARMA, realizamos algumas simulações que mostraram que a estimativa do parâmetro de transformação interfere nos resultados das estimativas para os outros parâmetros do modelo, assim como na classe de modelos TSARMA. Além de interferir em alguns estimadores da estrutura de regressão para a média, também notamos que a estimativa do parâmetro de transformação interfere em alguns estimadores da estrutura de regressão para a variância. Realizamos uma aplicação com a série temporal de preços diários da Petrobras. Constatamos que a modelagem TSHARMA se torna muito lenta se o modelo possui muitos parâmetros para serem estimados, contudo funciona bem e modelou a série satisfatoriamente.

Vários trabalhos poderão ser desenvolvidos a partir das ideias desta tese. Dentre estes, podemos citar:

- Mediante alguns problemas encontrados, tem surgido como uma alternativa utilizar o filtro de Kalman, ou seja, representar o modelo TSARMA na forma espaço-estado e então utilizar o filtro de Kalman como processo recursivo de estimação. O filtro de Kalman é um conjunto de equações matemáticas que constitui um processo recursivo que permite a estimação dos parâmetros desconhecidos do modelo por meio da maximização da verossimilhança via decomposição do erro de previsão.

- Dar continuidade aos estudos acerca dos modelos TSHARMA. Como por exemplo, estudar o teste da razão de verossimilhanças, assim como caracterizar a distribuição assintótica dos estimadores dos parâmetros.

- Estender a metodologia de Benjamin et al. (2003) e Cordeiro e Andrade (2009) para modelos ARIMA com memória longa, por exemplo os modelos ARFIMA.

- Propor uma classe de modelos transformados GTR ("generalized threshold regression"). Samia et. al. (2007) consideram o modelo GTR para regressão linear com dados de séries temporais não normais. O objetivo será considerar o modelo TGTR (transformed generalized 
theshold regression) e estudar, um caso particular de grande utilidade em séries temporais, o modelo TAR ("threshold autoregressive") de Tong (1983).

- Utilizar a inferência indireta, proposta em Gouriéroux et. al. (1993), como alternativa para a estimação dos parâmetros dos modelos TSHARMA. 



\section{Referências Bibliográficas}

Akaike, H. (1973). Information theory and an extension of the maximum likelihood principle. In B.N. Petrov and F. Csaki, eds.. Second International Symposium on Information Theory, 267-281, Budapest, Akademiai Kaido. 5

Akaike, H. (1974). A new look at the statistical model identification. IEEE Transactions on automatic control,AC-19, 716-723. 7

Albert, J., Delampady, M. and Polasek, W., (1991). A class of distributions for robustness studies. J. Statist. Planning Inference, 28, 291-304. 4

Benjamin, M.A., Rigby, R.A. and Stasinopoulos, D.M. (2003). Generalized autoregressive moving average models. J. Amer. Statist. Assoc., 98, 214-223. iii, v, 1, 52

Blake, I. F. and Thomas, J. B. (1968). On class of processes arising in linear estimation theory. IEEE. Trans. Info. Theory, 14, 12-16.

Bollerslev, T. (1986). Generalized autoregressive conditional heteroskedasticity. Journal of Econometrics, 31, 307-327.

Bollerslev, T. (1987). A conditionally heterokedastic time series model for speculative process and rates of return. Review of Economics and Statistics, 69, 542-547.

Bollerslev, T. (1988). On the correlation structure for the generalized autoregressive conditional heteroskedasticity. Journal of Time Series Analysis, 9, 121-132.

Box, G.E.P. and Cox, D.R. (1964). An analysis of transformation. Journal of the Royal Statistical Society, Ser. B, 26, 211-252. 4

Box, G.E.P. and Pierce, D.A. (1970). Distribution of the residual autocorrelations in autoregressive integrated moving average time series models. J. Amer. Statist. Assoc., 65, 1509-1526. 15 
Box, G. E. P. and Tiao, G. C. (1973). Bayesian Inference in Statistical Analysis. Addison-Wesley, London. 3

Cambanis, S., Huang, S. and Simons, G. (1981). On the theory of elliptically contoured distributions. J. Multivariate Anal., 11, 368-385.

Chmielewski, M. A. (1981). Elliptically symmetric distributions: a review and bibliography. Internat. Statist. Rev., 49, 67-74.

Cordeiro, G. M. and de Andrade, M. G. (2009). Transformed generalized linear models. J. Statist. Planning Inference, 139, 2970-2987. iii, 1, 19, 52

Cox, D.R. (1961). Tests of separate families of hypotheses. In Proceedings of the Fourth Berkeley Symposium on Mathematical Statistics and Probability, 105-123. Berkeley, University of California Press. 6

Cox, D.R. (1962a). Further results on tests of separate families of hypotheses. Journal of the Royal Statistical Society, Ser. B, 24, 406-424. 6

Cox, D. R. and Hinkley, D. V. (1974). Theoretical Statistics. London: Chapman and Hall. 10

Cox, D.R. and Snell, E.J. (1968). A general definition of residuals. Journal of the Royal Statistical Society, Ser. B, 30, 248-275. 17, 51

Cysneiros, F.J.A. (2004). Métodos restritos e validação de modelos simétricos de regressão. Tese de doutorado, Departamento de Estatística, Universidade de São Paulo, Brasil. 17, 18, 51

Cysneiros, F. J. A. and Paula, G. A. (2005). Restricted methods in symmetrical linear regression models. Comput. Statist. Data Analysis, 49, 689-708. 13

Davies, Triggs e Newbold (1977). Significance of the Box-Pierce Portmanteau Statistics in Finite Samples. Biometrika 64, 517-522. 16

Ding, Z., Granger, C.W.J. and Engle, R.F. (1993). A long memory property of stock returns and a new model. Journal of Empirical Finance, 1, 83-106.

Draper, N. R. and Cox, D. R. (1969). On distributions and their transformation to normality. Journal of the Royal Statistical Society, Ser. B, 31, 472-476. 19, 51 
Engle, R. F. (1982). Autorregressive conditional heteroskedasticity with estimates of the variance of U. K. inflation. Econometrica, 50, 987-1008.

Fahrmeir, L. and Kaufmann, H. (1985). Consistency and asymptotic normality of the maximum likelihood estimator in generalized linear models. The Annals of Statistics, 13, 342-368. 14

Feigin, P.D. (1981). Condicional exponential families and representation theorem for asymptotic inference. The Annals of Statistics, 24, 295-320. 1

Galea, M., Paula, G.A. and Cysneiros, F.J.A. (2005). On diagnostics in symmetrical nonlinear models. Statistics 63 Probability Letters, 73, 459467.

Glosten, L.R., Jagannathan, R. and Runkle, D. (1993). Relationship between the expected value and the volatility of the nominal excess return on stocks. Journal of Finance, 48, 1779-1801.

Gouriéroux, C., A. Monfort and E. Renault (1993). Indirect inference. Journal of Applied Econometrics, 8, 85-118. 53

Granger, C. W. J. and Newbold, P. (1986). Forecasting Economic Time Series, 2nd edn, New York: Academic Press. 18

Green, P. (1984). Iterative reweighted least squares for maximum likelihood estimation, and some robust and resistant alternatives (with discussion). Journal of the Royal Statistical Society, Ser. B, 46, 149-192. 14

Grunwald, G. K., Hyndman, R. J., Tedesco, L. M. and Tweedie, R. L. (2000). Non-Gaussian conditional linear AR(1) models. Australian and New Zealand Journal of Statistics, 42, 179495. 1

Guerrero, V. M. (1993). Time-series analysis supported by power transformations. Journal of Forecasting, 12, 37-48. 18, 19

Heyde, C. C. (1978). On an optional asymptotic property of the maximum likelihood estimator of a parameter from a stochastic process. Stochastic Processes and Their Applications, 8, 1-9. 1

Heyde, C. C. and Feigin, P. D. (1975). On Efficiency and Exponential Families in Stochastic Process Estimation. In Statistical Distributions in Scientific Work, Vol. 1, eds. G. P. Patil et al., Dordrecht: D. Riedel, 227-240. 1 
Kaufmann, H. (1987). Regression models for nonstationary categorical time series: Asymptotic estimation theory. Annals of Statistics, 15, 79-98. 15

Kelker, D. (1970). Distribution theory of spherical distributions and a location-scale parameter generalization. Sankhyã , 32, 419-430. 4

Kotz, S. (1975). Multivariate distributions at a cross-road. In: Patil G. P., Kotz, S., Ord, J. K., (Eds.), Statistical Distributions in Scientific Work, 1, pp. 247-270. 3

Kullback, S., and R. A. Leibler (1951). On information and sufficiency. Annals of Mathematical Statistics, 22, 79-86. 5, 7

Lange, K.L.; Little, R.J.A. and Taylor, J.M.G. (1989). Robust statistical modeling using the $t$ distribution. Journal of the American Statistical Association, 84, 881-896. 12

Li, W.K. (1992). On the asymptotic distribution of residual autocorrelations in nonlinear time series modelling. Biometrika, 79, 435-437. 16, 51

Li, W. K. (1994). Time series models based on generalized linear models: Some further results. Biometrics, 50, 506-511. 1

Ljung, G.M. and Box, G.E.P. (1978). On a measure of lack of fit in time series models. Biometrika, 65, 297-303. 16

Manly, B. F. (1976). Exponential data transformation. The Statistician, 25, 37-42. 4

McGraw, D. K. and Wagner, J. F. (1968). Elliptically symmetric distributions. IEEE. Trans. Inform. Theory, 14, 110-120.

Miller, D. M. (1984). Reducing transformation bias in curve fitting. The American Statistician, 38, 124-126. 18

Nelson, D.B. (1991). Conditional heteroskedasticity in asset returns. Econometrica, 59, 347-370.

Nelson, D.B. and Cao, C.Q. (1992). Inequality constraints in the univariate GARCH model. Journal of Business and Economic Statistics, 10, 229-235.

Neyman, J. and Scott, E. L. (1960). Correction for bias introduced by a transformation of variables. Annals of Mathematical Statistics, 31, 643-655. 18 
Pankratz, A. and Dudley, U. (1987). Forecasts of power-transformed series. Journal of Forecasting, 6, 239-248. 18, 19

Pesaran, M.H. (1987). Global and partial non-nested hypotheses and asymptotic local power. Econometric Theory, 3, 677-694. 5

Sakia, R.M. (1992). The Box-Cox transformation technique: a review. The Statistician, 41, 168-178. 4

Samia, N. I., Chan, K. S. and Chr.Stenseth, N. (2007). A generalized threshold mixed model for analyzing non-normal nonlinear time series: with application to plague in Kazakhstan. Biometrika, 94, 101-118. 52

Schwarz, G. (1978). Estimating the dimension of a model. Annals of Statistics, 6, 461-464. 6

Serfling, R. J. (1980). Approximation Theorems of Mathematical Statistics. New York: John Wiley. 10

Taylor, J. M. G. (1986). The retransformed mean after a fitted power transformation. J. Amer. Statist. Assoc., 81, 114-118. 18, 19

Tong, H. (1983). Threshold Models in Nonlinear Time Series Analysis. Lecture Notes in Statistics, 21, Heidelberg: Springer. 53

Vuong, Q.H. (1989). Likelihood ratio tests for model selection and non-nested hypothesis. Econometrica, 57, 307-333. 5, 7

Zakoian, J.M. (1994). Threshold heteroskedasticity models. Journal of Economic Dynamics and Control, 18, 931-955.

Zeger, S. L., and Qaqish, B. (1988). Markov Regression Models for Time Series: A QuasiLikelihood Approach. Biometrics, 44, 1019-1032. 1 